\title{
Arctic Ocean Tides from GRACE Satellite Accelerations
}

\author{
by \\ Bryan Killett
}

B.S., University of Oregon, 2004

M.S., University of Colorado, 2007

\author{
A thesis submitted to the \\ Faculty of the Graduate School of the \\ University of Colorado in partial fulfillment \\ of the requirements for the degree of \\ Doctor of Philosophy \\ Department of Physics
}

2011 
This thesis entitled:

Arctic Ocean Tides from GRACE Satellite Accelerations

written by Bryan Killett

has been approved for the Department of Physics

John Wahr

Neil Ashby

Peter Bender

Robert Leben

Steve Nerem

Date

The final copy of this thesis has been examined by the signatories, and we find that both the content and the form meet acceptable presentation standards of scholarly work in the above mentioned discipline. 
Killett, Bryan (Ph.D., Physics)

Arctic Ocean Tides from GRACE Satellite Accelerations

Thesis directed by Prof. John Wahr

Models are routinely used to remove the effects of global ocean tides from GRACE data during processing to reduce temporal aliasing into monthly GRACE solutions. These models have typically been derived using data from satellite altimeter missions such as TOPEX/Poseidon. Therefore the Arctic ocean components of tide models aren't constrained by altimetry data, potentially resulting in errors that are likely to alias into monthly GRACE gravity fields at all latitudes.

Seven years of GRACE inter-satellite accelerations are inverted to solve for corrections to the amplitude and phase of major solar and lunar ocean tides at latitudes north of $50^{\circ} \mathrm{N}$ using a mascon approach. The tide model originally applied to our data was FES2004, truncated to maximum degree $l_{\max }=90$. Simulations are performed to verify that our inversion algorithm works as designed. Uncertainty estimates are derived from tidal solutions on land, and by subtracting two independent solutions that each use 3.5 years of data. Features in the $M_{2}$ and $K_{1}$ solutions that rise above the noise floor likely represent errors in the FES2004 model. Errors due to truncating the spherical harmonic expansion of FES2004 are too small, and errors in the land mask model (needed to transform sea surface heights into mass) only affect coastal areas and don't produce similar relative amplitudes in both tidal constituents. In the oceans north of $50^{\circ} \mathrm{N}$, these residual estimates tend to reduce the FES2004 amplitudes for $M_{2}, K_{1}, O_{1}$, and $P_{1}$.

The power spectra of accelerations are analyzed, and reductions in the variance of accelerations not used in our inversion suggest that our results can be used to improve GRACE processing. 


\section{Dedication}

To my parents, who always encouraged me; and my grandmothers, who taught me the values of humility and forthrightness. To the memories of my grandfathers, who (literally) taught me to fly; and Aunt K, my own personal scientific patron. 


\section{Acknowledgements}

This research wouldn't have been possible without John Wahr's legendary patience and seemingly clairvoyant ability to guide me away from conceptual dead ends. I'll probably spend the rest of my life trying to live up to his example of "ego-less science." Shailen Desai, Dah-Ning Yuan, and Michael Watkins made me welcome at JPL, helped introduce me to the nuances of ocean tides and GRACE data analysis, and provided valuable feedback on the paper we co-authored regarding this research. I'm grateful to Neil Ashby, Peter Bender, Robert Leben, and Steve Nerem for being on my committee, and for their many helpful comments.

Thanks to the FES2004 authors for their model and sharing the code; Wenwen Lu and Sung Byun for providing the GRACE acceleration data; Abbas Khan, Cecilia PeraltaFerriz, Jamie Morison and Andrey Proshutinsky for providing tide gauge data; Fan-Chi Lin for suggestions that led to the zone of influence algorithm; Richard Ray for insight into the origin of the north pole anomaly in $K_{1}$; Jenni Bonin for correctly insisting that the degree 90 truncation errors of FES2004 are below the GRACE noise floor; Richard Eanes for

reminding me why Greenwich phase lags are physically meaningful; Lin Liu, Xinxing Wang and Wouter van der Wal for many useful discussions and for sharing code; Sean Swenson for writing the first version of the IDL graphics code; and Junseong Heo and Mikhail Barmin for their superb technical assistance. This work is supported by NASA grant NNX08AF026 and JPL contract 1390432 to the University of Colorado. 


\section{Contents}

1 Introduction $\quad 1$

1.1 The Newtonian Gravitational Potential . . . . . . . . . . . . . . . . 3

1.2 The Gravitational Tidal Potential . . . . . . . . . . . . . . . . . 7

1.3 Harmonic Analysis of the Tidal Potential . . . . . . . . . . . . . . . . 10

2 GRACE $\quad 22$

3 Methodology 25

3.1 Zone of Influence . . . . . . . . . . . . . . . . . . . . . . 28

3.2 Support Grid Points . . . . . . . . . . . . . . . . . . 30

3.3 Overlapping Regions . . . . . . . . . . . . . . . . . . . 32

4 Simulations $\quad 36$

5 Real Data Inversions $\quad 40$

5.1 Uncertainty Estimates . . . . . . . . . . . . . . . . . . 47

5.2 Noise Reduction . . . . . . . . . . . . . . . . . . . . 51

5.3 Power Spectra . . . . . . . . . . . . . . . . . . . 52

5.4 The $K_{1}$ North Pole Anomaly . . . . . . . . . . . . . . . . . . . . . . . . 55

5.5 Comparisons with Tide Gauge Data . . . . . . . . . . . . . . 56

6 Conclusions $\quad 62$ 
A Spherical Harmonics 


\section{Tables}

1.1 Astronomical frequencies and phase angles used to define the tidal potential. 15

1.2 Tidal frequencies and amplitudes for tides in the Cartwright-Tayler-Edden

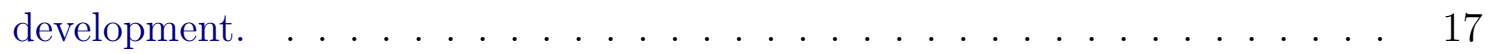

1.3 Fundamental astronomical periods. . . . . . . . . . . . . . . . . 20

2.1 Tidal alias periods in GRACE data. . . . . . . . . . . . . . . . 24

5.1 Variance reduction due to tides. . . . . . . . . . . . . . . 52

5.2 AutoFES versus in situ tide gauge data at Yakutat, AK . . . . . . . . . . 59

5.3 MasconFES versus autoFES at Yakutat, AK . . . . . . . . . . . . 60

5.4 Inverted real data versus in situ tide gauge minus autoFES at Yakutat, AK . 61

A.1 Associated Legendre functions. . . . . . . . . . . . . . . . . . 72

A.2 Spherical harmonics $Y_{l}^{m}(\theta, \phi)$ for $1=0,1,2 \ldots \ldots \ldots \ldots$ 


\section{Figures}

1.1 Total gravitational forces and tidal forces due to the Moon. . . . . . . . . . 1

1.2 Two geocentric coordinate systems. . . . . . . . . . . . . . . . . . 4

1.3 A geocentric Earth-fixed coordinate system. . . . . . . . . . . . . 8

1.4 The lunar node. . . . . . . . . . . . . . . . . . . . . 10

1.5 Reference points projected onto the ecliptic plane. . . . . . . . . . . . 12

1.6 Angles measured in the equatorial plane. . . . . . . . . . . . . . . . . 12

1.7 Diurnal and semidiurnal tidal spectra in the Cartright-Tayler-Edden develop-

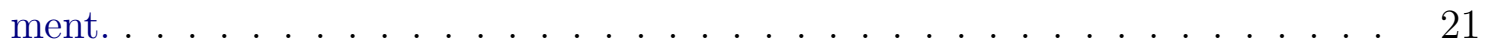

2.1 Simulated GRACE accelerations due to a single mascon. . . . . . . . . . . 23

3.1 Simulation that tests the zone of influence algorithm . . . . . . . . . . 29

3.2 Support grid points. . . . . . . . . . . . . . . . . . . 31

3.3 Simulation that tests the support grid points algorithm. . . . . . . . . . 33

3.4 Overlapping regions, regions-induced error . . . . . . . . . . . . . 35

4.1 Inputs and outputs for two separate simulations. . . . . . . . . . . . . . 37

4.2 Simulation to test resolution. . . . . . . . . . . . . . . . . . . . 39

5.1 Inverted non-tidal parameters: constant, trend, semi-annual, annual. . . . . . 42

5.2 Real data inversions for $M_{2}$ and $K_{1} \ldots \ldots \ldots$. . . . . . . . . . . . 43

5.3 Real data inversions for $O_{1}$ and $P_{1} \ldots \ldots \ldots$. . . . . . . . . . . . . . 44

5.4 Real data inversions for $Q_{1}$ and $N_{2} \ldots \ldots \ldots$. . . . . . . . 45 
5.5 Degree 90 truncation errors for FES2004 $M_{2}$ and $K_{1}$. . . . . . . . . . 48

5.6 Real data inversions for $M_{f}$ and $M_{m} \ldots \ldots \ldots$. . . . . . . . 49

5.7 Power spectra. . . . . . . . . . . . . . . . . . . . . 54

5.8 Investigation of the $K_{1}$ north pole anomaly. . . . . . . . . . . . . . . . 57

A.1 Real parts of the spherical harmonics for $l=0,1,2 \ldots \ldots . \ldots 76$ 


\section{Chapter 1}

\section{Introduction}

Tides are caused by the gravitational forces of the Moon, Sun, and (to a much smaller extent) the other planets (Hartmann and Wenzel 1995). More specifically, tides are caused by variations in these gravitational forces over the surface of the Earth; the average gravitational force is only responsible for Earth's orbital motion. For instance, the point on the Earth directly underneath the Moon (point $A$ in figure 1.1) experiences a stronger gravitational force toward the Moon than point $B$ due to the inverse square nature of gravity. Similarly, points on the Earth that aren't on the line connecting the centers of the Earth and Moon experience a component of the gravitational force towards that line due to the central force nature of gravity.

Ocean tides at coastal cities have been studied for over a century using tide gauge data (Darwin 1883; Doodson 1921). These observed tides are actually the differences between the ocean tides and the solid Earth's body tides. These differences exist because the solid Earth

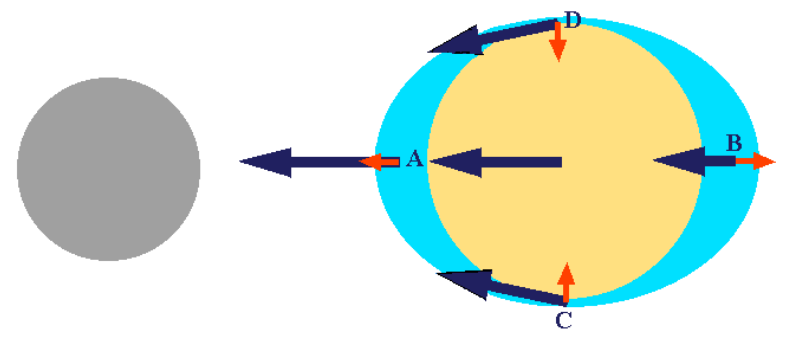

Figure 1.1: Total gravitational forces (blue) and tidal forces (orange) due to the Moon. Adapted from Wikipedia. 
responds nearly instantaneously to the applied tidal forcing, so body tides are in phase with the tidal forcings and have identical spatial patterns (Munk and Macdonald 1975; Wahr 1981; Melchior 1983; Lambeck 1988). In contrast, ocean tides are more complex due to the irregular drag and resonances caused by bathymetry and coastlines interacting with effects of the Earth's rotation. Ocean tides aren't generally in phase with the tidal forcings, and the spatial patterns of ocean tides can vary significantly over short distances, especially in shallow water. One cause of this complexity is that ocean tides dissipate $\sim 2.4 \mathrm{TW}^{1}$ through friction with the seafloor and turbulence, while body tides only dissipate $\sim 0.1 \mathrm{TW}$ (Munk 1997). This friction allows the Earth's rotation to drag the tidal bulge of the ocean so it's not directly under the Moon. That asymmetry exerts a torque on the Moon and the Earth, which is lengthening Earth's day by $\sim 2 \mathrm{~ms}$ per century and causing the Moon to recede from the Earth at a rate of $\sim 3.8 \mathrm{~cm}$ per year (Lambeck 1975; Stephenson et al. 1984; Dickey et al. 1994).

In the 1980s, global ocean tide models (Schwiderski 1980) assimilated tide gauge data to help constrain solutions of tidal hydrodynamic equations. Modern ocean tide models such as FES2004 (Lyard et al. 2006) also rely on satellite altimetry missions such as TOPEX/Poseidon to provide data in deep water, but such data are scarce north of $66^{\circ} \mathrm{N}$. Therefore the Arctic ocean components of the tide models aren't constrained by altimetry data, potentially resulting in errors that could be detected by satellite missions such as GRACE.

\footnotetext{
1 These values are for the lunar $M_{2}$ tide alone. The (current) total dissipation rate for all lunar and solar tides is $~ 3.7$ TW (Munk and Wunsch 1998)
} 


\subsection{The Newtonian Gravitational Potential}

According to Newton's Law of universal gravitation, a point mass at coordinates $\overrightarrow{\mathbf{r}^{\prime}}$ with mass M' exerts a force per unit mass at coordinates $\overrightarrow{\mathbf{r}}$

$$
\overrightarrow{\mathbf{f}}=\frac{G M^{\prime}\left(\overrightarrow{\mathbf{r}^{\prime}}-\overrightarrow{\mathbf{r}}\right)}{\left|\overrightarrow{\mathbf{r}^{\prime}}-\overrightarrow{\mathbf{r}}\right|^{3}}
$$

The associated gravitational potential $V$ is conventionally defined as the negative potential energy per unit mass of this vector field, where $\nabla$ represents the gradient with respect to the coordinates $\overrightarrow{\mathbf{r}}$

$$
\begin{gathered}
\overrightarrow{\mathbf{f}}=\nabla V \\
V=\frac{G M^{\prime}}{\left|\overrightarrow{\mathbf{r}^{\prime}}-\overrightarrow{\mathbf{r}}\right|} .
\end{gathered}
$$

Geocentric coordinate systems such as those in figure 1.2 are convenient. The Cartesian basis vectors $(\hat{\mathbf{x}}, \hat{\mathbf{y}}, \hat{\mathbf{z}})$ are chosen so the point mass M' lies on the $\hat{\mathbf{z}}$ axis. The spherical basis vectors $\left(\hat{\mathbf{e}_{\mathbf{r}}}, \hat{\mathbf{e}_{\theta}}, \hat{\mathbf{e}_{\phi}}\right)$ are oriented differently for each point $\overrightarrow{\mathbf{r}}$, and can be related to the Cartesian basis vectors using equation (1.4).

$$
\left[\begin{array}{c}
\hat{\mathbf{e}_{\mathbf{r}}} \\
\hat{\mathbf{e}_{\theta}} \\
\hat{\mathbf{e}_{\phi}}
\end{array}\right]=\left[\begin{array}{ccc}
\sin \theta \cos \phi & \sin \theta \sin \phi & \cos \theta \\
\cos \theta \cos \phi & \cos \theta \sin \phi & -\sin \theta \\
-\sin \phi & \cos \phi & 0
\end{array}\right]\left[\begin{array}{c}
\hat{\mathbf{x}} \\
\hat{\mathbf{y}} \\
\hat{\mathbf{z}}
\end{array}\right]
$$

This potential can be expanded (see equation A.22) as an infinite series of spherical harmonics by defining $r^{\prime} \equiv\left|\overrightarrow{\mathbf{r}^{\prime}}\right|=r_{>}$and $r \equiv|\overrightarrow{\mathbf{r}}|=r_{<}$, which applies when the mass M' is farther away from the center of the Earth than the point $\overrightarrow{\mathbf{r}}$ at which $V$ is measured. 


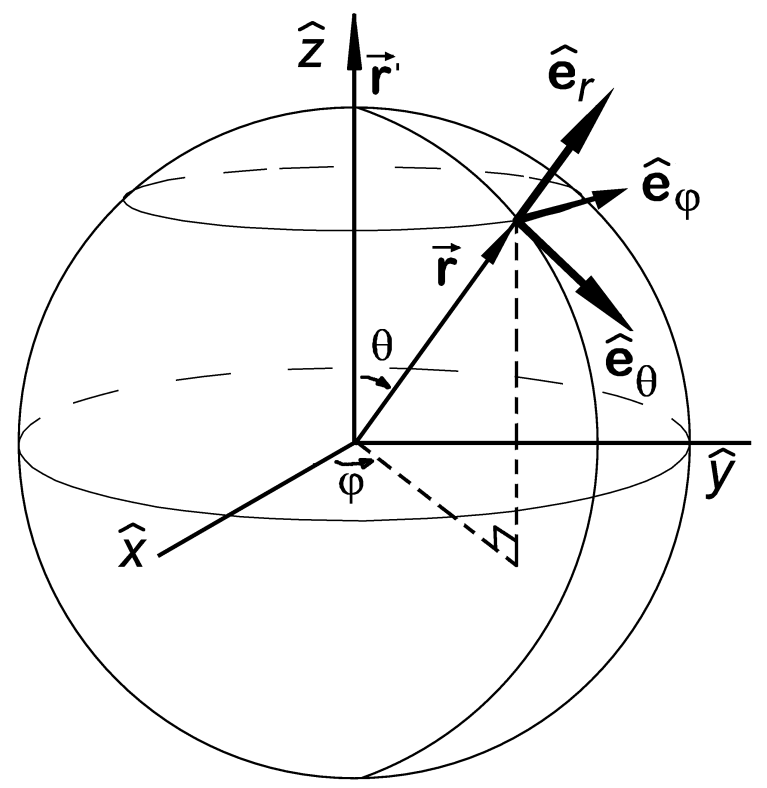

Figure 1.2: Two geocentric coordinate systems. Adapted from Wikipedia. 


$$
V=\frac{4 \pi G M^{\prime}}{r^{\prime}} \sum_{l=0}^{\infty}\left(\frac{1}{2 l+1}\right)\left(\frac{r}{r^{\prime}}\right)^{l} \sum_{m=-l}^{l} Y_{l}^{m *}\left(\theta^{\prime}, \phi^{\prime}\right) Y_{l}^{m}(\theta, \phi)
$$

Since table A.2 shows that $r^{0} Y_{0}^{0}$ is just $\sqrt{\frac{1}{4 \pi}}$, the $l=0$ term has a gradient of zero and therefore isn't associated with a gravitational force. Similarly, the $r^{1} Y_{1}^{0}$ term is a linear function of the coordinate $z=r \cos \theta$. The $r^{1} Y_{1}^{ \pm 1}$ terms are linear functions of the (complex) coordinates $x \pm i y=r \sin \theta \cos \phi \pm i r \sin \theta \sin \phi$ because $e^{ \pm i \phi}=\cos \phi \pm i \sin \phi$. The $l=1$ terms thus describe gravitational forces which don't vary over the surface of the Earth, so they move the Earth as a whole but don't cause tides.

Now consider the net force on a spherical Earth due to all the terms in $V$. The azimuthal symmetry of this problem can be exploited by using the addition theorem (equation A.27) to rewrite equation (1.5) as an infinite series of Legendre polynomials

$$
V=\frac{G M^{\prime}}{r^{\prime}} \sum_{l=0}^{\infty}\left(\frac{r}{r^{\prime}}\right)^{l} P_{l}(\cos \theta)
$$

where the angle between $\overrightarrow{\mathbf{r}^{\prime}}$ and $\overrightarrow{\mathbf{r}}$ is simply $\theta$ because $\overrightarrow{\mathbf{r}^{\prime}}$ points along the $\hat{\mathbf{z}}$ axis. The gradient of $V$ in spherical coordinates is (Jackson 1998)

$$
\begin{aligned}
\overrightarrow{\mathbf{f}}=\nabla V & =\frac{\partial V}{\partial r} \hat{\mathbf{e}_{\mathbf{r}}}+\frac{1}{r} \frac{\partial V}{\partial \theta} \hat{\mathbf{e}_{\theta}}+\frac{1}{r \sin \theta} \frac{\partial V}{\partial \phi} \hat{\mathbf{e}_{\phi}} \\
& =f_{r} \hat{\mathbf{e}_{\mathbf{r}}}+f_{\theta} \hat{\mathbf{e}_{\theta}}+f_{\phi} \hat{\mathbf{e}_{\phi}} .
\end{aligned}
$$

Equation (1.6) has no $\phi$ dependence, so $f_{\phi}=0$. The net force due to $V$ on a spherically symmetric Earth with radius $R$ and density $\rho(r)$ is

$$
\overrightarrow{\mathbf{F}}=\int_{0}^{R} \rho(r) r^{2} d r \int_{0}^{2 \pi} d \phi \int_{0}^{\pi} \sin \theta d \theta \overrightarrow{\mathbf{f}}
$$

Because the $\left(\hat{\mathbf{e}_{\mathbf{r}}}, \hat{\mathbf{e}_{\theta}}, \hat{\mathbf{e}_{\phi}}\right)$ basis vectors depend on $\overrightarrow{\mathbf{r}}, \overrightarrow{\mathbf{f}}$ should be rotated into the Cartesian 
basis $(\hat{\mathbf{x}}, \hat{\mathbf{y}}, \hat{\mathbf{z}})$ using equation (1.4), remembering that $f_{\phi}=0$ :

$$
\overrightarrow{\mathbf{f}}=\cos \phi\left(f_{r} \sin \theta+f_{\theta} \cos \theta\right) \hat{\mathbf{x}}+\sin \phi\left(f_{r} \sin \theta+f_{\theta} \sin \theta\right) \hat{\mathbf{y}}+\left(f_{r} \cos \theta-f_{\theta} \sin \theta\right) \hat{\mathbf{z}} .
$$

The closed integral in $\phi$ causes the $\hat{\mathbf{x}}$ and $\hat{\mathbf{y}}$ components of $\overrightarrow{\mathbf{F}}$ to vanish because of their $\cos \phi$ and $\sin \phi$ factors, respectively. The only remaining component $F_{z}$ can be rewritten using the definitions of $f_{r}$ and $f_{\theta}$ from equation (1.7):

$$
F_{z}=2 \pi \int_{0}^{R} \rho(r) r^{2} d r \int_{0}^{\pi} \sin \theta d \theta\left(\cos \theta \frac{\partial V}{\partial r}-\frac{\sin \theta}{r} \frac{\partial V}{\partial \theta}\right)
$$

Changing variables to $x=\cos \theta, d x=-\sin \theta d \theta$ yields

$$
F_{z}=2 \pi \int_{0}^{R} \rho(r) r^{2} d r \int_{-1}^{1} d x\left(x \frac{\partial V}{\partial r}+\frac{\left(1-x^{2}\right)}{r} \frac{\partial V}{\partial x}\right)
$$

Apply the derivatives to $V$ using equation (1.6) (dropping the $l=0$ terms in the process) and use recursion relation (A.13) to obtain

$$
F_{z}=\frac{2 \pi G M^{\prime}}{r^{\prime}} \sum_{l=1}^{\infty} \int_{0}^{R} \rho(r) d r \frac{(r)^{l+1}}{\left(r^{\prime}\right)^{l}} l \int_{-1}^{1} d x P_{l-1}(x)
$$

Because $P_{0}^{0}(x) \equiv P_{0}(x)=1$, the orthogonality of Legendre polynomials (see equation A.14) causes the integral over $x$ to collapse the sum over $l$ such that only $l=1$ is nonzero.

$$
F_{z}=\frac{4 \pi G M^{\prime}}{\left(r^{\prime}\right)^{2}} \int_{0}^{R} \rho(r) r^{2} d r
$$

Since the mass $\mathrm{M}$ of a spherically symmetric Earth is defined as

$$
M=4 \pi \int_{0}^{R} \rho(r) r^{2} d r
$$


the net force reduces to a familiar result

$$
\overrightarrow{\mathbf{F}}=F_{z} \hat{\mathbf{z}}=\frac{G M M^{\prime}}{\left(r^{\prime}\right)^{2}} \hat{\mathbf{z}}
$$

\subsection{The Gravitational Tidal Potential}

Because the $l=0,1$ terms of the gravitational potential don't cause tides, the gravitational tidal potential $V_{T}$ is defined without them

$$
V_{T}=\frac{4 \pi G M^{\prime}}{r^{\prime}} \sum_{l=2}^{\infty}\left(\frac{1}{2 l+1}\right)\left(\frac{r}{r^{\prime}}\right)^{l} \sum_{m=-l}^{l} Y_{l}^{m *}\left(\theta^{\prime}, \phi^{\prime}\right) Y_{l}^{m}(\theta, \phi)
$$

so its gradient yields tidal forces alone rather than a combination of tidal forces and forces

responsible for Earth's orbital motion. Since the tidal potential is usually evaluated on Earth's surface where $r=R \approx 6371 \mathrm{~km}$, incrementing $l$ by 1 reduces the magnitude of the tidal potential by a factor $\left(R / r^{\prime}\right)$. For the Moon, $\left(R / r_{M o o n}^{\prime}\right) \approx 1 / 60.3$; for the Sun, $\left(R / r_{\text {Sun }}^{\prime}\right) \approx 1 / 23481.1$. Therefore the infinite series can be truncated at $l=2$ and still be accurate to within $\sim 1.65 \%$ for the Moon and $\sim 0.00426 \%$ for the Sun.

$$
V_{T} \approx \frac{4 \pi G M^{\prime} r^{2}}{5 r^{\prime 3}} \sum_{m=-2}^{2} Y_{2}^{m *}\left(\theta^{\prime}, \phi^{\prime}\right) Y_{2}^{m}(\theta, \phi)
$$

Because $V_{T}$ is real, the sum over $m$ can be collapsed to $[0, l]$ as shown in equation (A.24)

$$
V_{T} \approx \frac{4 \pi G M^{\prime} r^{2}}{5 r^{\prime 3}} \mathbf{R e}\left[\sum_{m=0}^{2}\left(2-\delta_{m 0}\right) Y_{2}^{m *}\left(\theta^{\prime}, \phi^{\prime}\right) Y_{2}^{m}(\theta, \phi)\right]
$$

Consider the geocentric Earth-fixed coordinate system shown in figure 1.3, where both the Earth and the basis vectors rotate at frequency $\Omega$. The angle $\phi^{\prime}(t)$ (relative to $\phi^{\prime}$ at some epoch $t=0$ ) between the $\hat{\mathbf{x}}$ axis and the Moon's position is given in equation (1.19), where 


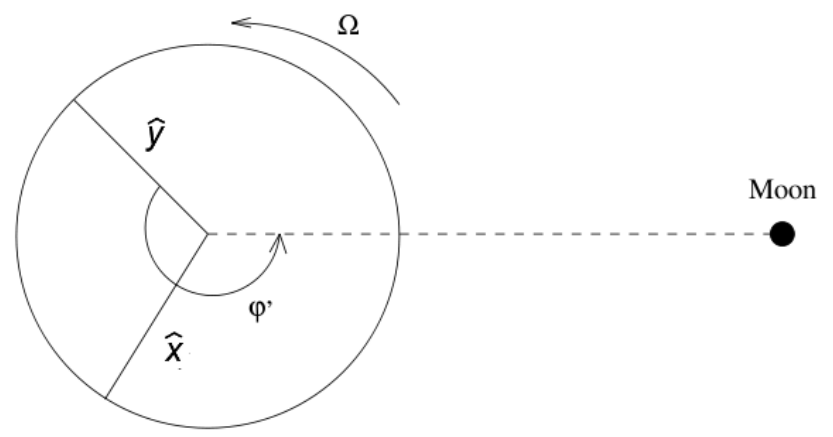

Figure 1.3: A geocentric Earth-fixed coordinate system. Adapted from Prof. John Wahr's course notes on Geodesy and Gravity at http://samizdat.mines.edu/geodesy/ .

$\alpha(t)$ describes the motion of the Moon in inertial space

$$
\phi^{\prime}(t)-\phi^{\prime}(t=0)=-\Omega t+\alpha(t) .
$$

This angle $\phi^{\prime}(t)$ can be substituted into equation (1.18) to obtain an expression for the tidal potential as measured at a single point on Earth's rotating surface:

$$
V_{T} \approx \frac{4 \pi G M^{\prime} r^{2}}{5 r^{\prime 3}} \mathbf{R e}\left[\sum_{m=0}^{2}\left(2-\delta_{m 0}\right) Y_{2}^{m *}\left(\theta^{\prime}, \alpha\right) e^{i m \Omega t} Y_{2}^{m}(\theta, \phi)\right] .
$$

The Moon orbits at angular rate $\alpha(t)$ which is $\sim 28$ times slower than the sidereal rotation rate of the Earth $\Omega$. Because $m$ multiplies the dominant frequency $\Omega$, different values of $m$ correspond to three tidal bands. For $m=2$, the time dependence is dominated by $e^{2 i \Omega t}$ which drives semi-diurnal tides with periods of about 12 hours. For $m=1$, the time dependence is dominated by $e^{i \Omega t}$ which drives diurnal tides with periods of about 24 hours.

The time dependence of $m=0$, on the other hand, isn't a result of the Earth's rotation. Instead, these long-period tides result from variations in r' (from the ellipticity of the Moon's orbit) and the associated changes in $\alpha(t)$ as the Moon's orbital velocity changes, as well as variations in $\theta^{\prime}$ due to the inclination of the Moon's orbit with respect to Earth's equatorial 
plane.

These time dependencies are conventionally approached by expanding the real function $V_{T}(R, \theta, \phi, t)$ (defined on the surface of the Earth with mean radius $r=R$ ) into Stokes coefficients as in equation (A.25):

$$
\begin{aligned}
\frac{V_{T}(R, \theta, \phi, t)}{g} & =\sum_{l=2}^{\infty} \sum_{m=0}^{l} P_{l}^{m}(\cos \theta)\left[C_{l}^{m}(t) \cos (m \phi)+S_{l}^{m}(t) \sin (m \phi)\right] \\
C_{l}^{m}(t) & =\frac{G M^{\prime}}{g r^{\prime}}\left(\frac{R}{r^{\prime}}\right)^{l}\left(2-\delta_{m 0}\right) \frac{(l-m) !}{(l+m) !} P_{l}^{m}\left(\cos \theta^{\prime}\right) \cos \left(m \phi^{\prime}\right) \\
S_{l}^{m}(t) & =\frac{G M^{\prime}}{g r^{\prime}}\left(\frac{R}{r^{\prime}}\right)^{l}\left(2-\delta_{m 0}\right) \frac{(l-m) !}{(l+m) !} P_{l}^{m}\left(\cos \theta^{\prime}\right) \sin \left(m \phi^{\prime}\right)
\end{aligned}
$$

The $C_{l}^{m}$ 's and $S_{l}^{m}$ 's are divided by the mean gravitational acceleration on the surface of the Earth $\left(g=9.80665 \mathrm{~m} / \mathrm{s}^{2}\right)$ so they have units of length. Notice that $C_{l}^{m}(t)$ and $S_{l}^{m}(t)$ only depend on the coordinates $\left(r^{\prime}, \theta^{\prime}, \phi^{\prime}\right)$ of the mass $\mathrm{M}^{\prime}$, and not the coordinates $(\theta, \phi)$ where the tidal potential is measured. The mean radius of Earth $R$ can be replaced by multiplying $C_{l}^{m}(t)$ and $S_{l}^{m}(t)$ by $(r / R)^{l}$, which yields the tidal potential at any radius $r$. Also, the time dependence of $V_{T}(R, \theta, \phi, t)$ is entirely contained within the $C_{l}^{m}(t)$ and $S_{l}^{m}(t)$ functions.

The relative magnitudes of tides due to different astronomical bodies can be estimated from the $M^{\prime} /\left(r^{\prime}\right)^{l+1}$ factors in the Stokes coefficients $C_{l}^{m}(t)$ and $S_{l}^{m}(t)$. For $l=2$, $\left(M^{\prime} / r^{\prime 3}\right)_{M o o n} \approx 2.1775\left(M^{\prime} / r^{\prime 3}\right)_{\text {Sun }} ;$ for $l=3,\left(M^{\prime} / r^{\prime 4}\right)_{M o o n} \approx 847.40\left(M^{\prime} / r^{\prime 4}\right)_{\text {Sun }}$. Because solar tides shrink faster than lunar tides as the degree $l$ increases, many developments of the tidal potential truncate solar tides at a lower degree than lunar tides. For instance, Cartwright and Tayler (1971); Cartwright and Edden (1973) includes lunar tides up to degree $l=3$ and solar tides to degree $l=2$. More recent work such as Hartmann and Wenzel (1995) includes lunar tides up to degree $l=6$, solar tides to degree $l=3$, and tides due to Mercury, Venus, Mars, Jupiter and Saturn to degree $l=2$. They also take into account the potential due to the Earth's flattening with the Moon and Sun, which alters the tidal potential by $\sim 5 \times 10^{-5}$ (Dahlen 1993). 


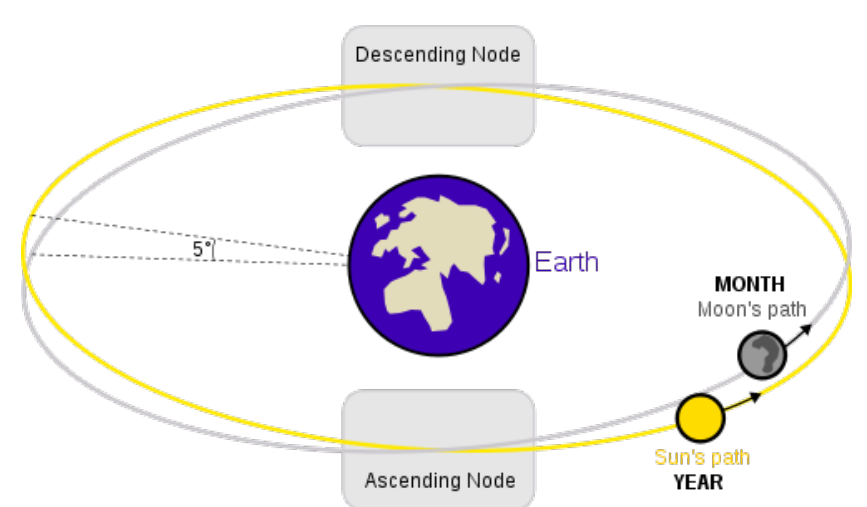

Figure 1.4: The lunar node. Adapted from Wikipedia.

\subsection{Harmonic Analysis of the Tidal Potential}

The tidal potential on the surface of the Earth $V_{T}(R, \theta, \phi, t)$ depends on time through the Stokes coefficients $C_{l}^{m}(t)$ and $S_{l}^{m}(t)$, which depend on the motion of the Moon and Sun (and other planets, to a much smaller extent) in a geocentric coordinate system. These positions are periodic not only because the Earth and Moon repeatedly traverse their orbits, but also because the orbits themselves precess on longer timescales. These periodicities allow the positions of the Moon and Sun to be accurately represented by harmonic functions of the relevant orbital frequencies, and linear combinations of those frequencies. Functions of the positions of the Moon and Sun such as $C_{l}^{m}(t)$ and $S_{l}^{m}(t)$ are therefore also accurately represented by harmonic functions of linear combinations of the relevant orbital frequencies (Darwin 1883; Doodson 1921).

The Earth-Moon-Sun system involves motion in three separate planes. The Earth's orbit defines the ecliptic plane, which is inclined with respect to Earth's equatorial plane by $23^{\circ} 27^{\prime}$. Similarly, the Moon's orbit is inclined with respect to the ecliptic plane by $5^{\circ} 08^{\prime}$. Any two non-parallel planes intersect along a line, and that line intersects the orbits defining those planes at an ascending (descending) node where the astronomical object crosses to the north (south) of the ecliptic. For example, figure 1.4 defines the lunar node.

Another reference point necessary to describe the motion of the Sun and Moon in a 
geocentric coordinate system is the perigee (closest approach) of each astronomical body. ${ }^{2}$ Figure 1.5 projects the following reference points $\left(\gamma, K, M, S, P_{M}, P_{S}\right)$ onto the ecliptic plane to define their directions relative to Earth without trying to specify their actual positions.

$\gamma$ - Vernal equinox (Ascending node of the ecliptic/equatorial planes)

$K$ - Lunar node (Ascending node of the lunar/ecliptic planes)

$M$ - The Moon's center of mass

$S$ - The Sun's center of mass

$P_{M}$ - Perigee of the Moon's orbit

$P_{S}$ - Perigee of the Sun's "orbit"

The angular positions of these reference points are usually measured relative to the vernal equinox because it's assumed to be fixed in inertial space. Figure 1.6 uses the vernal equinox and Earth's Greenwich meridian $(G)$ to define the following angles $\left(\phi, H, H^{\prime}, S T, \theta_{g}, \alpha, \alpha^{\prime}\right)$ in the equatorial plane.

$$
\begin{aligned}
& G P=\phi-\text { Geographic longitude of observer at point } P \\
& M G=H-\text { The Moon's Greenwich hour angle } \\
& S G=H^{\prime}-\text { The Sun's Greenwich hour angle } \\
& \gamma P=S T-\text { Local mean sidereal time } \\
& \gamma G=\theta_{g}-\text { Local mean Greenwich time } \\
& \gamma M=\alpha-\text { The Moon's right ascension } \\
& \gamma S=\alpha^{\prime}-\text { The Sun's right ascension }
\end{aligned}
$$

Since a mean lunar (solar) day is defined by the average time interval between consecutive returns of the Moon (Sun) to the local meridian, the Greenwich hour angle $H\left(H^{\prime}\right)$ can be used to define mean lunar (solar) time $\tau\left(\tau^{\prime}\right)$

\footnotetext{
${ }^{2}$ Of course, in a heliocentric coordinate system, the perigee of the Sun's "orbit" is known as the perihelion of the Earth's orbit.
} 


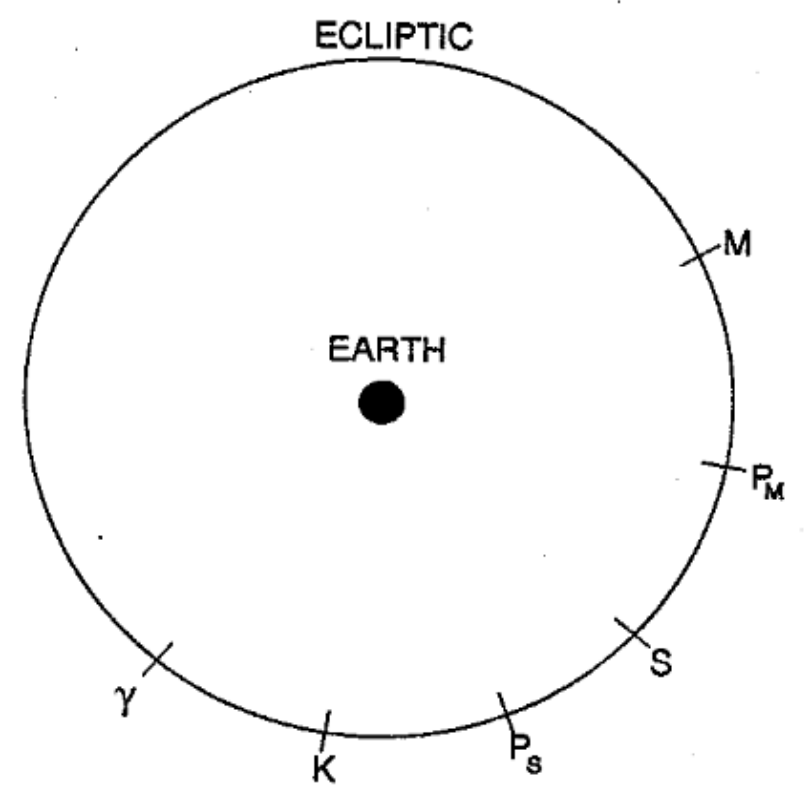

Figure 1.5: Reference points projected onto the ecliptic plane that define the orbital configuration of the EarthMoon-Sun system. Adapted from Desai (1996).

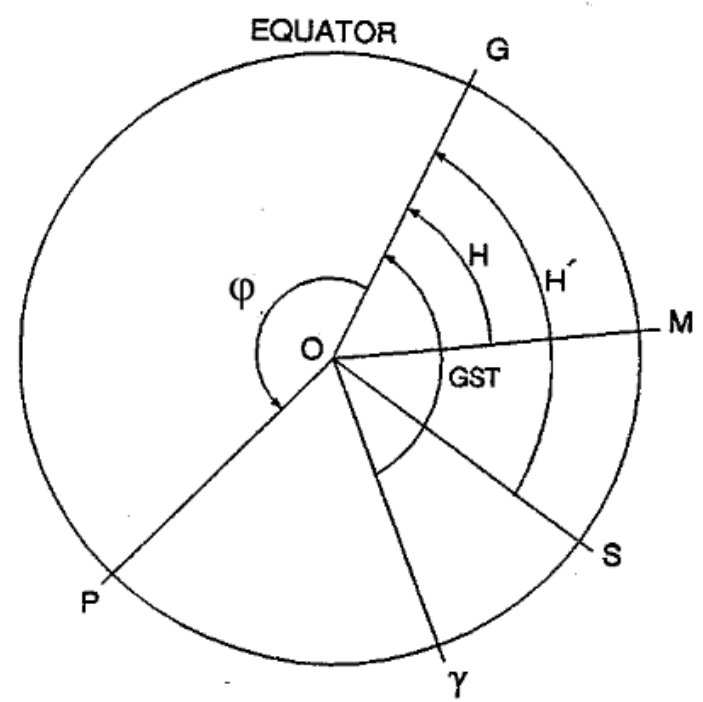

Figure 1.6: Angles between the vernal equinox $\gamma$, Greenwich meridian $G$, observer position $P$, Moon $M$ and Sun $S$ as measured in the equatorial plane using an Earthfixed geocentric coordinate system. Adapted from Desai (1996). 


$$
\begin{gathered}
\left.\tau=H+12 \text { hours }=H+180^{\circ} \text { (Mean lunar time }\right) \\
\left.\tau^{\prime}=H^{\prime}+12 \text { hours }=H^{\prime}+180^{\circ} \text { (Mean solar time }\right)
\end{gathered}
$$

The mean solar time $\tau^{\prime}$ is also known as universal time $t$, and the $180^{\circ}$ term is included so that $\tau^{\prime}=t=0$ at midnight over Greenwich. Either $\tau$ or $\tau^{\prime}$ could be used to transform angles measured in the ecliptic plane to the geocentric Earth-fixed coordinate system in the Earth's equatorial plane. Doodson (1921) chose to use the mean lunar time $\tau$, and defined the orbital configuration of the Earth-Moon-Sun system using the following six astronomical arguments.

$$
\begin{aligned}
\tau & =H+180^{\circ}-\text { Mean lunar time } \\
s & =\gamma M \quad-\text { Mean longitude of the Moon } \\
h & =\gamma S-\text { Mean longitude of the Sun } \\
p & =\gamma P_{M}-\text { Mean longitude of lunar perigee } \\
N^{\prime} & =-\gamma K \quad-\text { Negative mean longitude of ascending lunar node } \\
p^{\prime} & =\gamma P_{S}-\text { Mean longitude of solar "perigee" }
\end{aligned}
$$

These angles were defined in this manner by Doodson (1921) because their frequencies are always positive. Figure 1.6 shows that $H+\gamma M=H^{\prime}+\gamma S$, which means that lunar time can be defined as

$$
\tau=\tau^{\prime}+h-s
$$

The six Doodson astronomical arguments (collectively called $\Psi_{i}(t)$, where $\mathrm{i}=1,6$ ) can be calculated using quartic expressions of the elapsed universal time since the reference epoch $t=0$. Here the reference epoch is J2000, which corresponds to January 1, 2000 at 12:00PM GMT and Julian date 2451545.0. 


$$
\Psi_{i}(t)=\Psi_{i}(t=0)+\omega_{i} t+c_{i} t^{2}+d_{i} t^{3}+f_{i} t^{4}
$$

Table 1.1 lists the constants used in equation (1.24), which are taken from Simon et al. (1994). Note that only 5 of the 6 Doodson arguments are independent because of equation (1.23). The reference phase $\Psi_{1}(t=0)$, frequency $\omega_{1}$ and coefficients $c_{1}, d_{1}, f_{1}$ can be obtained by taking time derivatives of equation (1.23) and evaluating at $\tau^{\prime}=t=0$ :

$$
\begin{aligned}
\Psi_{1}(t=0) & =180^{\circ}+\Psi_{3}(t=0)-\Psi_{2}(t=0) \\
\omega_{1} & =\omega_{0}+\omega_{3}-\omega_{2} \\
c_{1} & =c_{3}-c_{2} \\
d_{1} & =d_{3}-d_{2} \\
f_{1} & =f_{3}-f_{2} .
\end{aligned}
$$

The extra $180^{\circ}$ term in the definition of $\Psi_{1}(t=0)$ compensates for the fact that mean solar time $\tau$ is defined in equation (1.22) to be zero at midnight at Greenwich, whereas the J2000 reference epoch is zero at noon. The frequency $\omega_{0}$ is the time derivative of $\tau^{\prime}$, which is defined to be a constant $360^{\circ}$ per solar day, or $131,490,000^{\circ}$ per Julian millenia to match the units in table 1.1.

The Stokes coefficients $C_{l}^{m}(t)$ and $S_{l}^{m}(t)$ defining the tidal potential in equation (1.21) can therefore be expressed as a harmonic series

$$
\begin{aligned}
& C_{l}^{m}(t)=\sum_{j} H_{l m j} \cos \left(\omega_{l m j} t+\beta_{l m j}\right) \\
& S_{l}^{m}(t)=-\sum_{j} H_{l m j} \sin \left(\omega_{l m j} t+\beta_{l m j}\right) .
\end{aligned}
$$

There are infinitely many frequencies $\omega_{l m j}$ because they're linear combinations of the six 


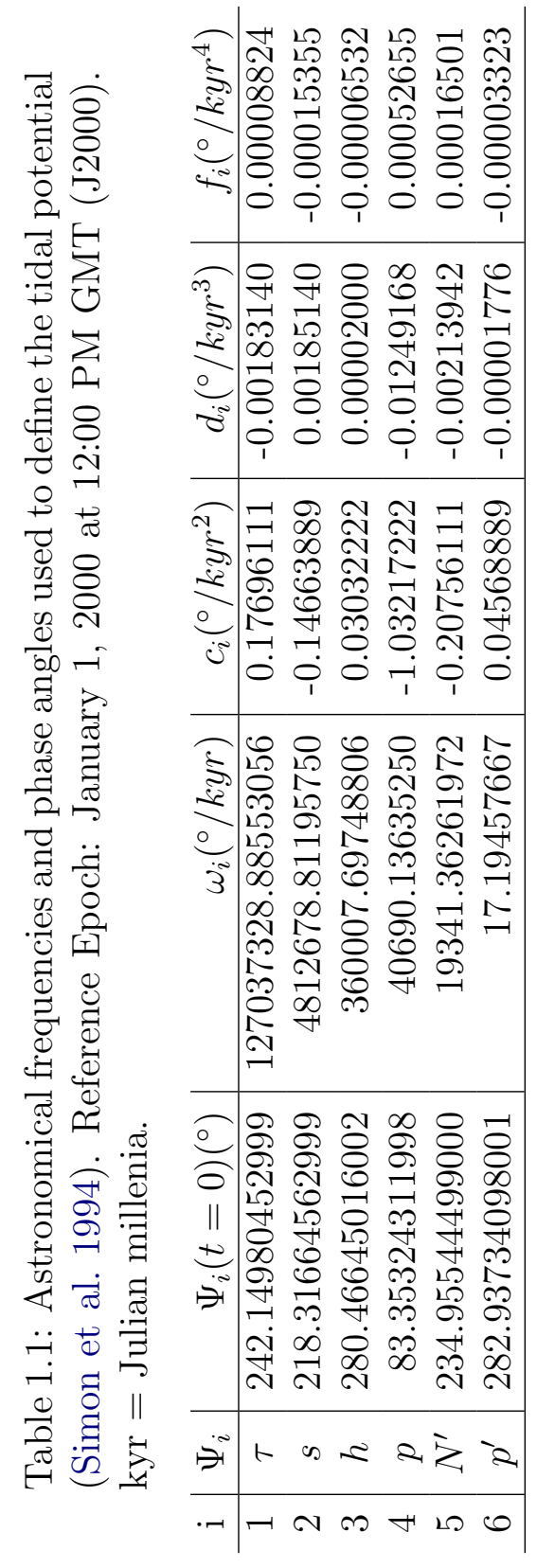


fundamental frequencies in table 1.1, where each frequency is multiplied by an integer. ${ }^{3}$ These integers are collectively known as the Doodson number defining the frequency $\omega_{l m j}$, which is conventionally displayed as

$$
d_{1} d_{2} d_{3} \cdot d_{4} d_{5} d_{6}
$$

where $d_{1}$ is called the tidal species and is equal to the order $m$. Thus long period tides have $d_{1}=0$, diurnal tides have $d_{1}=1$, and semi-diurnal tides have $d_{1}=2$. The two digit number $d_{1} d_{2}$ is known as the tidal group number; $d_{1} d_{2} d_{3}$ is referred to as the tidal constituent number. Table 1.2 lists the Doodson numbers and associated Darwinian symbols for degree 2 tidal constituents. The $H_{l m j}$ 's are calculated using the Cartwright and Tayler (1971) development, and help to compare the relative magnitudes of different tidal constituents.

The tidal potential in equation (1.21) can be rewritten using (1.26) as

$$
\frac{V_{T}(R, \theta, \phi, t)}{g}=\sum_{l=2}^{\infty} \sum_{m=0}^{l} H_{l m j} \sqrt{\frac{(2 l+1)(l-m) !}{4 \pi(l+m) !}} P_{l}^{m}(\cos \theta) \cos \left(m \phi+\omega_{l m j} t+\beta_{l m j}\right)
$$

where the negative sign in equation (1.26)'s definition of $S_{l}^{m}(t)$ allows the cosine addition formula to combine the sin and cos terms in equation (1.21). The argument $\omega_{l m j} t+\beta_{l m j}$ is calculated using the Doodson numbers and the angles defining the Earth-Moon-Sun system:

$$
\begin{aligned}
& \omega_{l m j} t+\beta_{l m j}= d_{1} \tau+\left(d_{2}-5\right) s+\left(d_{3}-5\right) h+\left(d_{4}-5\right) p \\
&+\left(d_{5}-5\right) N^{\prime}+\left(d_{6}-5\right) p^{\prime}+\delta(l, m) \frac{\pi}{2} . \\
& \delta(l, m)=\left\{\begin{array}{l}
1 \text { if } 1+\mathrm{m} \text { is odd } \\
0 \text { if } \mathrm{l}+\mathrm{m} \text { is even }
\end{array}\right.
\end{aligned}
$$

\footnotetext{
${ }^{3}$ An infinity of tidal frequencies may be intimidating, but keep in mind that the coefficients are integers. Therefore to represent the full tidal spectrum you merely need a computer with a countably infinite amount of memory.
} 
Table 1.2: Tidal frequencies and amplitudes for tides in the Cartwright-Tayler-Edden development. Adapted from Desai (1996).

\begin{tabular}{|c|c|c|c|}
\hline $\begin{array}{c}\text { Darwinian } \\
\text { Symbol }\end{array}$ & $\begin{array}{l}\text { Doodson } \\
\text { Number }\end{array}$ & $\begin{array}{c}\text { Frequency } \\
\omega_{1 m j} \text { (cycles/day) }\end{array}$ & $\begin{array}{l}\text { Amplitude } \\
H_{1 m j}(\mathrm{~cm})\end{array}$ \\
\hline $\mathrm{S}_{\mathrm{a}}$ & 056.554 & 0.00273778 & -0.492 \\
\hline $\mathrm{S}_{\mathrm{sa}}$ & 057.555 & 0.00547582 & -3.098 \\
\hline $\mathrm{M}_{\mathrm{m}}$ & 065.455 & 0.03629165 & -3.518 \\
\hline $\mathrm{M}_{\mathrm{sf}}$ & 073.555 & 0.06772638 & -0.583 \\
\hline $\mathrm{M}_{\mathrm{f}}$ & 075.555 & 0.07320220 & -6.661 \\
\hline $\mathrm{M}_{\mathrm{t}}$ & 085.455 & 0.10949385 & -1.276 \\
\hline $2 Q_{1}$ & 125.755 & 0.85695241 & -0.664 \\
\hline$\sigma_{1}$ & 127.555 & 0.86180932 & -0.802 \\
\hline $\mathrm{Q}_{1}$ & 135.655 & 0.89324406 & -5.019 \\
\hline$\rho_{1}$ & 137.455 & 0.89810097 & -0.954 \\
\hline $\mathrm{O}_{1}$ & 145.555 & 0.92953571 & -26.216 \\
\hline$\tau_{1}$ & 147.555 & 0.93501153 & 0.343 \\
\hline $\mathrm{M}_{1}$ & 155.655 & 0.96644626 & 2.062 \\
\hline$\chi_{1}$ & 157.455 & 0.97130317 & 0.394 \\
\hline$\pi_{1}$ & 162.556 & 0.99452431 & -0.713 \\
\hline $\mathrm{P}_{1}$ & 163.555 & 0.99726209 & -12.201 \\
\hline $\mathrm{S}_{1}$ & 164.556 & 1.00000013 & 0.289 \\
\hline $\mathrm{K}_{1}$ & 165.555 & 1.00273791 & 36.867 \\
\hline$\psi_{1}$ & 166.554 & 1.00547569 & 0.293 \\
\hline$\phi_{1}$ & 167.555 & 1.00821373 & 0.525 \\
\hline$\theta_{1}$ & 173.655 & 1.03417265 . & 0.395 \\
\hline$J_{1}$ & 175.455 & $1.03902956 \mathrm{i}$ & 2.062 \\
\hline $\mathrm{OO}_{1}$ & 185.555 & 1.07594011 & 1.129 \\
\hline$\epsilon_{2}$ & 227.655 & 1.82825558 & 0.467 \\
\hline $2 \mathrm{~N}_{2}$ & 235.755 & 1.85969032 & 1.601 \\
\hline$\mu_{2}$ & 237.555 & 1.86454723 & 1.932 \\
\hline $\mathrm{N}_{2}$ & 245.655 & 1.89598197 & 12.099 \\
\hline$\nu_{2}$ & 247.455 & 1.90083888 & 2.298 \\
\hline $\mathrm{M}_{2}$ & 255.555 & 1.93227362 & 63.194 \\
\hline$\lambda_{2}$ & 263.655 & 1.96370835 & -0.466 \\
\hline $\mathrm{L}_{2}$ & 265.455 & 1.96856526 & -1.786 \\
\hline $\mathrm{T}_{2}$ & 272.5506 & 1.99726222 & 1.718 \\
\hline $\mathrm{S}_{2}$ & 273.555 & 2.00000000 & 29.402 \\
\hline $\mathrm{R}_{2}$ & 274.554 & 2.00273778 & -0.246 \\
\hline $\mathrm{K}_{2}$ & 275.555 & 2.00547582 & 7.993 \\
\hline$\eta_{2}$ & 285.455 & 2.04176747 & 0.447 \\
\hline
\end{tabular}


Notice that each $d_{i}$ except $d_{1}$ has 5 subtracted from it. Doodson (1921) chose this convention to avoid writing negative signs in the Doodson number. The term $\delta(l, m) \frac{\pi}{2}$ is added to conform to the convention established by Cartwright and Tayler (1971).

Calculating the tidal potential $V_{T}(R, \theta, \phi, t)$ doesn't translate directly into knowledge of the actual amplitudes of the ocean tides because the admittance of the oceans (the complex proportionality factor between the tidal potential and the actual amplitude) varies significantly due to complications such as irregular bathymetry and coastlines. As a result, tide models list empirically-derived values of ocean tide amplitudes $A(\theta, \phi)$ for each tidal constituent. Because ocean tides aren't necessarily in phase with the tidal potential, tide models also list Greenwich phase lags $G(\theta, \phi)$ for each tidal constituent. The height of a particular tidal constituent at coordinates $(\theta, \phi)$ and time $t$ is calculated using

$$
\operatorname{Height}_{l m j}(\theta, \phi, t)=A(\theta, \phi) \cos \left[\omega_{l m j} t+\beta_{l m j}+\delta_{l m j} \pi-G(\theta, \phi)\right]
$$

where the term $\delta_{l m j}$ insures that a Greenwich phase lag of zero (the phase lag of body tides) corresponds to a maximum in the tidal potential over Greenwich instead of a minimum. For $l=2$, this term is given by:

$$
\delta_{2 m j}=\left\{\begin{array}{l}
1 \text { when }(-1)^{m+\delta_{m 0}} H_{2 m j}<0 \\
0 \text { when }(-1)^{m+\delta_{m 0}} H_{2 m j}>0 .
\end{array}\right.
$$

Table 1.3 lists the periods corresponding to the six Doodson angles, and provides explicit formulas for the frequencies of major tidal constituents to illustrate the use of Doodson numbers. Notice that the $K_{1}$ and $K_{2}$ tides are special cases where the Sun and Moon both produce tides at the same frequencies. The $K_{1}$ tide is the sum of a lunar tide $K_{1}^{M}$ and a solar tide $K_{1}^{S}$ which both have angular arguments (see equation 1.28) equal to that of Greenwich mean sidereal time. Thus the $K_{1}$ tide's forcing potential is stationary in inertial 
space, resulting in a tidal period equal to one sidereal day.

The Cartwright and Tayler (1971) development includes degree 3 lunar tides and degree 2 solar tides, and the resulting tidal spectra are shown in figure 1.7. It includes 104 longperiod tides, 162 diurnal tides and 119 semidiurnal tides. More recent work such as Hartmann and Wenzel (1995) includes degree 6 lunar tides, degree 3 solar tides and degree 2 tides for other planets, and results in a total of 12,935 tides. Each additional degree $l$ adds a new tidal band around frequency $l \Omega$, and contributes new tidal constituents to previously defined tidal bands around frequencies $m \Omega$ (for $0 \leq m<l$ ) which increases their spectral densities. Kudryavtsev (2004) expands the tidal potential as a Poisson series, and includes $\sim 27,000$ terms. Other approaches such as the response method (Munk and Cartwright 1966) don't require explicit knowledge of the tidal frequencies, instead relying on the assumption that the ocean's complex admittance is a slowly varying function of frequency. 


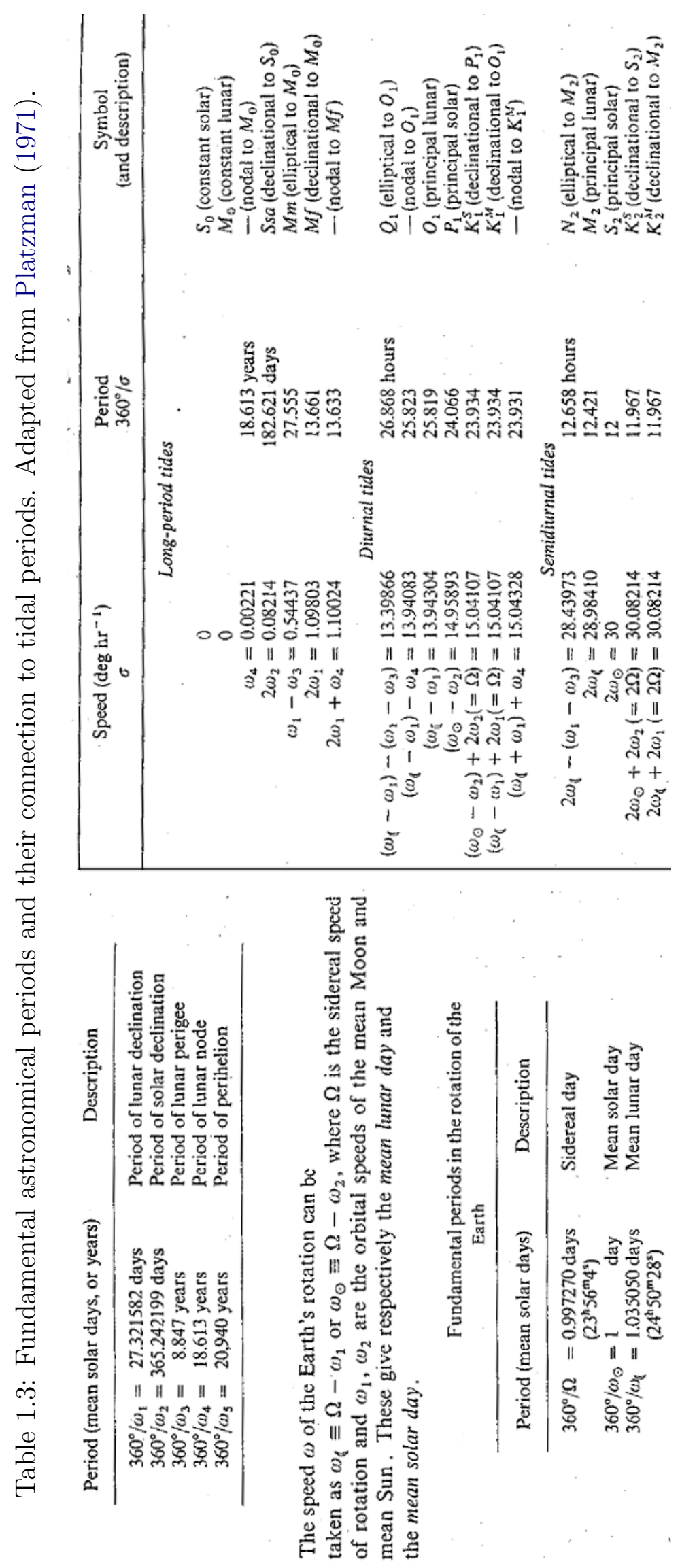



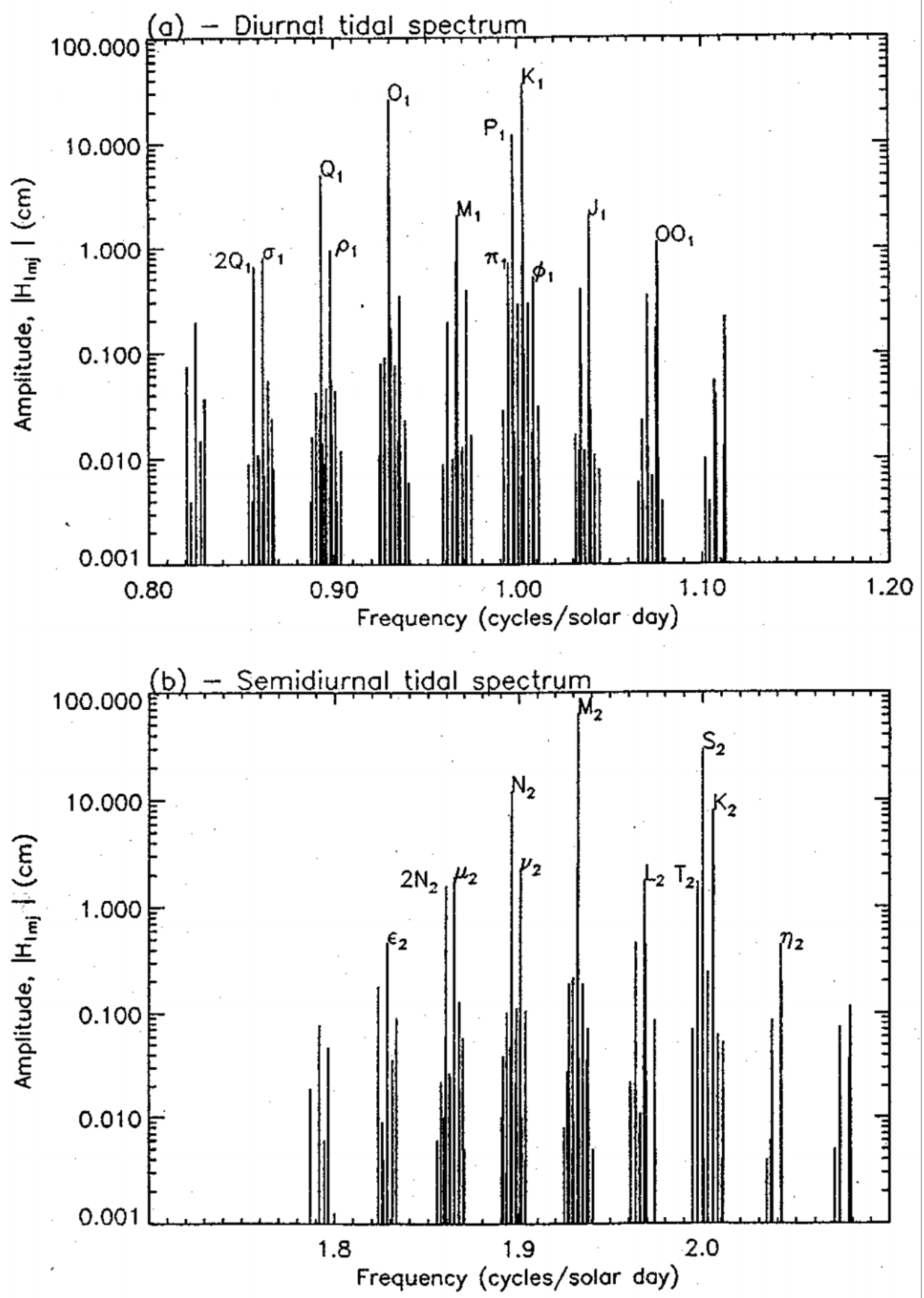

Figure 1.7: Diurnal and semidiurnal tidal spectra in the Cartright-Tayler-Edden development. Adapted from Desai (1996). 


\section{Chapter 2}

\section{GRACE}

Earth's gravity field varies with time due to contributions from such things as postglacial rebound, continental water storage, tides, atmospheric circulation, glacier thinning and non-tidal ocean circulation (Wahr 2007). Tide models, truncated to some maximum spherical harmonic degree, are routinely used to remove tidal signals from the raw GRACE data during processing. Accurate models of body tides are available (McCarthy and Petit 2004), but ocean tides are more complex due to the irregular drag and resonances caused by bathymetry and coastlines interacting with effects of the Earth's rotation. Residual tidal errors alias into GRACE monthly fields (Han et al. 2005; Moore and King 2008; Ray et al. 2009; Egbert et al. 2009; Melachroinos et al. 2009).

GRACE measures gravity fluctuations using two satellites in the same low $(\sim 460 \mathrm{~km})$ polar orbit. (Tapley et al. 2004a) One satellite is $\sim 220 \mathrm{~km}$ ahead of the other, and they're linked by a microwave ranging system that continuously measures their relative separation distance. A gravity feature affects the leading satellite before the trailing satellite, which changes their separation distance. These data can thus be inverted to obtain the timevariable surface mass distribution. Many GRACE applications describe these mass variations using spherical harmonics, but this research uses mass concentrations ("mascons") (Luthcke et al. 2008) to parameterize variations in surface mass.

We give each mascon several degrees of freedom: a constant, a trend, and ampli-

tudes/phases at frequencies which can represent tides or hydrologic / cryospheric / oceanic 


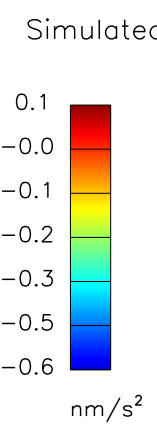

Figure 2.1: Simulated GRACE accelerations due to a mascon (marked by a red "X") at $61^{\circ} \mathrm{N}, 36^{\circ} \mathrm{E}$.

(non-tidal) processes. At first, the gravitational force from each mascon is approximated by assuming that all its mass is concentrated at a point at the center of the mascon; this approximation is later relaxed in section 3.2. Our approach solves for these mascon parameters using relative accelerations between the GRACE satellites. Two time derivatives of the range measurements are taken to provide relative acceleration. Figure 2.1 displays simulated accelerations due to a mascon at $61^{\circ} \mathrm{N}, 36^{\circ} \mathrm{E}$ which is modelled as a $10 \mathrm{~cm}$ thick layer of water spread over a rectangular area $230 \mathrm{~km}$ on each side. The blue feature demonstrates that the GRACE satellites accelerate towards each other when the mascon is between them, while the red features indicate that the satellites accelerate away from each other $\sim 6$ times slower when the mascon is north or south of the GRACE pair. The large east-west extent of this "footprint" allows GRACE to recover most diurnal and semi-diurnal tides because successive measurements occur more than twice in each tidal period (at most locations on Earth's surface) which satisfies the Nyquist-Shannon sampling theorem (Jerri 1977).

Differentiation of the range measurements introduces high frequency noise which is smoothed by a CRN filter (Wu et al. 2006) at the cost of attenuating signals due to shortscale features. The advantage of using acceleration data is that the acceleration at any instant is determined solely by forces acting on the two satellites at that instant. In contrast, separation distances or velocities are determined by forces acting in the past. An inversion 
Table 2.1: Tidal alias periods in GRACE data. Adapted from Ray and Luthcke (2006)

\begin{tabular}{ccrr}
\hline Tide & $\begin{array}{c}f \\
(\% / \text { day })\end{array}$ & $\begin{array}{r}f_{\text {alias }} \\
(\% \text { day })\end{array}$ & $\begin{array}{r}\text { Alias period } \\
\text { (days) }\end{array}$ \\
\hline$Q_{1}$ & 321.57 & 39.55 & 9.1 \\
$O_{1}$ & 334.63 & 26.48 & 13.6 \\
$P_{1}$ & 359.01 & 2.10 & 171.2 \\
$S_{1}$ & 360.00 & 1.12 & 322.1 \\
$K_{1}$ & 360.99 & 0.13 & 2725.4 \\
$J_{1}$ & 374.05 & 12.93 & 27.8 \\
$N_{2}$ & 682.55 & 39.68 & 9.1 \\
$M_{2}$ & 695.62 & 26.62 & 13.5 \\
$S_{2}$ & 720.00 & 2.24 & 161.0 \\
$K_{2}$ & 721.97 & 0.26 & 1362.7 \\
\hline
\end{tabular}

algorithm using accelerations doesn't need to integrate the mascons' gravitational forces over time, which reduces computation time and hardware requirements.

Using GRACE data to solve for tides requires careful consideration of the alias periods of major tides, which are listed in table 2.1. Note that the $K_{1}$ alias period is especially long at $\sim 7.5$ years. 


\section{Chapter 3}

\section{Methodology}

Approximately seven years (8 April 2002 to 30 July 2009) of GRACE inter-satellite accelerations (Case et al. 2002) recorded every 5 seconds when the satellites' midpoint is north of $50^{\circ} \mathrm{N}$ are matched with linearly interpolated positions of each satellite at those times. These accelerations have dealiasing products such as AOD1B (Flechtner 2003) subtracted, and are smoothed by a CRN filter with a 20 second cutoff. The complete FES2004 tide model (but without the $S_{1}$ fix (Scharroo 2006) or $K_{2}$ from FES2002 (Lyard et al. 2006)) is subtracted (Desai and Yuan 2006) after truncation at maximum spherical harmonic degree and order 90 .

The observed inter-satellite acceleration is proportional to the difference between the accelerations of each satellite projected onto the unit vector $(\hat{\mathbf{n}})$ pointing from GRACE 1 to GRACE 2. Defining $\overrightarrow{\mathbf{f}_{\mathbf{1}}}\left(t_{i}\right)$ as the force per unit mass exerted on GRACE 1 by the j'th mascon at time $t_{i}$, Newtonian point mass gravity defines partial derivatives of the i'th acceleration (measured at GRACE positions $\overrightarrow{\mathbf{r}_{\mathbf{1}}}\left(t_{i}\right), \overrightarrow{\mathbf{r}_{\mathbf{2}}}\left(t_{i}\right)$ ) with respect to the mass of the j'th mascon (at $\overrightarrow{\mathbf{r}_{\mathbf{j}}}$ ) by

$$
\begin{gathered}
\overrightarrow{\mathbf{f}_{\mathbf{1} \mathbf{j}}}\left(t_{i}\right)=\frac{G m_{j}\left(\overrightarrow{\mathbf{r}_{\mathbf{j}}}-\overrightarrow{\mathbf{r}_{\mathbf{1}}}\left(t_{i}\right)\right)}{\left|\overrightarrow{\mathbf{r}_{\mathbf{j}}}-\overrightarrow{\mathbf{r}_{\mathbf{1}}}\left(t_{i}\right)\right|^{3}} \\
\frac{\partial a\left(t_{i}\right)}{\partial m_{j}}=\left(\overrightarrow{\mathbf{f}_{\mathbf{2} \mathbf{j}}}\left(t_{i}\right)-\overrightarrow{\mathbf{f}_{\mathbf{1} \mathbf{j}}}\left(t_{i}\right)\right) \cdot \hat{\mathbf{n}} .
\end{gathered}
$$


Mass is converted to "equivalent thickness of water" over the mascon using a nominal density of seawater and the area of the mascon. When multiplied by time dependent factors at the times each acceleration is measured, equation (3.2) can represent the acceleration from a mascon's constant, trend, or sinusoidal behavior. The observed accelerations are related (Tapley et al. 2004b) to the mascon parameters by

$$
\overrightarrow{\mathbf{y}}=\mathbf{H} \overrightarrow{\mathbf{x}}+\vec{\epsilon}
$$

where $\overrightarrow{\mathbf{y}}$ is the observation vector of accelerations with size N corrupted with errors $\vec{\epsilon}, \overrightarrow{\mathbf{x}}$ is the state vector of mascon parameters with size $\mathrm{T}=\mathrm{M}^{*} \mathrm{P}(\mathrm{M}$ mascons, each with $\mathrm{P}$ temporal parameters), and $\mathbf{H}$ is the observation-state mapping matrix, filled with values calculated from equation (3.2). A least squares solution of $\overrightarrow{\mathbf{x}}$ in equation (3.3) is obtained by minimizing a performance index given by the $L_{2}$ norm of the errors $\vec{\epsilon}=\overrightarrow{\mathbf{y}}-\mathbf{H} \overrightarrow{\mathbf{x}}$. This is equivalent to solving

$$
\vec{b}=A \vec{x}
$$

where $\overrightarrow{\mathbf{b}}=\mathbf{H}^{T} \overrightarrow{\mathbf{y}}$ and $\mathbf{A}=\mathbf{H}^{T} \mathbf{H}$ is the symmetric but indefinite covariance matrix. Because $\mathbf{H}$ has $\mathrm{T}^{*} \mathrm{~N}$ entries (typically, $\mathrm{T}$ is $\sim 10^{4}$ and $\mathrm{N}$ is $\sim 10^{7}$ ), it's too large to fit into memory. Since $\mathbf{A}$ only has $T^{2}$ entries, it's $\sim 10^{3}$ times smaller than $\mathbf{H}$. This makes $\mathbf{A}$ more practical to compute but requires rearranging the sums implied in equation (3.4) to avoid the need to store $\mathbf{H}$ as an intermediate result.

The covariance matrix $\mathbf{A}$ is nearly singular, so this ill-posed problem is prone to numerical errors and thus requires regularization. Truncated singular value decomposition (Press et al. 1992) and Gaussian smoothing (Barmin et al. 2001) were tried, but Tikhonov damping (Aster et al. 2005) is faster, requires only a single adjustable parameter, and performs well in simulations (such as those described in chapter 4). Tikhonov damping is applied by adding 
a weighted identity matrix to the covariance matrix before using LU decomposition (Turing 1948) to solve

$$
\overrightarrow{\mathbf{b}}=(\mathbf{A}+\lambda \mathbf{I}) \overrightarrow{\mathbf{x}}
$$

This addition to the performance index penalizes solutions with large amplitudes, and so tends to smooth the output mascon field. A weighting factor of $\lambda=20$ was originally subjectively chosen because it produces output which isn't too noisy, and doesn't excessively attenuate continent-scale simulated signals, as discussed in chapter 4 . Later, the accelerations implied by the mascon field are subtracted from verification accelerations (see section 5.2) not used in the inversion. The relative variance reduction of the verification accelerations reaches a maximum when $\lambda$ is set between 19 and 21, thus supporting our original choice of $\lambda=20$.

One mascon is placed at the north pole and others form concentric circles around it with nearly equal areas, though small latitude-dependent deviations exist due to the need to have an integer number of grid points at each latitude. These areas should be similar because each mascon's mass (in kg) is converted to the equivalent thickness of water. Placing this conversion in $\mathbf{A}$ avoids numerical problems inherent to large differences in the magnitudes of the observation and state vectors.

The conversion from mass to water thickness depends on the assumed density of water, so errors in that density affect the accuracy of the inverted mascon field relative to tide gauges. Here that density is assumed to be a uniform $1035 \mathrm{~kg} / \mathrm{m}^{3}$ because tides move the entire water column which has a more stable temperature and salinity than surface water, and because the effects of a laterally (Steele and Ermold 2007) and temporally (Wang et al. 2010) varying density aren't likely to rise above the current GRACE noise floor. However, future studies may need to account for local variations and small annual density fluctuations (Willis et al. 2008) that would otherwise modulate the estimate of water thickness. 
This research doesn't correct for the fact that GRACE can't detect changes in the Earth's center of mass relative to the geometric center of the Earth. This problem is alternatively described as an inability to reconstruct the degree 1 spherical harmonic terms of the Earth's surface mass distribution using GRACE data alone (Swenson et al. 2008).

\subsection{Zone of Influence}

Summing the contributions of all accelerations for every entry in the covariance matrix would take months on a typical desktop computer. To increase speed, each acceleration is only allowed to contribute to a mascon solution, for mascons lying within a certain distance (measured from the mascon center to the projection of the satellites' midpoint onto a spherical Earth). This "zone of influence" moves with the satellites and is different for each acceleration measurement, so in principle it's necessary to calculate the distances from the satellites' midpoint to all mascons at all times to determine which mascons lie within the zone of influence. Because there are millions of acceleration measurements, this strategy wouldn't provide a significant speedup.

However, each acceleration value can be matched to the closest mascon (of which there are usually merely thousands rather than millions) and the zone of influence is then determined from values that have been pre-calculated using that mascon's position, rather than determined directly from the position of the satellites' midpoint. This approximation reduces the number of required calculations by a factor of $\sim 10^{3}$ and introduces negligible errors because any omitted mascons are already far away from the acceleration measurement in question.

This algorithm's only parameter is the zone radius; smaller values dramatically reduce the computation time at the cost of increasing error by ignoring mascons that contribute substantially to the measured accelerations. The simulations in chapter 4 and real inversions in chapter 5 use a zone radius of $2050 \mathrm{~km}$, which introduces relative errors in the output mascon field that are less than $10 \%$ of the maximum mascon value. The simulated acceler- 
ations in figure 2.1 suggest that choosing a zone radius too small to cover the significantly non-zero portion of that map will increase error, which is observed in figure 3.1.

\subsection{Support Grid Points}

One drawback of using Newtonian point mass gravity is that real tides are distributed over the surface of the Earth rather than being concentrated as geometric points. This inaccuracy is sometimes avoided by modeling the mascons as uniform disks, and computing their gravitational signatures as spherical harmonic expansions. These infinite expansions converge on a gravitational solution for a distributed mass. But truncating at a finite number of terms for numerical computation introduces non-local errors (i.e. creates an effective nonzero mass distribution outside the mascon.)

These errors can be circumvented by placing the point masses close enough together that GRACE isn't sensitive enough to distinguish them. Unfortunately, this approach requires more memory than our computers have available because the size of the covariance matrix scales as the square of the number of grid points, which scales as the inverse square of the grid spacing.

Our solution is to use support grid points to account for the distributed nature of the genuine water mass while avoiding non-local errors. Each main grid point has a set of support points associated with it. They evenly fill the space between main grid points with arbitrary spatial density. For example, in figure 3.2 the main grid points are shown in black and the colors of the support points indicate which main grid point they're associated with.

The support points' gravitational effects are summed together (weighted according to surface area so each support point has the same sea surface height as the main grid point) with the main grid point to produce a single entry in the covariance matrix. The mass which was previously assumed to be concentrated at the main grid point is now distributed over

the support points so that the equivalent thickness of water is spread more uniformly over the area of the mascon. Adding support points doesn't increase memory requirements, but 
(a) Input Trend

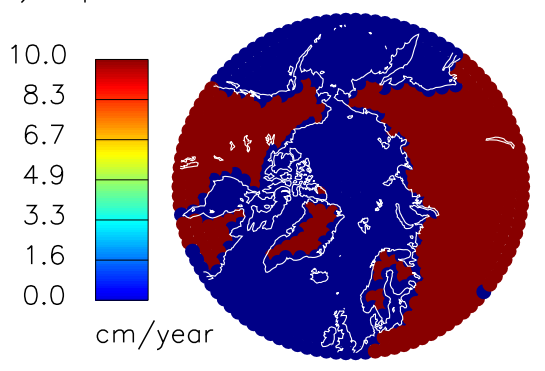

(c) Output Trend - $1450 \mathrm{~km}$ Zone

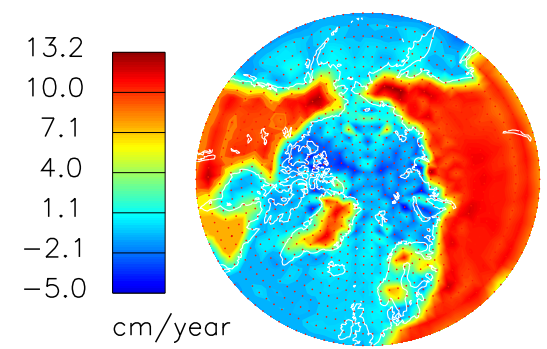

(b) Output Trend - 2050km Zone

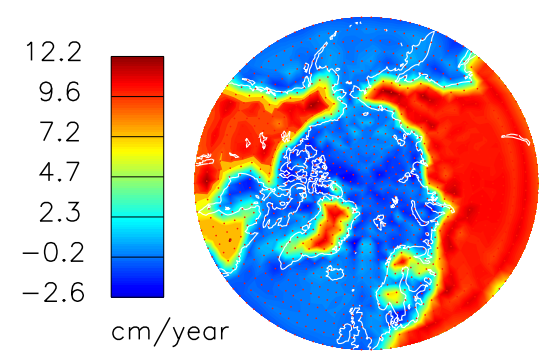

(d) Output Trend $-850 \mathrm{~km}$ Zone

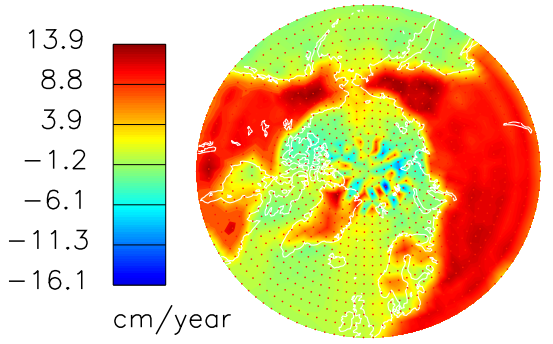

Figure 3.1: Simulation that tests the zone of influence algorithm; (a) is the input secular trend which is upward-continued to create a synthetic GRACE acceleration time series; (b) is the result of inverting those synthetic accelerations using a $2050 \mathrm{~km}$ zone of influence, a process which takes 5.5 hours on our computer; (c) uses $1450 \mathrm{~km}$ and takes 3 hours; (d) uses $850 \mathrm{~km}$ and takes 1 hour.

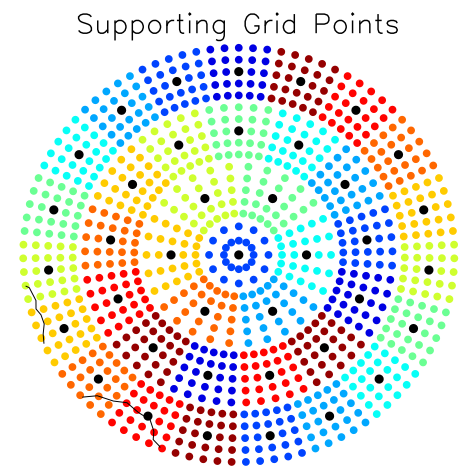

Figure 3.2: Main grid points are shown in black, separated by about $230 \mathrm{~km}$. Each main grid point is associated with 24 color-coded support points. 
also doesn't increase the spatial resolution of the resulting map.

Surprisingly, this code's runtime increases more slowly than linearly with respect to the number of support points. That's because the algorithm spends most of its time calculating entries in the covariance matrix $\mathbf{A}$, which is done after the support points are summed to produce entries in $\mathbf{H}$ and therefore doesn't depend on them. The centers of the mascons are separated by about $230 \mathrm{~km}$, and each mascon used in chapter 5 has 24 support points. Expanding a $230 \mathrm{~km}$ diameter spherical cap's gravity into harmonics (Farrell 1972) up to degree 200 converged to within $0.8 \%$ of that due to 24 support points.

Mascons spaced every $100 \mathrm{~km}$ are shown in figure 3.3a. Each mascon is assigned a mass equivalent to a layer of water $10 \mathrm{~cm}$ thick spread over the area of the mascon. The upward-continued synthetic accelerations implied by this mascon field are shown in figure 3.3e. Notice that the pattern is smooth, but the lack of mascons south of $50^{\circ} \mathrm{N}$ introduces a latitude dependence. Mascons spaced every $1050 \mathrm{~km}$ are shown in figure 3.3b. Again, each mascon is assigned a mass equivalent to a layer of water $10 \mathrm{~cm}$ thick spread over the area of the mascon. The upward-continued synthetic accelerations implied by this mascon field are shown in figure 3.3f. Errors due to the point mass approximation are prominent. Mascons spaced every $1050 \mathrm{~km}$ are shown in figure 3.3c. Again, each mascon is assigned a mass equivalent to a layer of water $10 \mathrm{~cm}$ thick spread over the area of the mascon. However, this time each mascon has a set of 8 support points which represent this layer of water more uniformly. The upward-continued synthetic accelerations implied by this mascon field are shown in figure 3.3g. Finally, mascons spaced every $1050 \mathrm{~km}$ are shown in figure $3.3 \mathrm{~d}$, each with 24 support points to help represent the $10 \mathrm{~cm}$ layer of water. The upward-continued synthetic accelerations implied by this mascon field are shown in figure $3.3 \mathrm{~g}$. Notice that the accelerations in $3.3 \mathrm{~g}$ are very similar to those in $3.3 \mathrm{a}$. 

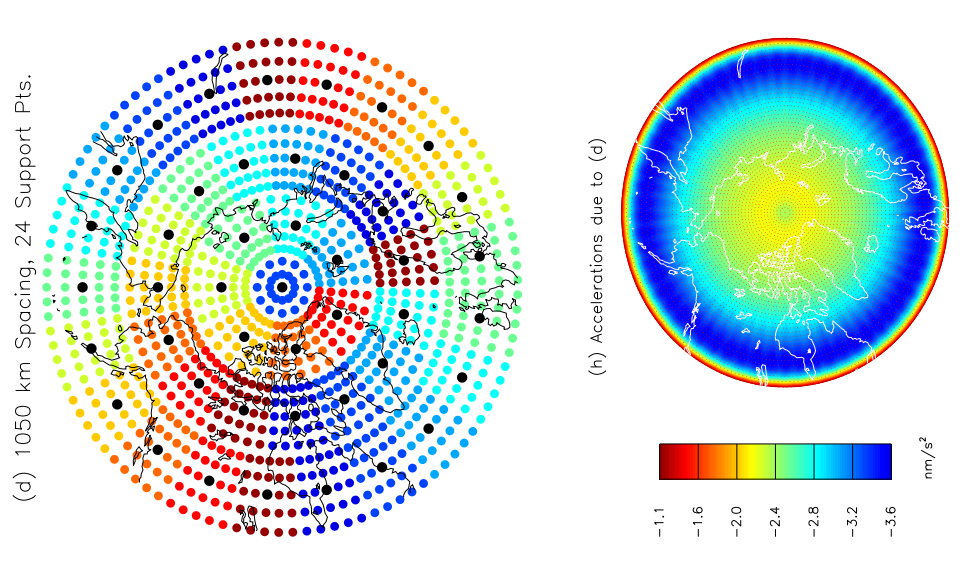

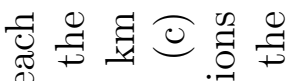

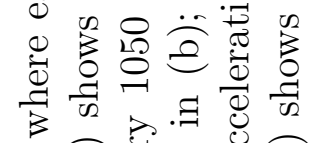

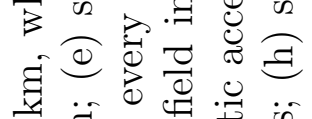

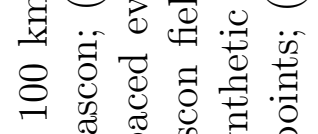

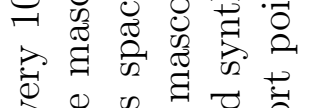

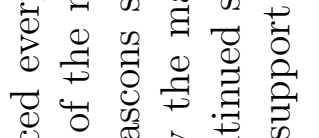
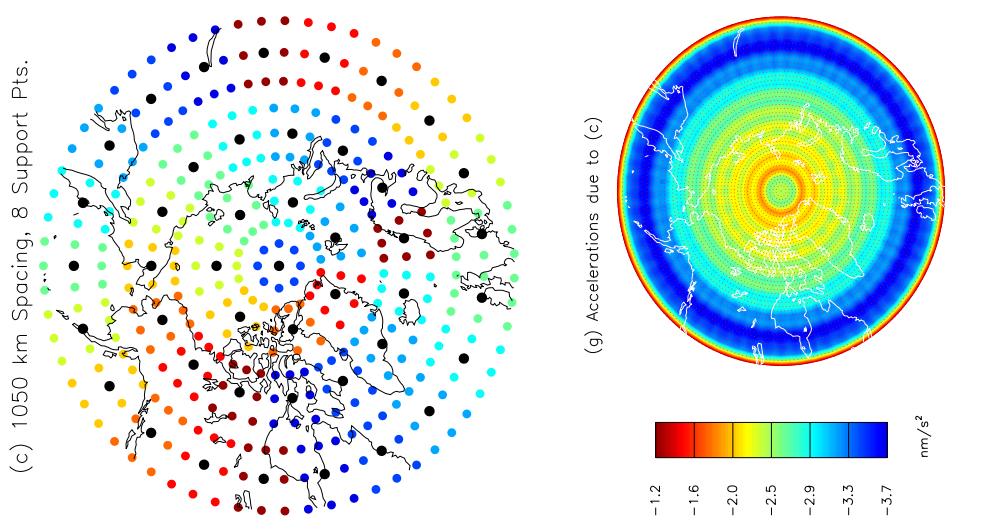

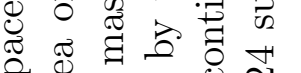

की

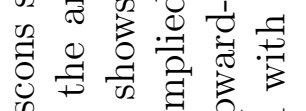

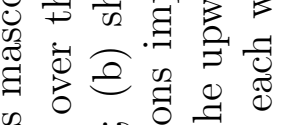

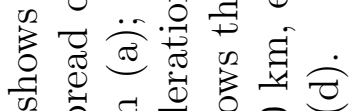

๙

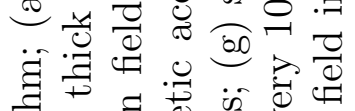
言苛 $\begin{aligned} & 0 \\ & 0\end{aligned}$

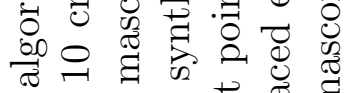
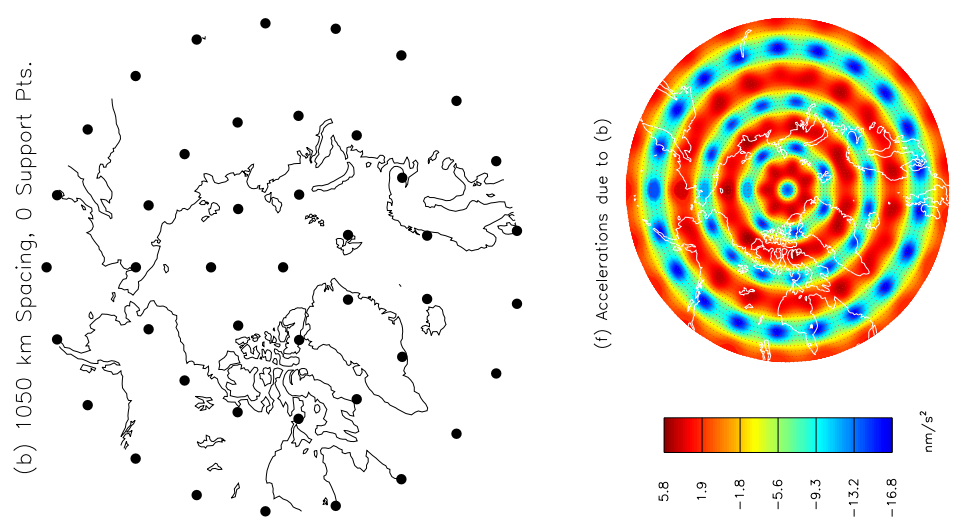

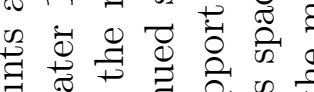

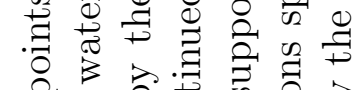
눔 Бे

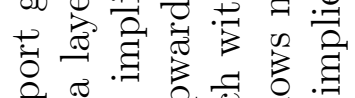
范

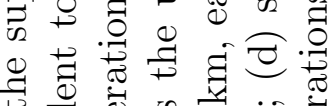

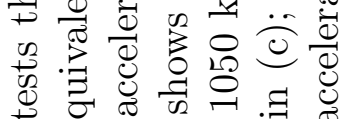
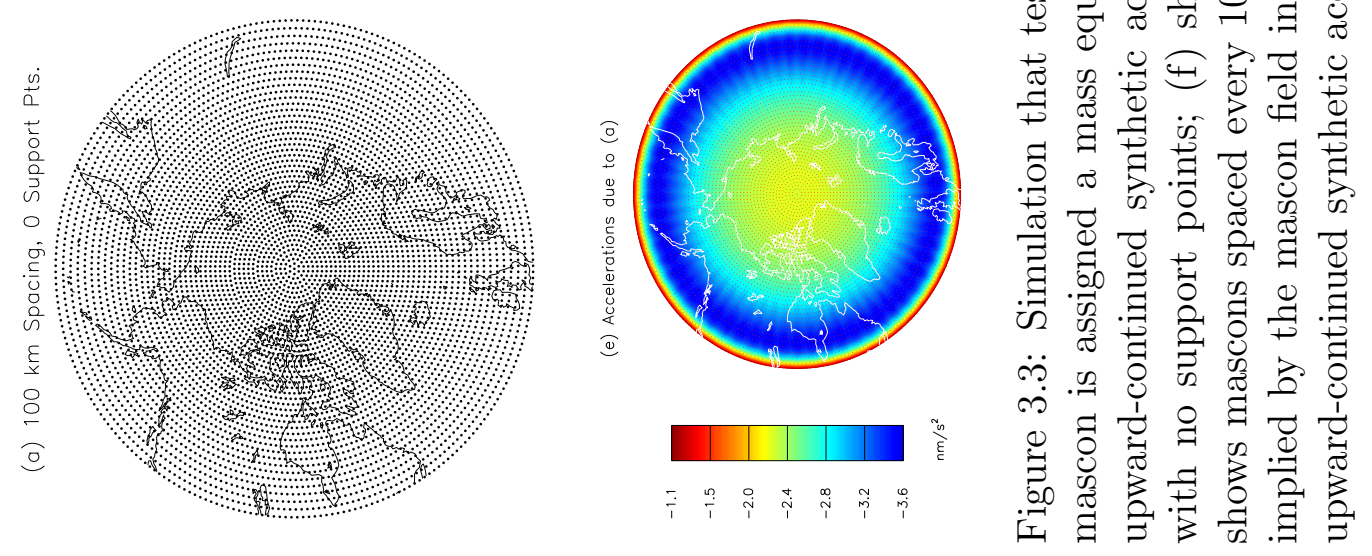


\subsection{Overlapping Regions}

The covariance matrix A for a global inversion would ordinarily fit into the computer's finite memory only when using a large grid spacing or solving for a small number of simultaneous temporal parameters. Instead, the grid is divided into regions which are inverted independently. Mascon amplitudes in each region are obtained by inverting only the accelerations recorded when the satellites are over that region. This introduces the problem of boundary effects; mass variations near region boundaries influence accelerations recorded in both regions.

To minimize noise induced by boundary effects, the regions are split into two distinct parts. Each region has a central part which is directly adjacent to other regions' central parts. Each region also includes an overlap around this central part; accelerations from the central and overlap parts are inverted to solve for mascon amplitudes in both the central and overlap parts. The mascon amplitudes in the overlap parts are then discarded, and mascon amplitudes from each region's central part are combined to form the final map.

In figures 3.4a,b, the central (overlap) parts of two regions are shown in red (green), and points outside the region are shown in blue. The central parts of each region are shown in figure 3.4c. Boundary effects are quantified by upward-continuing a $10 \mathrm{~cm} /$ year secular trend over land, then subtracting an inversion of these accelerations that uses these regions from an inversion that solves for the trend everywhere simultaneously. The resulting error for an overlap distance of $2050 \mathrm{~km}$ is shown in figure $3.4 \mathrm{~d}$; it decreases as the overlap distance increases. 
(a) Region \#1 of 6

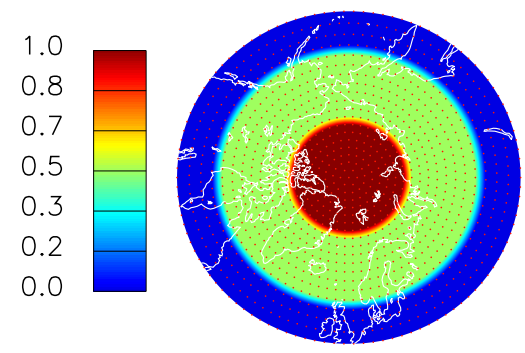

(c) All Regions' Central Parts

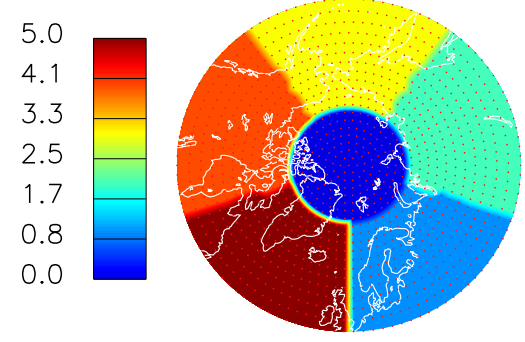

(b) Region \#2 of 6

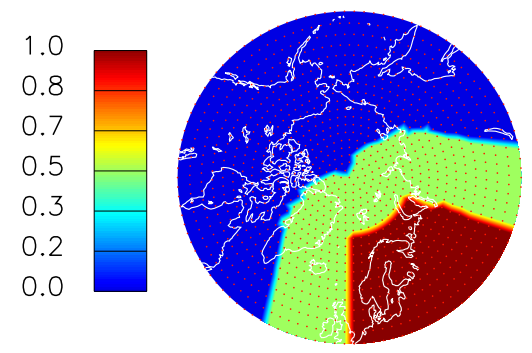

(d) Regions-Induced Error

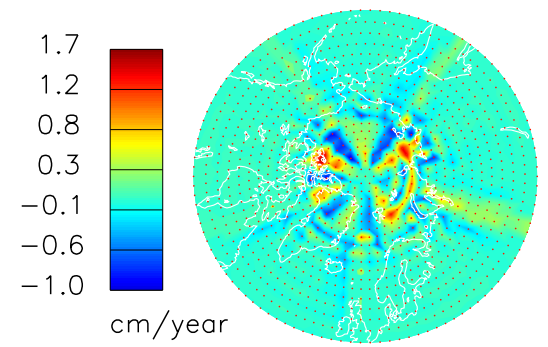

Figure 3.4: Overlapping regions; (a) and (b) display the central (overlap) parts of regions in red (green) and points outside the regions entirely as blue; (c) shows the central parts of all regions; (d) is the result of upward-continuing a $10 \mathrm{~cm} /$ year trend over land, then subtracting an inversion of these accelerations that uses these regions from an inversion that solves for the trend everywhere simultaneously. 


\section{Chapter 4}

\section{Simulations}

To test the inversion algorithm, synthetic mascon fields are created for two separate simulations. The centers of these mascons (indicated by red dots in figure 4.1) are separated by about $230 \mathrm{~km}$ and cover the Earth north of $50^{\circ} \mathrm{N}$ latitude. Each set of simulated mascon fields includes a $10 \mathrm{~cm} /$ year trend over land mascons (not shown) with no trend over ocean mascons, in addition to the $M_{2}$ and $K_{1}$ sine and cosine patterns shown in figure 4.1. In this context, "cosine" means the coefficient of $\cos (\omega t)$ where $t=0$ at J2000. These are upward-continued using Newtonian gravity to calculate the relative accelerations caused by those mascon fields as measured by GRACE. The actual positions of the GRACE satellites over seven years are used in this upward continuation process. The resulting synthetic accelerations are then inverted using the regularized least squares fit procedure described in chapter 3 to simultaneously deduce the implied surface mascon time dependencies for the secular trend as well as the $M_{2}$ and $K_{1}$ sine and cosine coefficients. This test is designed to assess the effects of regularization, and nothing else.

In both simulations, the input $M_{2}$ and $K_{1}$ sine maps are identical and both input cosine maps are zero. Output sine maps for $M_{2}$ and $K_{1}$ in both simulations are so similar that only the maps for $M_{2}$ are shown. They demonstrate that regularization doesn't appreciably attenuate continental-scale signals. Since cosine inputs are zero, the cosine solutions are presumed to be leakage errors from other inputs. The $K_{1}$ cosine map errors are $\sim 15 \%$ of the maximum input sine amplitude, largest at the north pole and $\sim 20$ times larger than 

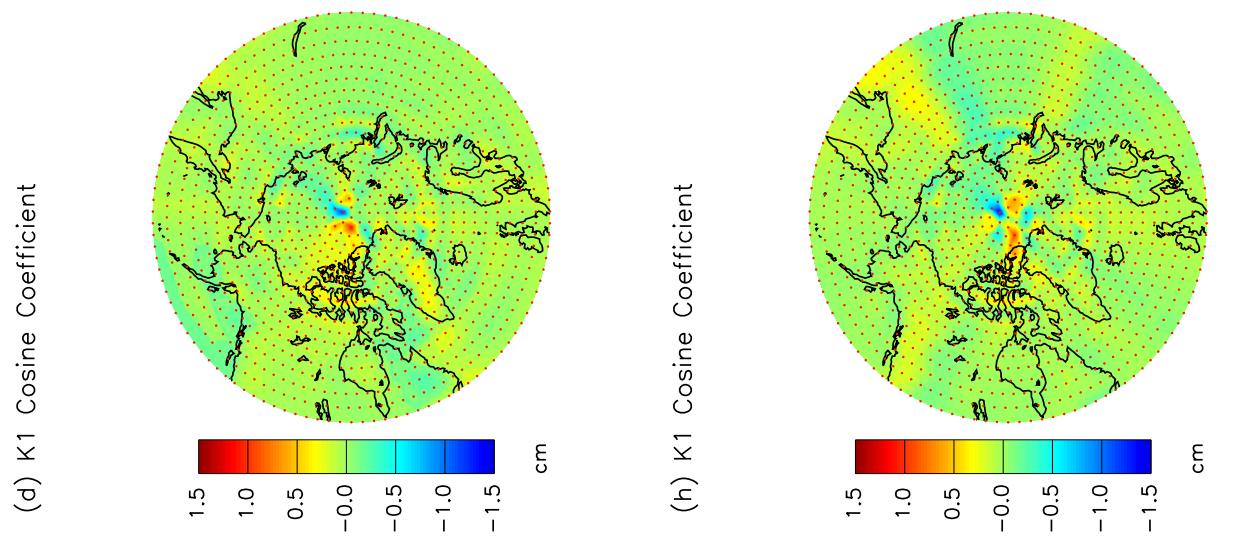

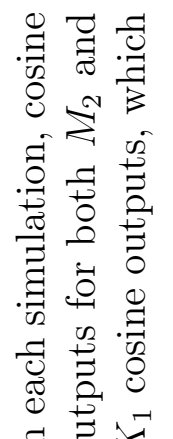
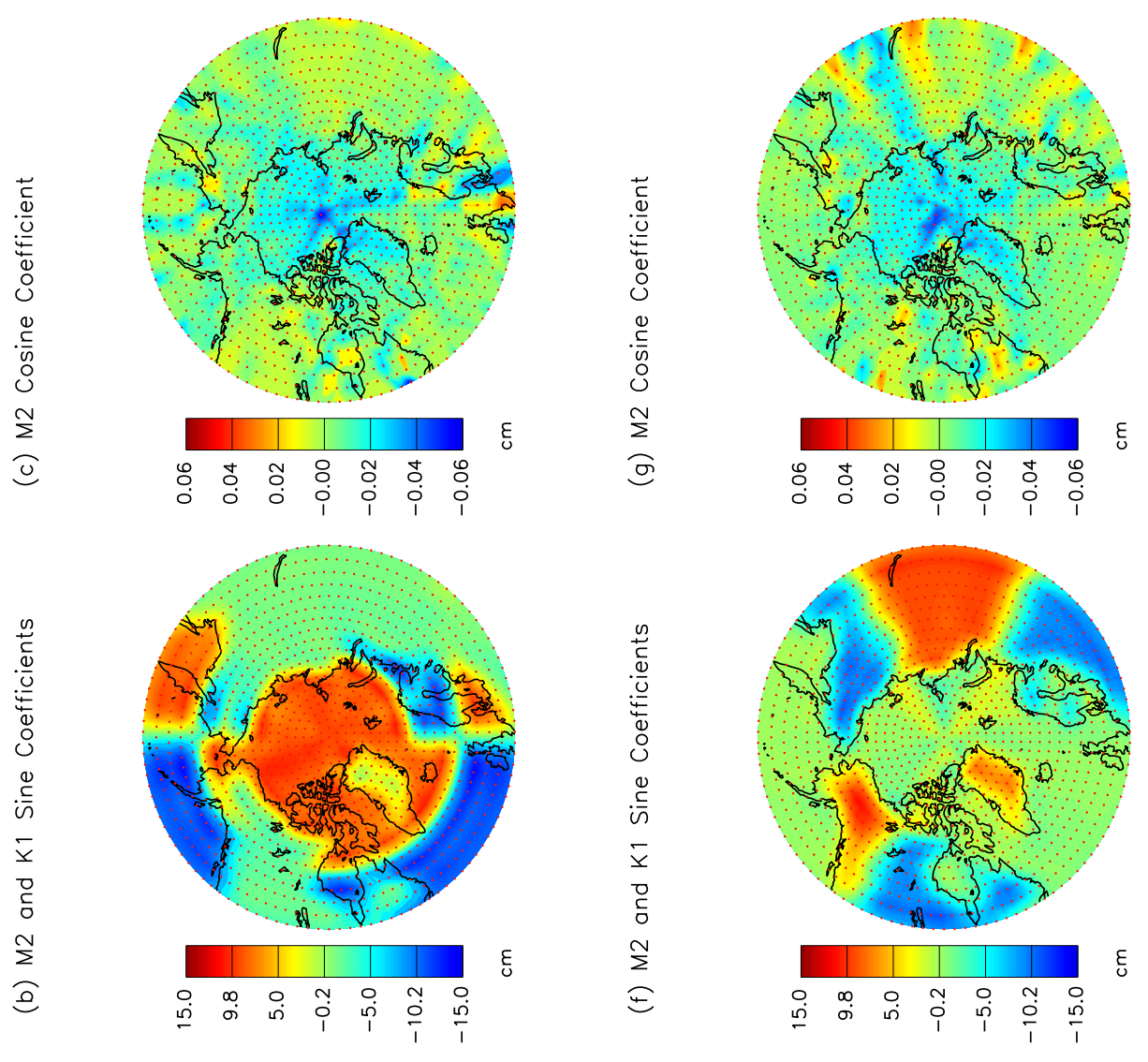

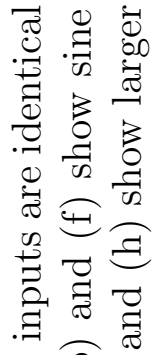

를

콜

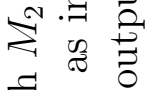

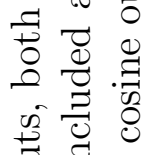
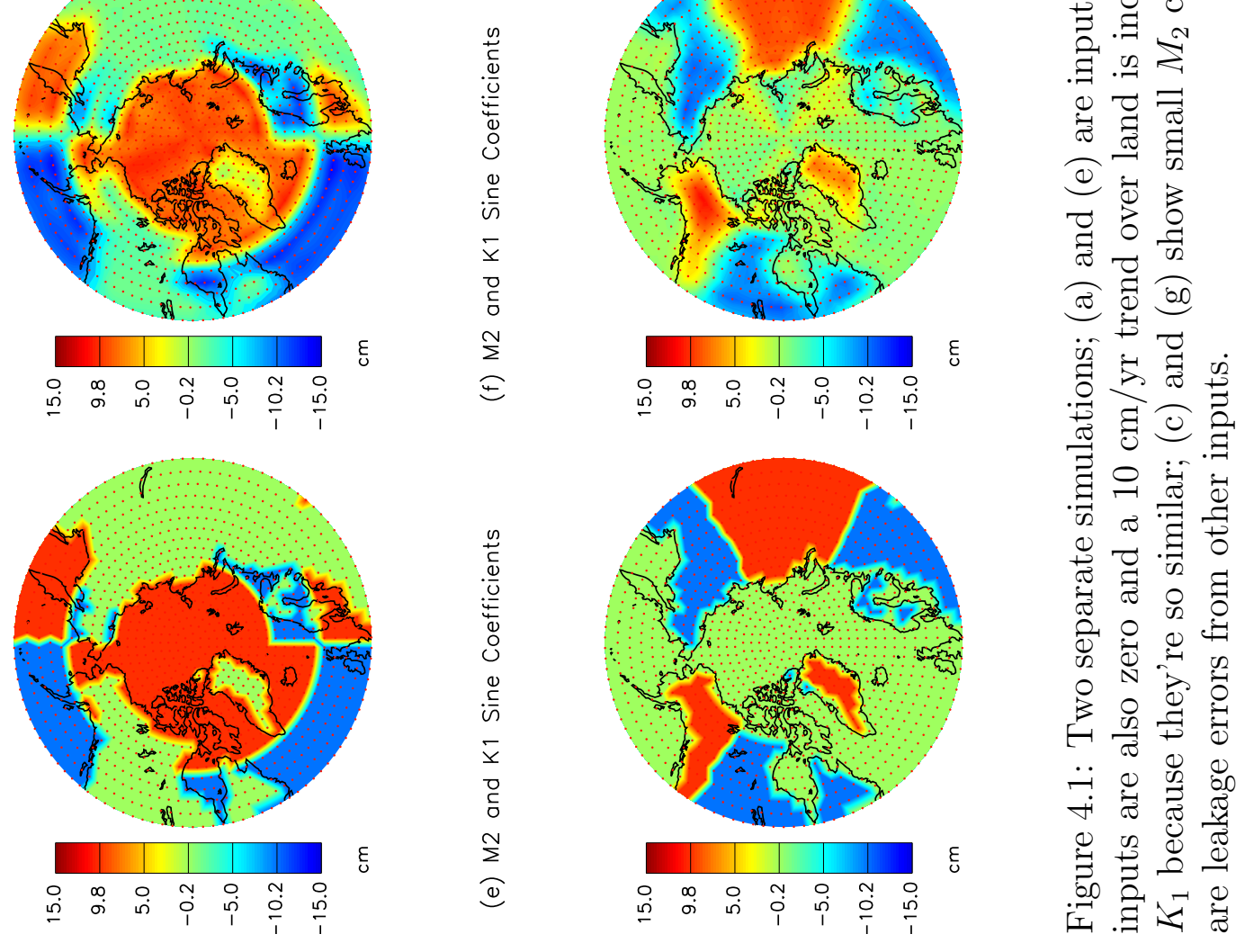
errors in $M_{2}$. When the secular trend is removed from the input, the errors in $K_{1}$ are no longer significantly larger than those in $M_{2}$, suggesting that the simulated $K_{1}$ north pole anomaly results from interactions between $K_{1}$ and the trend when regularization is applied. In these simulations, the Newtonian gravity algorithm is applied twice, once for upward continuation from the input mascon fields to the synthetic acceleration time series, and once for the inversion from the acceleration time series to the output mascon fields. Instrumentation noise isn't included in the simulated data, but the same regularization used with noisy real data is applied.

The resolution is determined by inverting the accelerations due to a single simulated non-zero mascon shown in figure 2.1. The resulting mascon field blurs the single point into a broader feature with a half-width of $\sim 300 \mathrm{~km}$ and attenuates the peak amplitude by a factor of four. The mascon spacing of $\sim 230 \mathrm{~km}$ is chosen because it's small enough not to constrain the resolution but large enough so the resulting covariance matrix fits into the computer's memory. 
(a) Input Mascon Field

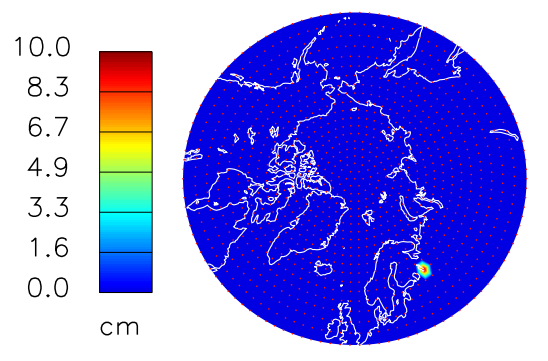

(c) Output Mascon Field
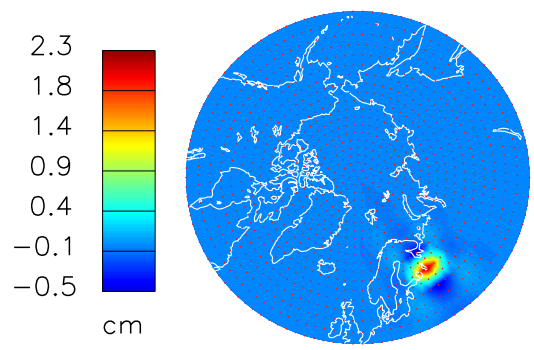

(b) Simulated Accelerations
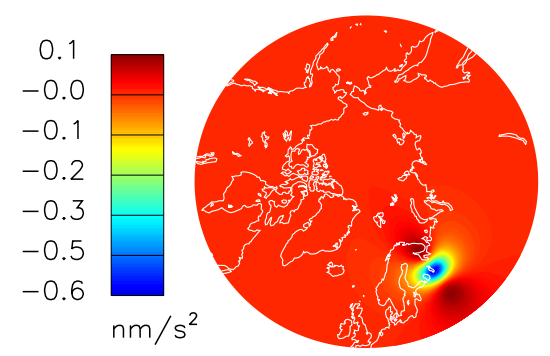

Figure 4.2: Simulation to test resolution; (a) is the input mascon field; (b) display the simulated accelerations resulting from upwardcontinuing the input mascon field; (c) is the inversion of those simulated accelerations. 


\section{Chapter 5}

\section{Real Data Inversions}

Real acceleration data generated at the Jet Propulsion Laboratory (JPL) are inverted using the regularized least squares fit procedure described in chapter 3 to produce mascon fields for tides $M_{2}$ and $K_{1}$ in figure 5.2. All parameters are inverted simultaneously over the entire globe north of $50^{\circ} \mathrm{N}$, including the non-tidal parameters shown in figure 5.1 and $O_{1}$ and $P_{1}$ in figure 5.3.

The inverted trend in figure $5.1 \mathrm{~b}$ reproduces the well-known mass loss on Greenland's southeastern coast; calculating the trend using GRACE datasets with different endpoints confirms that this mass loss spread up the northwestern coast starting around late 2005 (Khan et al. 2010). The positive trends in Canada and Scandinavia are known to be caused by post-glacial rebound. The trends in the ocean around the north pole are unlikely to be geophysical in origin; they're likely due to the interaction between the trend and the $K_{1}$ north pole anomaly. The inverted constant term in figure 5.1a is highly correlated with the trend because the static gravity model removed from the GRACE data doesn't account for the trend since the start of the GRACE timeseries, and the reference epoch used is equal to the midpoint of the GRACE timeseries. Thus, the constant term describes the total change in the surface mass distribution from the start of the GRACE time series to its midpoint.

The inverted annual amplitude in figure 5.1d is dominated by the glaciers in Alaska, and displays a small anomaly at the north pole. The large $(\sim 10 \mathrm{~cm})$ annual amplitude in the East Siberian Sea isn't well understood. This feature is present in other inversions (Meister 
and Wingham 2010) and remains even after accounting for the motion of the Earth's center of mass relative to its geometric center (Swenson et al. 2008). Runoff from snowmelt likely disperses in the ocean so quickly that it probably wouldn't be detected as an annual cycle in the ocean. However, associated seasonal variations in wind-driven circulation patterns such as the Beaufort Gyre could be responsible for the large annual amplitude in the East Siberian Sea.

The inverted semi-annual amplitude in figure 5.1b is well-correlated with the annual amplitude because the annual cycle of snowfall and snowmelt is asymmetric (snow melts in the spring faster than it accumulates during the fall). This causes the annual cycle of surface mass distribution in the cryosphere to have significant power at the semi-annual period. The semi-annual amplitude is regarded as a non-tidal parameter here even though it matches the period of the long-period tide $S_{s a}$ (see table 1.3). That's because long-period tides have historically been modelled as though they are in equilibrium with the tidal potential, which Proudman (1960) shows is a good approximation for tides with periods greater than $\sim 50$ days. As a result, the FES2004 semi-annual tide $S_{\text {sa }}$ removed from the GRACE data is likely to be so accurate that the inverted semi-annual amplitude can be reasonably assumed to be dominated by non-tidal effects.

On the left of figure 5.2 are FES2004 $M_{2}$ and $K_{1}$ amplitudes; note that the FES2004 figures have color scales that are much larger than the other maps and saturated in the sense that white areas on the maps have larger amplitudes than the tops of their respective color scales. Corresponding mascon amplitude maps from the inversion of real data follow, with red dots indicating the centers of the mascons, and the average amplitude on land noted (see section 5.1). Next are maps showing the changes in FES2004's amplitudes when the inverted residual estimates are added to the original FES2004 model. On the right are amplitudes of the differences between two independent mascon solutions each using 3.5 years of independent data. Figure 5.3 is similar except it shows $O_{1}$ and $P_{1}$ from the same inversion. Figure 5.4 displays results from a separate inversion for $Q_{1}$ and $N_{2}$, which are much smaller 
(a) Constant Term
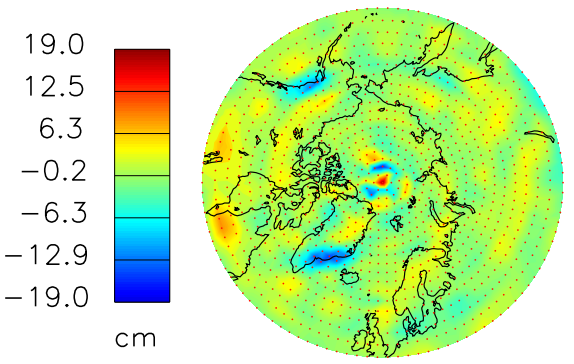

(c) Semi-Annual Amplitude
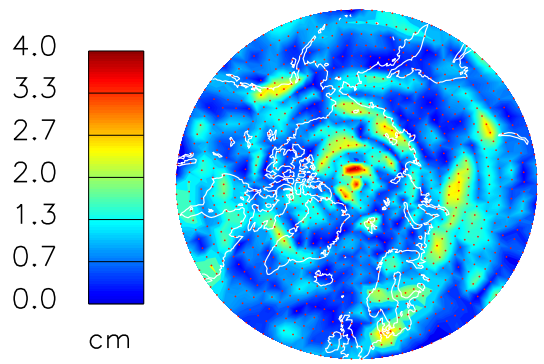

(b) Secular Trend

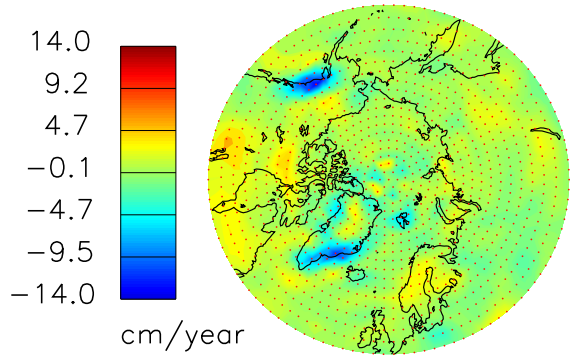

(d) Annual Amplitude

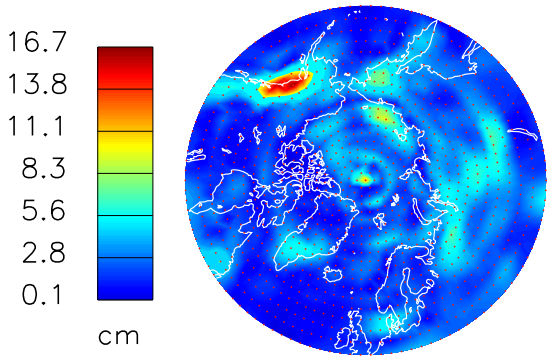

Figure 5.1: Inverted non-tidal parameters: (a) constant term; (b) secular trend; (c) semiannual amplitude; (d) annual amplitude. 
tides and therefore don't rise appreciably over their noise floors.

For deep ocean tides, FES2004 relies primarily on TOPEX/Poseidon altimetry data which don't extend north of $66^{\circ} \mathrm{N}$. One might expect that mascon solutions should reveal systematically larger residual tidal amplitudes north of $66^{\circ} \mathrm{N}$ versus south of $66^{\circ} \mathrm{N}$, which isn't the case for any examined tides. In the oceans north of $50^{\circ} \mathrm{N}$, the mascon solutions tend to reduce the FES2004 amplitudes for $M_{2}, K_{1}, O_{1}$, and $P_{1}$.

The GRACE-observed difference between FES2004 and its expansion to degree 90 (the maximum degree used when removing FES2004 from the raw GRACE data) is called truncation error. The difference between the land mask used in GRACE processing and the actual coastlines is called the land mask error.

Truncation errors are simulated by subtracting the $l_{\max }=90$ spherical harmonic expansion of FES2004 from the original FES2004 model. These truncation errors are upwardcontinued to GRACE accelerations, then inverted using the same procedure that is applied to the real accelerations. Degree 90 truncation errors for FES2004 $M_{2}$ and $K_{1}$ are shown in figure 5.5. Panel (a) is the FES2004 $M_{2}$ truncation error on the surface using $230 \mathrm{~km}$ mascons; (e) is the result of upward-continuing the map in (a) and inverting using $230 \mathrm{~km}$ mascons; (b) is the FES2004 $M_{2}$ truncation error on the surface using $100 \mathrm{~km}$ mascons; (f) is the result of upward-continuing the map in (b) and inverting using $230 \mathrm{~km}$ mascons; (c) is the FES2004 $K_{1}$ truncation error on the surface using $230 \mathrm{~km}$ mascons; (g) is the result of upward-continuing the map in (c) and inverting using $230 \mathrm{~km}$ mascons; (d) is the FES2004 $K_{1}$ truncation error on the surface using $100 \mathrm{~km}$ mascons; (h) is the result of upward-continuing the map in (b) and inverting using $230 \mathrm{~km}$ mascons. Paradoxically, using $100 \mathrm{~km}$ mascons increases the maximum amplitude on the surface compared to using 230 $\mathrm{km}$ mascons, but drastically reduces the inverted amplitude after upward-continuing and inverting the accelerations due to those mascons. This is less mysterious than it may appear, because GRACE is sensitive to mass (not water thickness) and the $100 \mathrm{~km}$ mascons are $\sim 2.3^{2}$ times smaller than the $230 \mathrm{~km}$ mascons. Also, the degree 90 truncation errors 

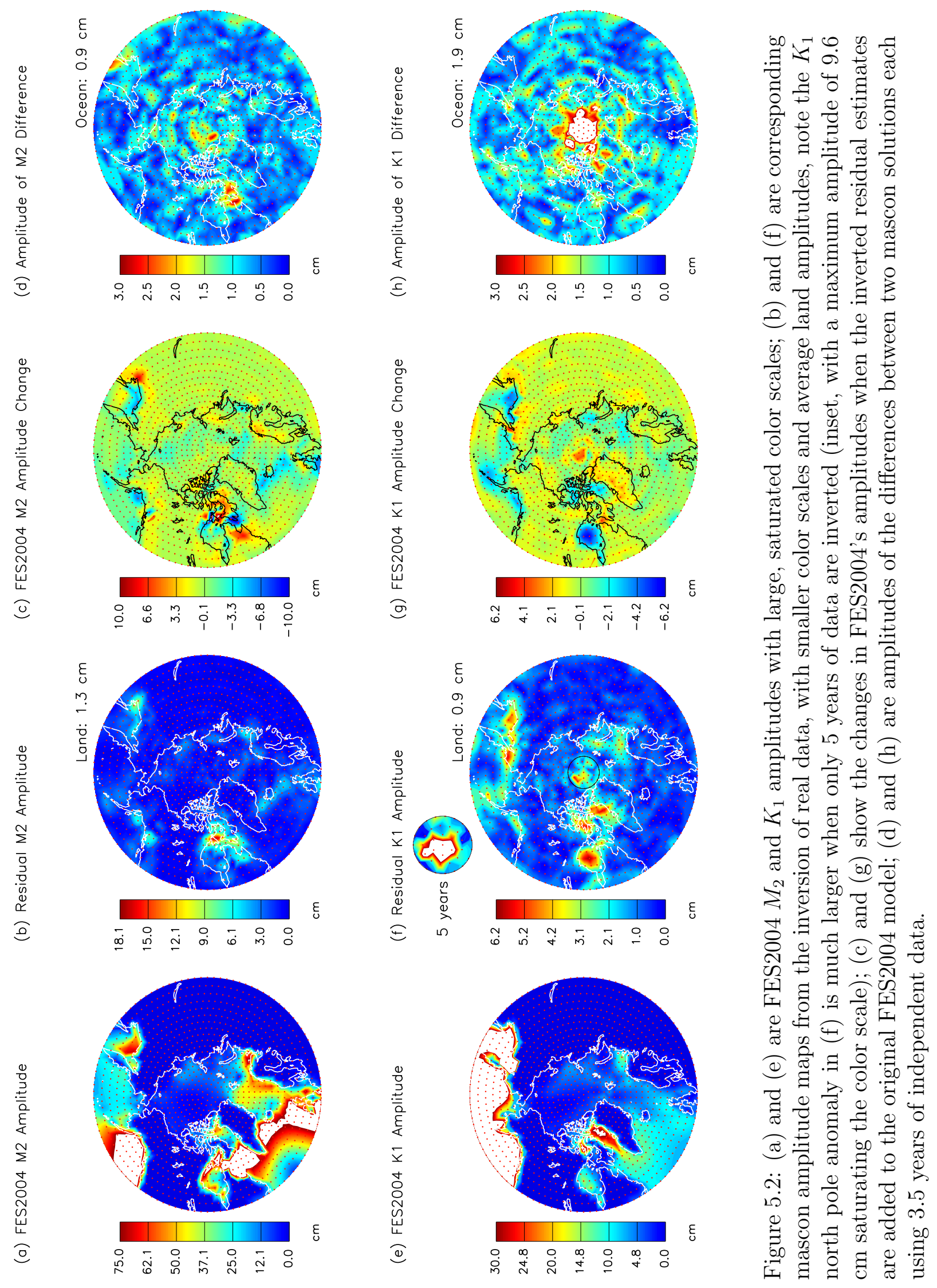

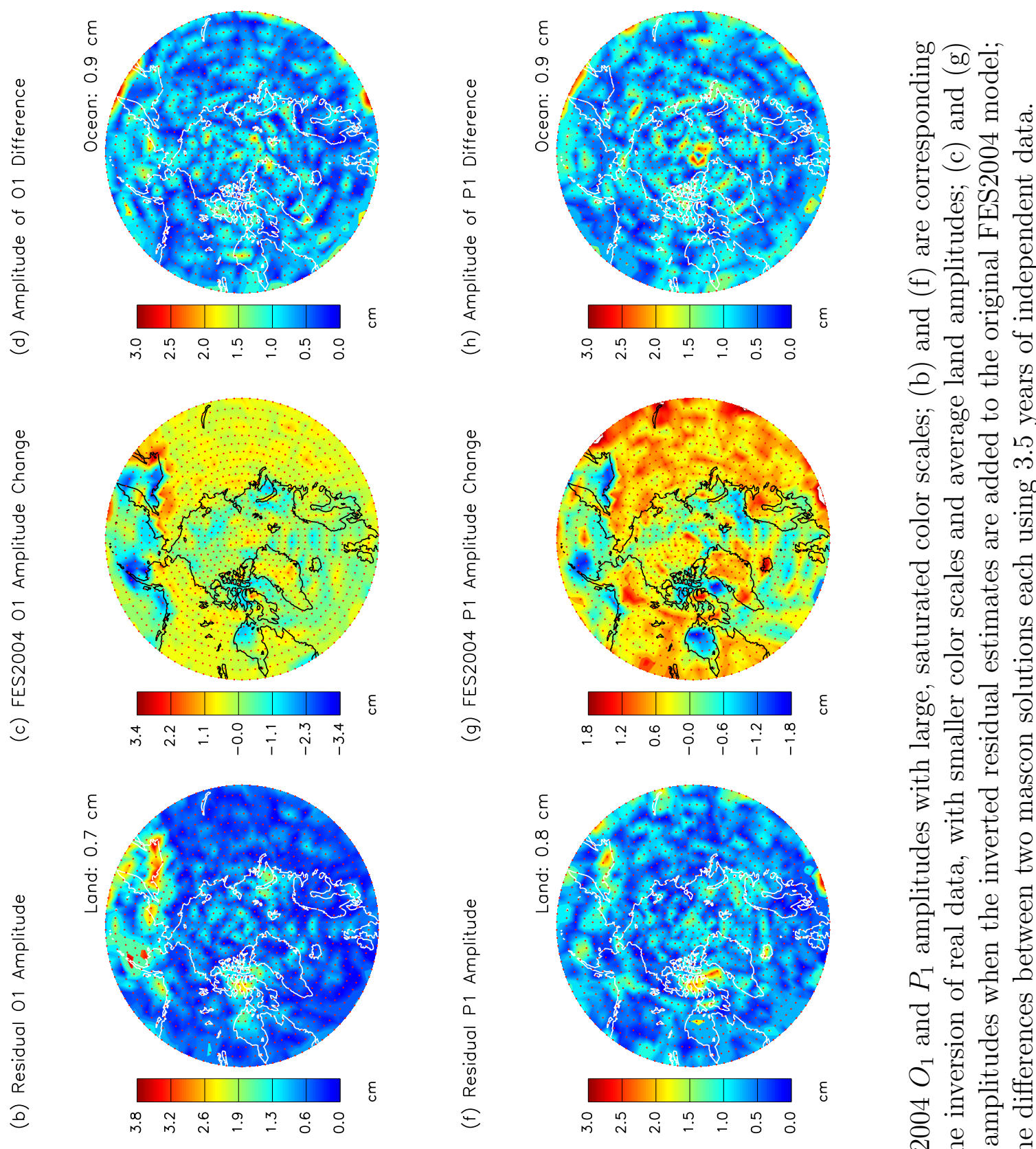

อิ

if 800

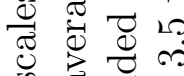

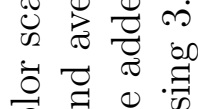

苛

D

志芯泀

\&

元 $\bar{\sigma}$

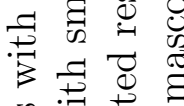

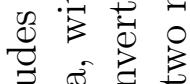

西要

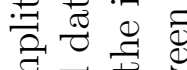

สี

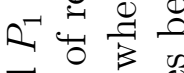

चี

त. $\frac{0}{\sigma}$

○岂: 离

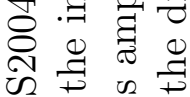
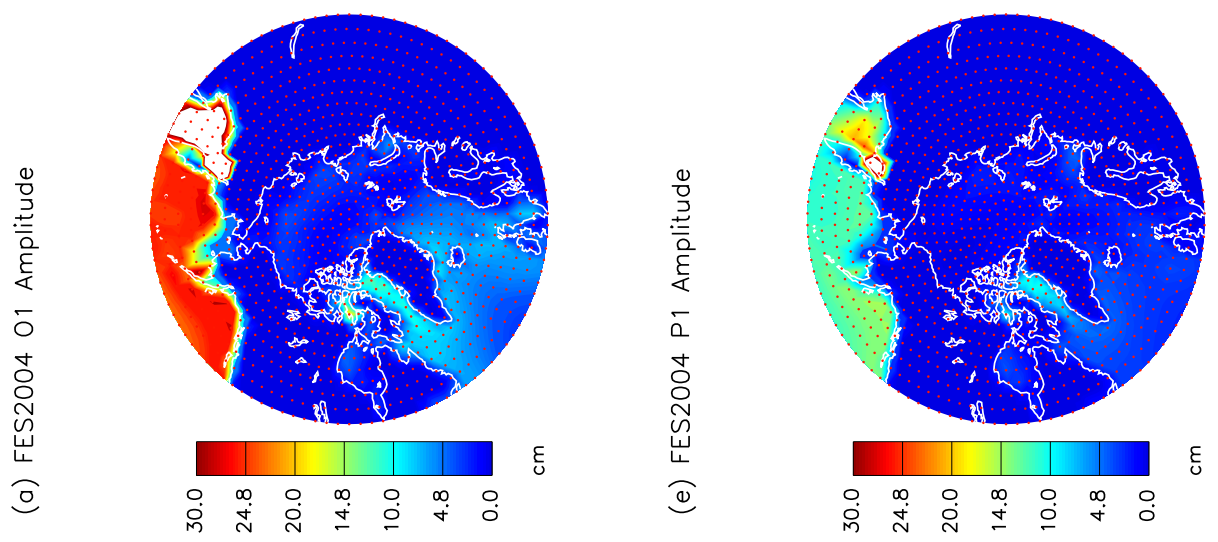

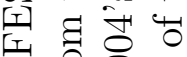

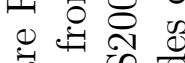

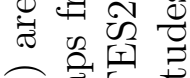

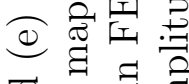

चี

తి

๑. द्वु

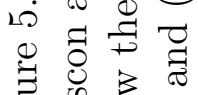

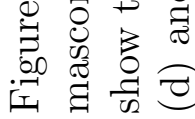



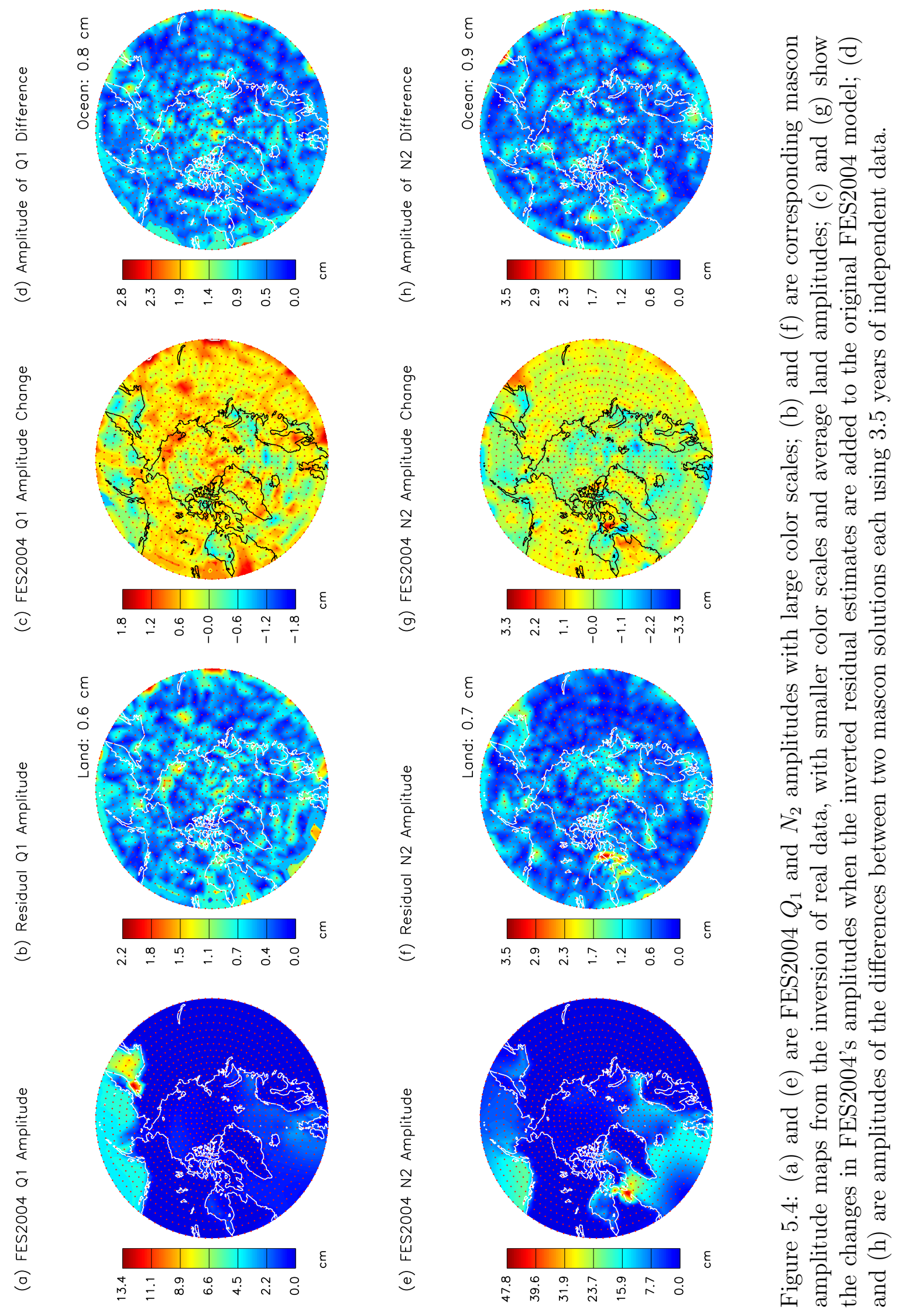
only have short-scale features which GRACE can't resolve. The inverted truncation errors for $M_{2}$ and $K_{1}$ are well below the $\sim 1 \mathrm{~cm}$ GRACE noise floor determined in section 5.1.

Because tide models give sea surface height but GRACE senses mass, the GRACE tide correction involves a summation over all non-zero FES2004 grid points. Thus, it's important that FES2004 give sea surface height everywhere in the ocean and does not give a non-zero sea surface height anywhere on land. Errors in the FES2004 land mask could play a larger role in narrow inlets such as Hudson Strait and the Canadian archipelago, but they should also produce relative errors of similar magnitude for different tides in the same location. This correlation isn't observed; for instance the $K_{1}$ residual features in the Canadian archipelago and Baffin Bay don't have corresponding $M_{2}$ residual features in the same places, even though the FES2004 amplitudes for both tides are appreciable in those locations.

Figure 5.6 displays the long-period tides $M_{f}$ and $M_{m}$ from a separate inversion using 5 years of GRACE data. The inverted $M_{f}$ residual estimate is notable because the amplitude north of Hudson Bay is $\sim 6$ times larger than the original FES2004 amplitude in that location, which is the largest relative correction for any examined tide. The fact that $M_{f}$ displays larger relative corrections than $M_{m}$ is consistent with the expectation (Proudman 1960) that $M_{f}$ 's period is too short for frictional effects to damp away deviations from the equilibrium response.

\subsection{Uncertainty Estimates}

The mascon fields from the inversions tend to have much larger tidal amplitudes in the ocean than on land. Land tides at mascons that don't intersect the ocean (colored red in figure 3.1a) are assumed to be due to some combination of truncation errors in the expansion of FES2004, errors in body tide modelling, processing and instrumentation errors in the GRACE data, aliasing, leakage from short-period non-tidal ocean signals, aliased hydrologic/cryospheric processes, and errors in the ocean loading models removed from the raw data. These land tide solutions can be used to determine error estimates for the ocean 

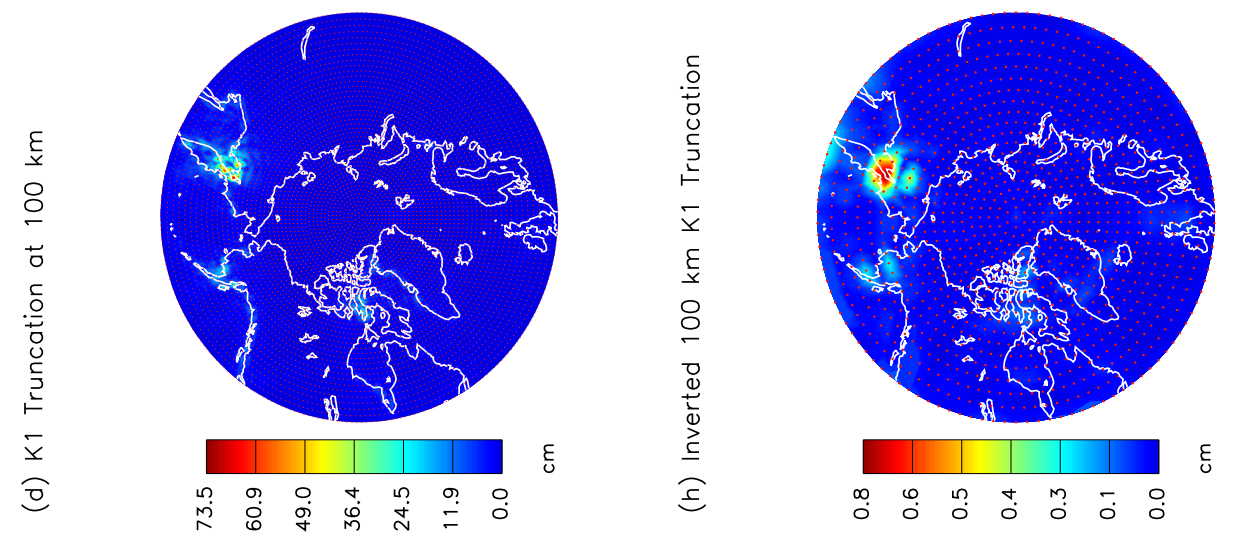

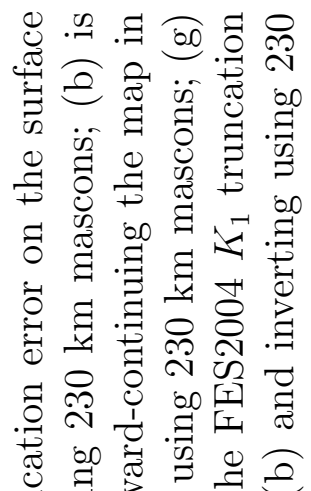

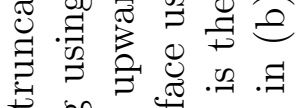
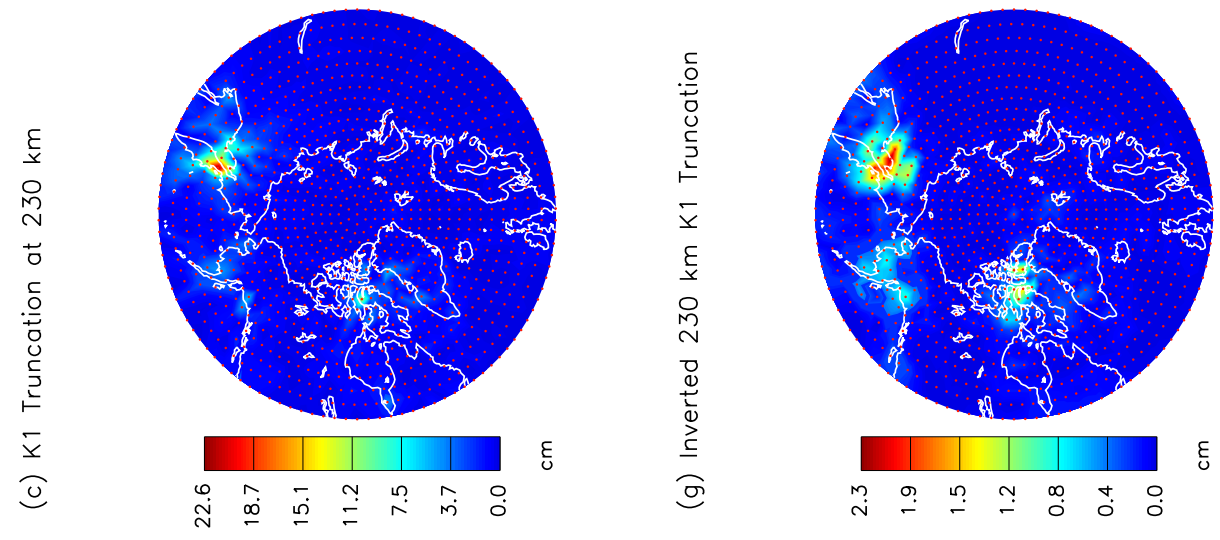

光

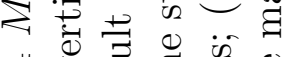

ஓं

๙ิ

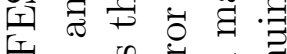

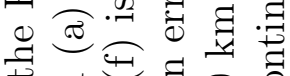

四

(ఠ)

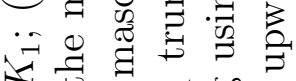

임
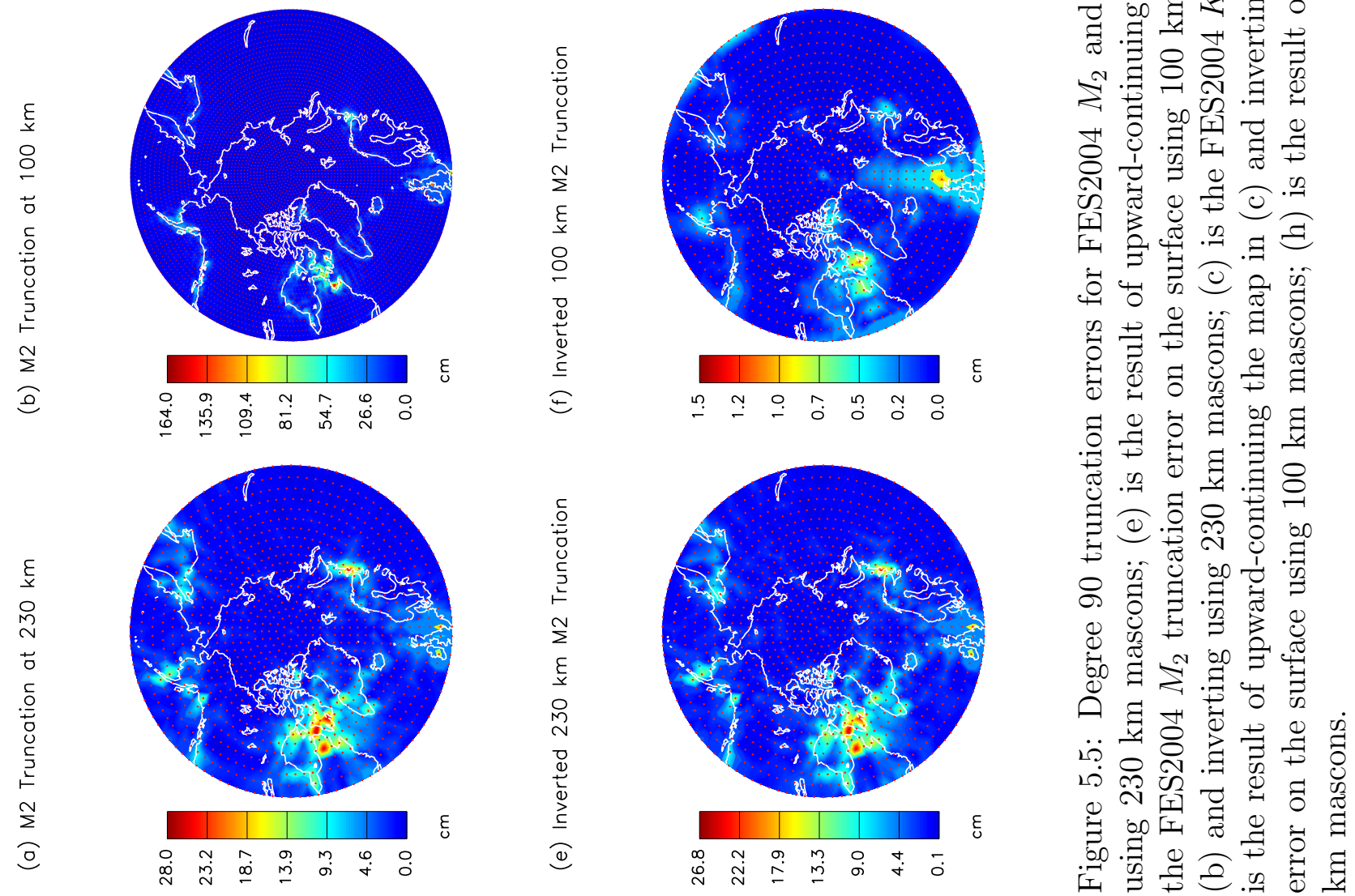

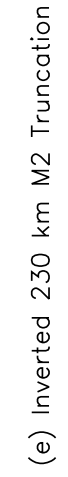


(a) FES2004 Mf Amplitude

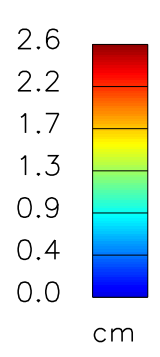

(c) FES2004 Mm Amplitude
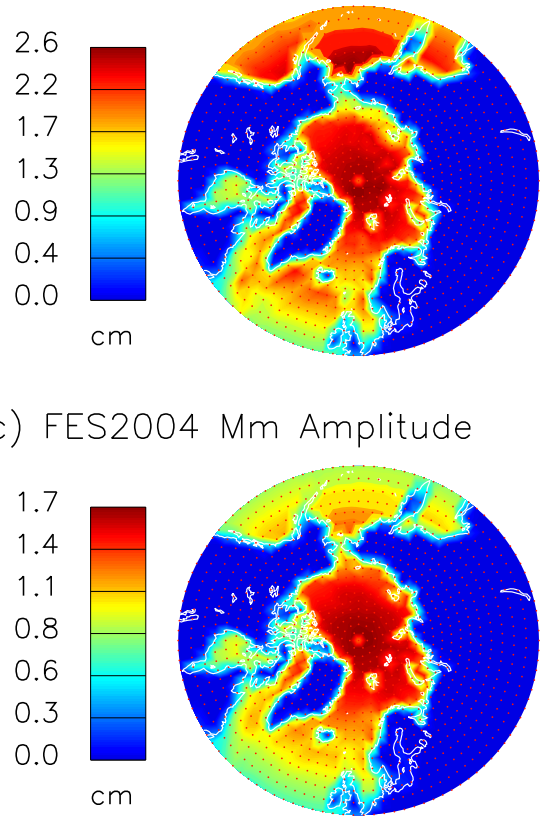

(b) Mf Amplitude

Land: $0.8 \mathrm{~cm}$
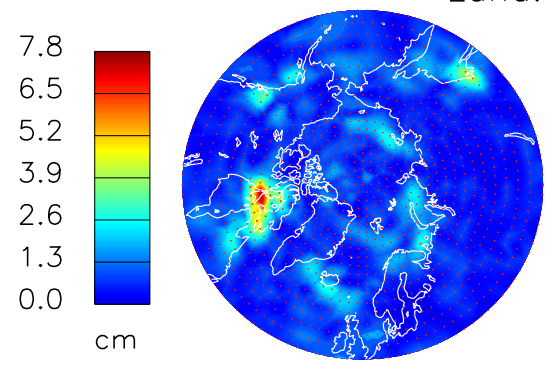

(d) Mm Amplitude

Figure 5.6: (a) and (c) are FES2004 $M_{f}$ and $M_{m}$ amplitudes; (b) and (d) are corresponding mascon amplitude maps from the inversion of real data, with average land amplitudes. 
tide solutions by defining the true tidal signal and the observed tidal estimate at a given point on the Earth's surface

$$
\begin{gathered}
T_{\text {true }}(t)=(a) \cos (\omega t)+(b) \sin (\omega t) \\
T_{\text {obs }}(t)=(a+\delta a) \cos (\omega t)+(b+\delta b) \sin (\omega t) .
\end{gathered}
$$

The corresponding true and observed tidal amplitudes are

$$
\begin{gathered}
A_{\text {true }}^{2}=a^{2}+b^{2} \\
A_{o b s}^{2}=(a+\delta a)^{2}+(b+\delta b)^{2} .
\end{gathered}
$$

Given N GRACE time series of length 7 years, and assuming the errors $\delta a$ and $\delta b$ are drawn from a Gaussian distribution with zero mean (and so are uncorrelated with the true signal and with each other), implies a statistical mean observed squared amplitude of

$$
\left(A_{\text {obs }}^{2}\right)_{\text {mean }}=A_{\text {true }}^{2}+\operatorname{var}(\delta a)+\operatorname{var}(\delta b) .
$$

Note that the statistical mean observed squared amplitude is always biased positively even by errors with zero mean due to the nonlinear relationship between the amplitude and the cosine/sine coefficients. $A_{\text {true }}$ should be zero on land, so the average value of $A_{\text {obs }}$ on land is an estimate of $\sqrt{\operatorname{var}(\delta a)+\operatorname{var}(\delta b)}$. Assuming that land and ocean mascons are affected by similar noise processes, and thus have similar values of $\sqrt{\operatorname{var}(\delta a)+\operatorname{var}(\delta b)}$, any ocean mascons with $A_{o b s} \leq \sqrt{\operatorname{var}(\delta a)+\operatorname{var}(\delta b)}$ are unlikely to be good representations of $A_{t r u e}$. Each inverted tidal amplitude's average land tide value is noted in figure 5.2, and can serve as a rough error estimate for the tidal solution over the ocean. 
A second uncertainty estimate is derived from the difference between two independent solutions, each using 3.5 years of data. This estimate has the spatial pattern shown in figures $5.2 \mathrm{~d}$,h along with an ocean average to compare with the previously described land tide average. The ocean tide difference using 3.5 years of data likely overestimates the uncertainty in the 7 year solution. This is especially true for $K_{1}$ at the north pole (see section 5.4), and when the north pole anomaly is removed from figure $5.2 \mathrm{~h}$, the average $K_{1}$ ocean difference drops from $1.9 \mathrm{~cm}$ to $\sim 1.2 \mathrm{~cm}$. These estimates both support a $\sim 1 \mathrm{~cm}$ uncertainty, though the difference maps reach $2-3 \mathrm{~cm}$ in some places. Estimating uncertainty using two independent solutions doesn't assume that oceanic noise is equal to or smaller than land noise, unlike the uncertainty estimate derived from land mascons.

\subsection{Noise Reduction}

The accelerations implied by the mascon fields are guaranteed to reduce the variance of GRACE accelerations used in the inversion. Some GRACE accelerations are withheld from another inversion for a more independent test of the accuracy of the mascon fields. A second acceleration time series with approximately five years of data (8 April 2002 to 3 June 2007 ) is used in this section because it was filtered with a 50 second cutoff period, and is thus less noisy than the 20 second dataset. The first $80 \%$ of the accelerations are used to solve for tides and a non-tidal component consisting of a constant, trend, and semi-annual and annual amplitudes. The last $20 \%$ of the data are reserved to verify that the inverted mascons reduce the variance of acceleration values not used in the solution.

The accelerations due to the non-tidal part of the solution are subtracted from the original accelerations, then the additional reduction in variance due to the tides is calculated. The reduction due to tides is calculated separately for ocean tides (defined as tides at mascons with at least some ocean) and land tides (tides at mascons with no ocean - see figure 3.1a). Each variance reduction in table 5.1 is calculated separately for accelerations used in the inversion and those reserved for verifications. 
Table 5.1: Variance reduction due to tides; surface sums are computed separately for ocean and land mascons by summing the squares of all the tidal cosine and sine coefficients in $\mathrm{kg}$ on the Earth's surface; these residual tidal estimates reduce the variance of GRACE accelerations used in the inversion, and accelerations reserved for verification; the variance reduction due to land tides decreases more than ocean tides when comparing the variance reduction of verification accelerations to accelerations used in the inversion.

\begin{tabular}{lllll}
\hline & & \multicolumn{3}{c}{ Accelerations Variance Reduction } \\
Tide & Surface Sum $\left(\mathrm{kg}^{2}\right)$ & Inversion (\%) & Verification (\%) & Decrease (\%) \\
\hline Ocean & $2.22 \times 10^{27}$ & 1.76 & 1.33 & 75.7 \\
\hline Land & $3.99 \times 10^{26}$ & 0.35 & 0.18 & 51.2 \\
\hline
\end{tabular}

In 100 Monte Carlo tests, the accelerations due to the ocean tides are subtracted from a random time series of accelerations with mean and variance equal to the GRACE verification accelerations after the non-tidal accelerations have been subtracted. The ocean tides never reduce the variance but always increase it by at least 1.1\%. For comparison, table 5.1 shows that ocean tides reduce the verification GRACE variance (after non-tidal subtraction) by $1.3 \%$, corresponding to an RMS reduction of $0.03 \mathrm{~nm} / \mathrm{s}^{2}$.

The ocean tides reduce the variance of the verification accelerations by $76 \%$ as much as they reduce the variance of inverted accelerations. The land tides, however, reduce the variance of the verification accelerations by $51 \%$ as much as they reduce the variance of inverted accelerations. This suggests that land tides are noisier than ocean tides, but also shows that land tides aren't entirely random (otherwise they wouldn't reduce the variance at all.) Thus, roughly $51 \%$ (by variance) of the land tide estimate may represent mis-modelled ocean loading, mis-modelled body tides, or aliased hydrological signals which would also appear in the verification accelerations.

\subsection{Power Spectra}

A second acceleration time series, filtered with a 50 second cutoff period, is sent through a Fast Fourier Transform to obtain the power spectra in figure 5.7a. Accelerations used in the inversion and those reserved for verification both yield spectra with one sharp peak at 
$0.003 \mathrm{~Hz}$ and a second broader peak centered at $0.02 \mathrm{~Hz}$. The second peak has a period of 50 seconds because that's the period at which the CRN filter starts to remove accelerometer (and other instrumentation) noise. The verification time series has more power than the inversion time series only when the last $20 \%$ of the data are used for verification; this difference isn't reproduced when any other segment of equal size is used for verification.

A synthetic spectrum using ten tides in FES2004 $\left(M_{2}, K_{1}, K_{2}, O_{1}, P_{1}, S_{2}, N_{2}, Q_{1}\right.$, $\left.M_{f}, M_{m}\right)$ is shown in figure $5.7 \mathrm{~b}$. The synthetic and real noise spectra have the first peak in common, but the lack of a second peak in the synthetic spectrum suggests that power at those frequencies in the real data spectrum probably isn't caused by tides. Note that these power spectra are more closely related to the spatial pattern of the tides than to their tidal periods because the GRACE orbital period of $\sim 90$ minutes is much shorter than even semi-diurnal tidal periods. The real data inversions in chapter 5 are therefore presumably attempting to fit the signal comprising the first peak. To test this, the real data inversions are upward continued and subtracted from the original acceleration time series. The relative variance reductions due to the mascons are shown in figures $5.7 \mathrm{c}, \mathrm{d}$.

Figures 5.7c,d suggest that $\sim 90 \%$ of the noise reduction in the first peak can be attributed to the non-tidal parameters. The remaining noise reduction is due to ocean mascons' oscillations at tidal frequencies $M_{2}, K_{1}, O_{1}$ and $P_{1}$. In addition to the noise sources listed in section 5.1, residual noise in the first peak may be due to errors in other tides, interannual signals, accelerating mass changes such as those in Greenland, year-to-year fluctuations in the annual amplitudes, mis-modelled short-period atmospheric and oceanic processes, aliased long period non-tidal ocean signals, or signals caused by ocean tides south of $50^{\circ} \mathrm{N}$. The reductions shown in figures $5.7 \mathrm{c}, \mathrm{d}$ increase at frequencies around $0.04 \mathrm{~Hz}$ due to roundoff errors caused by dividing by the spectra in figure 5.7a which approach zero around that frequency. 

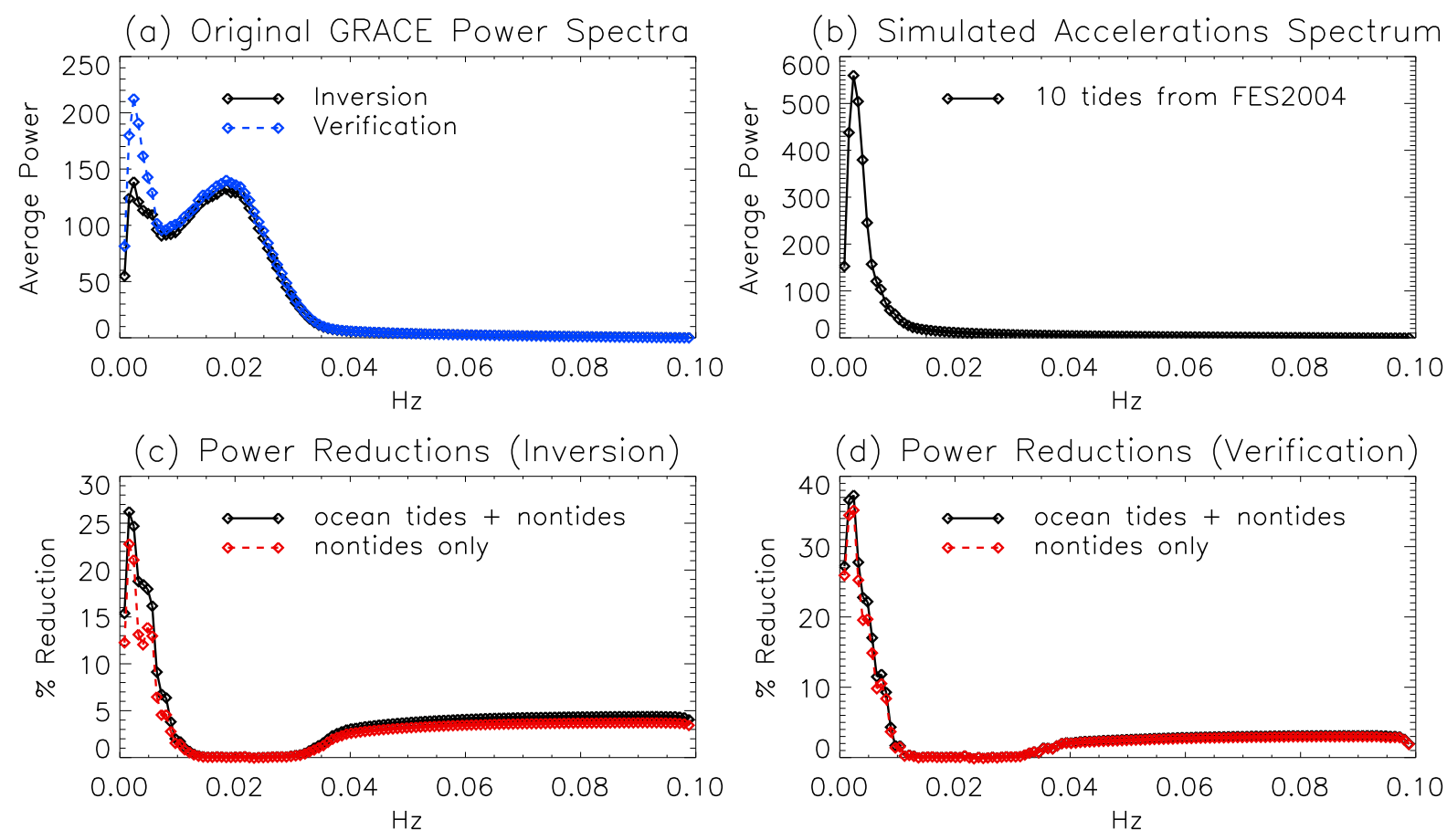

Figure 5.7: (a) GRACE accelerations filtered with a 50 second cutoff period are split into two segments: one (solid black curve) used to invert for mascon estimates and another (dashed blue curve) used only for verification, then sent through a Fast Fourier Transform to obtain power spectra; (b) a synthetic GRACE spectrum using ten tides in FES2004 $\left(M_{2}, K_{1}, K_{2}, O_{1}, P_{1}, S_{2}, N_{2}, Q_{1}, M_{f}, M_{m}\right)$; (c) and (d) show the relative variance reductions due to upward-continued mascon estimates for both inversion and verification accelerations - the solid black curves include ocean tides and nontidal parameters, while the dashed red curves only include nontidal parameters. 


\subsection{The $K_{1}$ North Pole Anomaly}

The $K_{1}$ inversions in figures $4.1 \mathrm{~h}$ (simulated data) and $5.2 \mathrm{f}$ (real data) reveal an anomaly at the north pole which is plotted again in figure 5.8a. The possibility that this anomaly is caused by an interaction between the $K_{1}$ tide and the Tikhonov regularization required by using closely spaced mascons was investigated by performing an inversion of $K_{1}$ using mascons spaced $400 \mathrm{~km}$ apart, as seen in figure 5.8b. This does slightly attenuate the $K_{1}$ north pole anomaly, but also reduces the resolution and could simply be the result of averaging the same anomaly over a larger mascon or the need for a different Tikhonov damping parameter when using $400 \mathrm{~km}$ spaced mascons. The possibility that this anomaly is related to an interaction between the $K_{1}$ tide and the axis of symmetry of the region being inverted was dismissed by inverting for $K_{1}$ using an irregularly-shaped region (see figure 5.8c), which has no significant effect on the $K_{1}$ north pole anomaly. The possibility that this anomaly is related to an interaction between the $K_{1}$ tide and the axis of symmetry of the grid was dismissed by inverting for $K_{1}$ using mascons spaced $230 \mathrm{~km}$ apart but with the axis of symmetry of the grid rotated by $48^{\circ}$ away from the north pole (see figure $5.8 \mathrm{~d}$ ). This also didn't appreciably affect the $K_{1}$ north pole anomaly, so the investigation shifted from possible methodological errors to the geophysical properties of the $K_{1}$ tide's forcing potential.

At latitudes south of the turning latitude $\left(89^{\circ} \mathrm{N}\right)$ GRACE samples the $K_{1}$ tide twice during each tidal period on the ascending/descending passes. Because $K_{1}$ is a diurnal tide, the ascending and descending passes measure phases that are nearly $180^{\circ}$ apart, which prevents aliasing. At the turning latitude, however, the tide is only measured with maximum sensitivity once during each period because GRACE's sensitivity is strongest directly underneath the GRACE midpoint and because the $K_{1}$ forcing potential is stationary in inertial space $^{1}$. If the GRACE orbit didn't precess, it would always sample the same phase of $K_{1}$

\footnotetext{
${ }^{1}$ Richard Ray (personal communication, 2009).
} 
at the turning latitude, yielding an infinitely long alias period. However, the GRACE orbit does slowly precess (Knudsen and Andersen 2002), which results in $K_{1}$ 's finite $\sim 7.5$ year alias period (Ray and Luthcke 2006) in the GRACE data at the turning latitude.

As the data span has lengthened from five to seven years, the $K_{1}$ north pole anomaly has shrunk in amplitude and geographical extent (see inset in figure 5.2f.) In contrast, at all points on the globe $K_{2}$ should display the same behavior that $K_{1}$ does at the pole because $K_{2}$ 's semi-diurnal symmetry means that even at points far from the pole, ascending and descending passes both measure the same phase, which doesn't satisfy the Nyquist-Shannon sampling theorem (Jerri 1977). Inversions of real data (not shown) confirm that $K_{2}$ is unstable over the entire region north of $50^{\circ} \mathrm{N}$ even with seven years of GRACE data.

The FES2004 solution for every tidal constituent except $S_{1}$ has an irregularly shaped region $\sim 40 \mathrm{~km}$ in diameter, centered on the north pole, that is treated as land by the model, in the sense that the tides aren't modeled there. However, this hole is independent of the data span and should affect $M_{2}$ more than $K_{1}$ because $M_{2}$ has a larger amplitude around the hole, so the hole probably isn't contributing significantly to the north pole anomaly in $K_{1}$.

\subsection{Comparisons with Tide Gauge Data}

The FES2004 model is distributed with software that can be used to produce a time series of the sum of all modelled tides at any specified point in the ocean, which we call "autoFES." The autoFES-derived time series is compared to in situ time series from $\sim 30$ tide gauges in the oceans north of $50^{\circ} \mathrm{N}$. An example of these comparisons which uses the tide gauge at Yakutat, AK is shown in table 5.2. The "in situ" column lists the amplitudes and phases of a least-squares fit at many simultaneous tidal frequencies performed on the in situ tide gauge data; the "autoFES" column is the result of an identical analysis applied to the autoFES time series. These time series are then subtracted, and an identical analysis is applied to this differenced time series, which is shown in the "diff" column. Listed at 
(a) K1 Amplitude, $230 \mathrm{~km}$ Grid
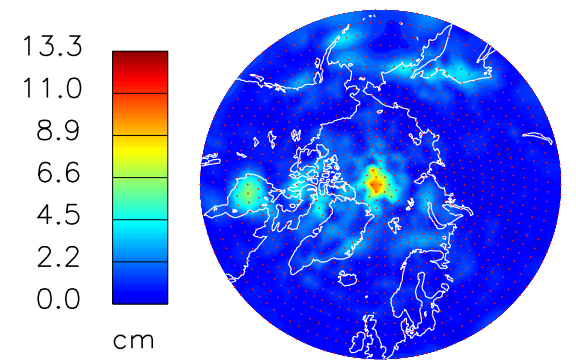

(c) K1 Amplitude, Irregular Region
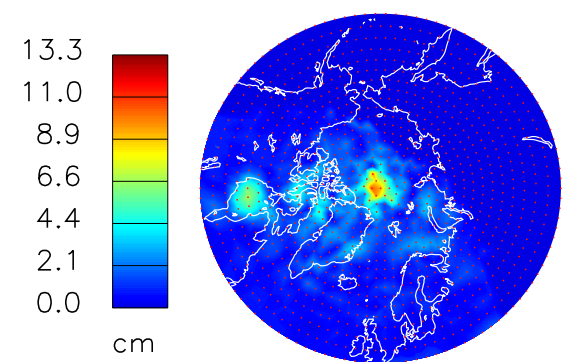

(b) K1 Amplitude, $400 \mathrm{~km}$ Grid
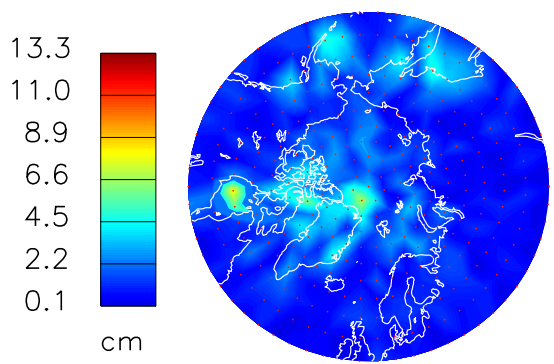

(d) K1 Amplitude, $230 \mathrm{~km}$ Rotated Grid

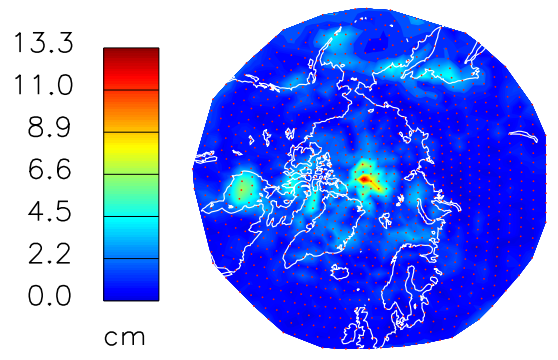

Figure 5.8: Investigation of the $K_{1}$ north pole anomaly; (a) shows $K_{1}$ inverted with mascons spaced $230 \mathrm{~km}$ apart; (b) shows $K_{1}$ inverted with mascons spaced $400 \mathrm{~km}$ apart; (c) shows $K_{1}$ inverted using an irregularly-shaped region; (d) shows $K_{1}$ inverted with mascons spaced $230 \mathrm{~km}$ apart but with the axis of symmetry of the grid rotated by $48^{\circ}$ away from the north pole. 
the top are RMS values for all three of these time series, and a performance metric called "Quality" which is defined as the RMS of the in situ time series divided by the RMS of the differenced time series. A larger quality indicates better agreement between the in situ time series and the autoFES time series. The "Quality" column in the table is defined as the amplitude of the in situ time series at that frequency divided by the amplitude of the differenced time series at the same frequency. Larger qualities indicate better agreement at that row's frequency.

Next, the amplitude and phase maps from FES2004 are averaged over the $230 \mathrm{~km}$ mascons used in the GRACE inversion algorithm. This process results in the FES2004 maps shown in figure 5.2, which have considerably lower spatial resolutions than the $0.0125^{\circ}$ spacings in the original FES2004 maps. These amplitudes and phases are then interpolated to the position of Yakutat, and used in equation (1.29) to obtain a time series of the sum of all modelled tides at Yakutat. This time series is called "masconFES," and it agrees well with autoFES as demonstrated in table 5.3. The only exception is the $S_{1}$ tide which has a quality that's substantially less than one; because the amplitude matches but the phase doesn't, this is likely due to an inconsistency between FES2004 and equations (1.28) and (1.29) with respect to the $S_{1}$ tide. The $S_{1}$ tide has significant radiational forcing in addition to tidal gravitational forcing, which is responsible for a phase shift in FES2004's Greenwich phase lag for the $S_{1}$ tide $^{2}$.

Residual estimates similar to those in figure 5.2 (for tides $M_{2}, K_{1}, O_{1}, P_{1}, N_{2}, K_{2}$ ) are then used to create a time series in the same manner as with masconFES, which is called "real data" in table 5.4. The real data time series is then compared to the time series produced by subtracting autoFES from the in situ data, which is referred to as "insitu-FES." These time series don't agree well, implying that GRACE-derived tidal corrections can't currently improve tide gauge comparisons. This is probably due to the short-scale variability of ocean tides, which strongly affects tide gauge data but can't be resolved by GRACE.

\footnotetext{
${ }^{2}$ Shailen Desai (personal communication, 2009).
} 


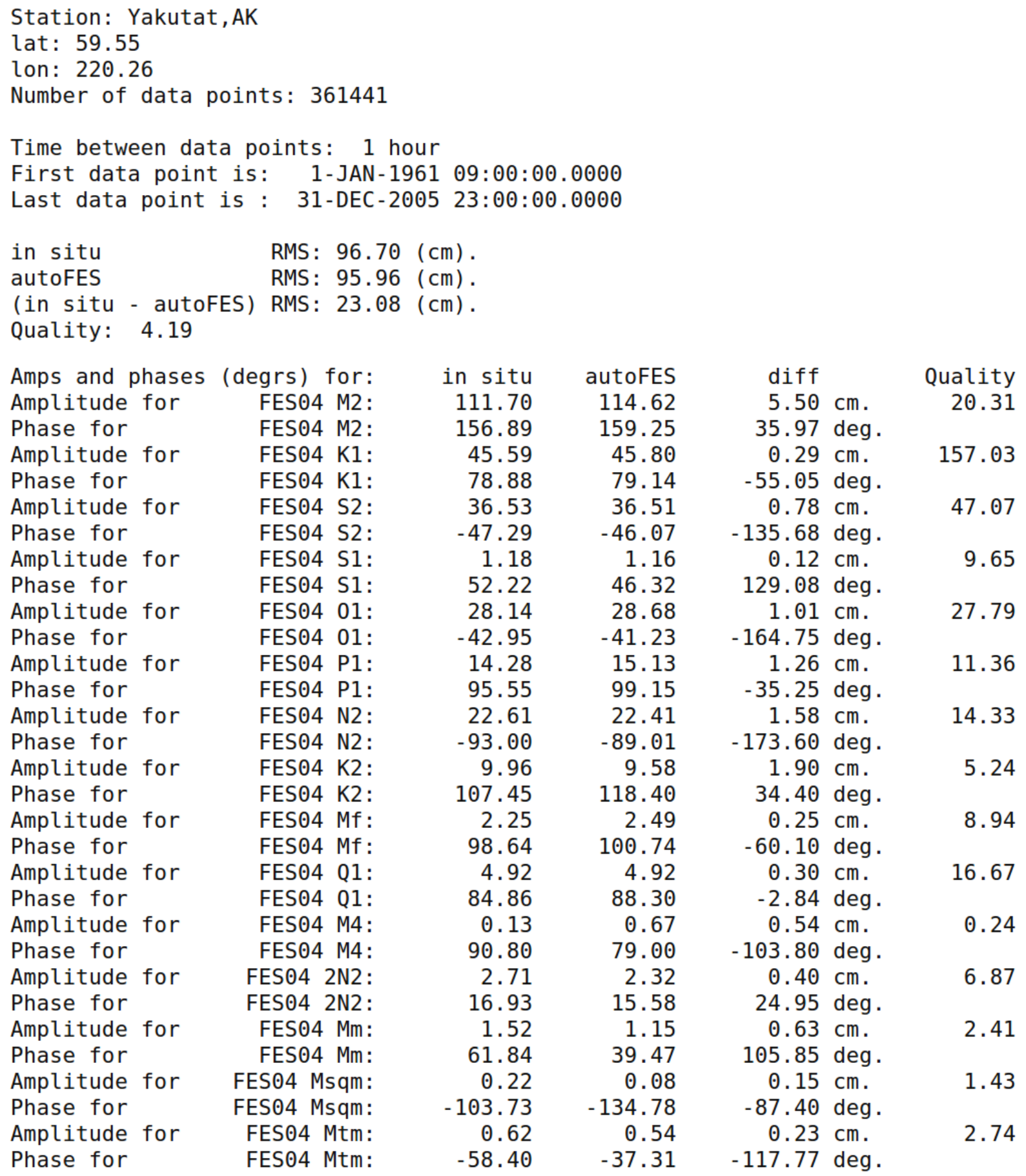

Table 5.2: AutoFES versus in situ tide gauge data at Yakutat, AK 


\begin{tabular}{|c|c|c|c|c|c|c|}
\hline \multicolumn{7}{|c|}{$\begin{array}{l}\text { Station: Yakutat, AK } \\
\text { lat: } 59.548333333 \\
\text { lon: } 220.265000000 \\
\text { Number of data points: } 361441\end{array}$} \\
\hline \multicolumn{7}{|c|}{ 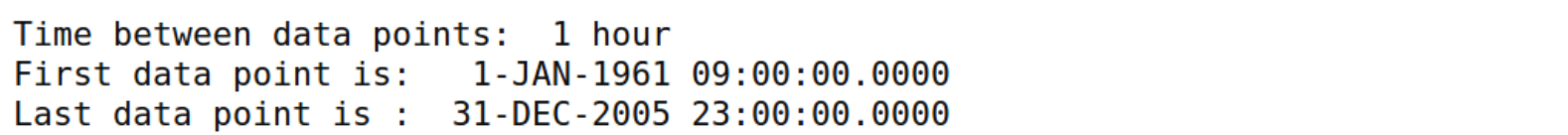 } \\
\hline \multicolumn{7}{|c|}{$\begin{array}{ll}\text { autoFES } & \text { RMS: } 95.96(\mathrm{~cm}) . \\
\text { masconFES } & \text { RMS: } 111.26(\mathrm{~cm}) . \\
\text { (autoFES - masconFES) } & \text { RMS: } 19.27(\mathrm{~cm}) . \\
\text { Quality: } 4.98 & \end{array}$} \\
\hline Amps and phases (degrs) & ) for: & autoFES & masconFES & diff & & Quality \\
\hline Amplitude for & M2: & 114.62 & 132.38 & 19.05 & $\mathrm{~cm}$. & 6.02 \\
\hline Phase for & M2: & 159.25 & 156.05 & -43.58 & deg. & \\
\hline Amplitude for & K1: & 45.80 & 55.17 & 9.80 & $\mathrm{~cm}$. & 4.67 \\
\hline Phase for & K1: & 79.14 & 75.88 & -119.52 & deg. & \\
\hline Amplitude for & S2: & 36.51 & 42.14 & 6.17 & $\mathrm{~cm}$. & 5.92 \\
\hline Phase for & S2: & -46.07 & -49.77 & 107.80 & deg. & \\
\hline Amplitude for & S1: & 1.16 & 1.37 & 2.51 & $\mathrm{~cm}$. & 0.46 \\
\hline Phase for & S1: & 46.32 & -150.42 & 37.24 & deg. & \\
\hline Amplitude for & 01: & 28.68 & 34.49 & 5.82 & $\mathrm{~cm}$. & 4.93 \\
\hline Phase for & 01: & -41.23 & -41.94 & 134.55 & deg. & \\
\hline Amplitude for & P1: & 15.13 & 18.29 & 3.23 & $\mathrm{~cm}$. & 4.69 \\
\hline Phase for & P1: & 99.15 & 96.89 & -93.80 & deg. & \\
\hline Amplitude for & N2: & 22.41 & 26.15 & 4.07 & $\mathrm{~cm}$. & 5.50 \\
\hline Phase for & N2: & -89.01 & -92.84 & 65.64 & deg. & \\
\hline Amplitude for & K2: & 9.58 & 12.39 & 3.02 & $\mathrm{~cm}$. & 3.18 \\
\hline Phase for & K2: & 118.40 & 112.53 & -86.43 & deg. & \\
\hline Amplitude for & Mf: & 2.49 & 2.94 & 0.54 & $\mathrm{~cm}$. & 4.64 \\
\hline Phase for & Mf : & 100.74 & 94.64 & -114.94 & deg. & \\
\hline Amplitude for & Q1: & 4.92 & 5.96 & 1.05 & $\mathrm{~cm}$. & 4.70 \\
\hline Phase for & Q1: & 88.30 & 87.31 & -97.35 & deg. & \\
\hline Amplitude for & M4: & 0.67 & 0.72 & 0.07 & $\mathrm{~cm}$. & 9.18 \\
\hline Phase for & M4: & 79.00 & 74.39 & -153.11 & deg. & \\
\hline Amplitude for & 2N2: & 2.32 & 2.76 & 0.46 & $\mathrm{~cm}$. & 5.05 \\
\hline Phase for & 2N2: & 15.58 & 12.15 & 174.58 & deg. & \\
\hline Amplitude for & $\mathrm{Mm}$ : & 1.15 & 1.40 & 0.24 & $\mathrm{~cm}$. & 4.77 \\
\hline Phase for & $\mathrm{Mm}$ : & 39.47 & 39.07 & -142.87 & deg. & \\
\hline Amplitude for & Msqm: & 0.08 & 0.10 & 0.02 & $\mathrm{~cm}$. & 4.78 \\
\hline Phase for & Msqm: & -134.78 & -143.23 & -7.84 & deg. & \\
\hline Amplitude for & Mtm: & 0.54 & 0.64 & 0.11 & $\mathrm{~cm}$. & 4.88 \\
\hline Phase for & Mtm: & -37.31 & -41.49 & 117.66 & deg. & \\
\hline
\end{tabular}

Table 5.3: MasconFES versus autoFES at Yakutat, AK 
Station: Yakutat, AK

lat: 59.548333333

lon: 220.265000000

Number of data points: 361441

Time between data points: 1 hour

First data point is: 1-JAN-1961 09:00:00.0000

Last data point is : 31-DEC-2005 23:00:00.0000

insitu-FES RMS: $23.08(\mathrm{~cm})$.

real data RMS: $8.67(\mathrm{~cm})$.

(insitu-FES - real data) RMS: $24.93(\mathrm{~cm})$.

Quality: 0.93

\begin{tabular}{|c|c|c|c|c|c|c|}
\hline Amps and phase & (degrs) for: & situ-FES & eal data & diff & & Quality \\
\hline Amplitude for & FESO4 M2: & 5.50 & 4.08 & 8.99 & $\mathrm{~cm}$. & 0.61 \\
\hline Phase for & FESO4 M2: & 35.97 & -103.17 & 53.23 & deg. & \\
\hline Amplitude for & FESO4 K1: & 0.29 & 0.00 & 0.29 & cm. & 1.00 \\
\hline Phase for & FESO4 K1: & -55.05 & -44.81 & -55.05 & deg. & \\
\hline Amplitude for & FESO4 S2: & 0.78 & 0.00 & 0.78 & $\mathrm{~cm}$. & 1.00 \\
\hline Phase for & FESO4 S2: & -135.68 & 131.85 & -135.68 & deg. & \\
\hline Amplitude for & FESO4 S1: & 0.12 & 0.00 & 0.12 & $\mathrm{~cm}$. & 1.00 \\
\hline Phase for & FESO4 S1: & 129.08 & 22.38 & 129.08 & deg. & \\
\hline Amplitude for & FESO4 01: & 1.01 & 5.33 & 4.67 & $\mathrm{~cm}$. & 0.22 \\
\hline Phase for & FESO4 01: & -164.75 & 149.59 & -39.33 & deg. & \\
\hline Amplitude for & FESO4 P1: & 1.26 & 3.51 & 2.86 & $\mathrm{~cm}$. & 0.44 \\
\hline Phase for & FESO4 P1: & -35.25 & 14.10 & -146.43 & deg. & \\
\hline Amplitude for & FESO4 N2: & 1.58 & 1.07 & 1.47 & cm. & 1.07 \\
\hline Phase for & FESO4 N2: & -173.60 & 122.23 & -132.57 & deg. & \\
\hline Amplitude for & FESO4 K2: & 1.90 & 9.58 & 10.19 & cm. & 0.19 \\
\hline Phase for & FESO4 K2: & 34.40 & -69.06 & 100.48 & deg. & \\
\hline Amplitude for & FESO4 Mf: & 0.25 & 0.00 & 0.25 & $\mathrm{~cm}$. & 1.00 \\
\hline Phase for & FESO4 Mf: & -60.10 & 46.90 & -60.10 & deg. & \\
\hline Amplitude for & FESO4 Q1: & 0.30 & 0.00 & 0.30 & $\mathrm{~cm}$. & 1.00 \\
\hline Phase for & FESO4 Q1: & -2.84 & -57.69 & -2.84 & deg. & \\
\hline Amplitude for & FESO4 M4: & 0.54 & 0.00 & 0.54 & $\mathrm{~cm}$. & 1.00 \\
\hline Phase for & FESO4 M4: & -103.80 & -169.47 & -103.80 & deg. & \\
\hline Amplitude for & FESO4 2N2: & 0.40 & 0.00 & 0.40 & $\mathrm{~cm}$. & 1.00 \\
\hline Phase for & FESO4 2N2: & 24.95 & -176.15 & 24.95 & deg. & \\
\hline Amplitude for & FES๑4 Mm: & 0.63 & 0.00 & 0.63 & $\mathrm{~cm}$. & 1.00 \\
\hline Phase for & FESO4 Mm: & 105.85 & -39.34 & 105.85 & deg. & \\
\hline Amplitude for & FESO4 Msqm: & 0.15 & 0.00 & 0.15 & $\mathrm{~cm}$. & 1.00 \\
\hline Phase for & FESO4 Msqm: & -87.40 & -53.26 & -87.40 & deg. & \\
\hline Amplitude for & FESO4 Mtm: & 0.23 & 0.00 & 0.23 & $\mathrm{~cm}$ & 1.00 \\
\hline Phase for & FESO4 Mtm: & -117.77 & 9.24 & -117.77 & deg. & \\
\hline
\end{tabular}

Table 5.4: Inverted real data versus in situ tide gauge minus autoFES at Yakutat, AK 


\section{Chapter 6}

\section{Conclusions}

We have used seven years of GRACE acceleration data to solve for $M_{2}, K_{1}, K_{2}, O_{1}, P_{1}$, $Q_{1}, N_{2}, M_{f}$, and $M_{m}$. The solutions look plausible, except for $K_{1}$ near the north pole and $K_{2}$ in at least the entire region north of $50^{\circ} \mathrm{N}$, limitations which are likely due to aliasing. Ocean tides reduce the variance of accelerations not used by the inversion nearly as well as they reduce the variance of accelerations used in the inversion. This suggests that the ocean mascon tidal solutions can improve GRACE processing. The variance reduction due to land tides drops off more sharply when applied to accelerations not used in the inversion. The land tides are used as uncertainty estimates which confirm the $\sim 1 \mathrm{~cm}$ uncertainty derived by subtracting two independent solutions based on 3.5 years of data. Solutions with improved accuracies might be obtained by inverting GRACE data using code which simultaneously solves for GRACE's orbital parameters as well as surface mascons, such as JPL's MIRAGE software.

Truncation errors, land mask errors and errors in the assumed density of seawater are too small to account for residual features that rise above the noise floor. These features are probably caused by errors in the FES2004 amplitudes and phases. For instance, seasonal ice cover in Hudson Bay hinders altimeters and could contribute to the $K_{1}$ residual feature there because FES2004 uses ERS crossover data in Hudson Bay, and the alias period of $K_{1}$ in the ERS data is 365.25 days (Ray et al. 2003). Adding the residual estimates to FES2004 indicates that FES2004 overestimates the $K_{1}$ amplitude by up to $50 \%$ in Hudson Bay and 
north of Siberia. In fact, the signs of the residual estimates suggest that FES2004 amplitudes for $M_{2}$ and $K_{1}$, as well as for $O_{1}$ and $P_{1}$ (not shown), tend to be too large in many regions throughout the oceans north of $50^{\circ} \mathrm{N}$.

All large $M_{2}$ residual features are spatially correlated with large amplitudes in the original FES2004 $M_{2}$ model, such as those in Hudson Strait, southeast of Greenland, northeast of Scandinavia, off the coast of Alaska, and in the Sea of Okhotsk. Similarly, most large $K_{1}$ residual features are spatially correlated with large amplitudes in the original FES2004 $K_{1}$ model, such as those in Hudson and Baffin Bays, the Canadian archipelago, northeast of Scandinavia, and in the Sea of Okhotsk. The only exception is the $\sim 3 \mathrm{~cm} K_{1}$ residual feature in Hudson Strait, where FES2004 shows a $K_{1}$ amplitude of only $\sim 2 \mathrm{~cm}$. This correlation suggests errors in FES2004 are proportional to tidal amplitude, which wouldn't be the case for a tide model based purely on altimetry data.

Large features in the residual tidal estimates could be associated with poor coverage of tide gauge observations assimilated by FES, or large errors in nearby tide gauges. Flaws in the bathymetry model used by FES2004 might also play a role, especially in shallow waters like Hudson Strait. The residual estimates aren't larger north of the TOPEX/Poseidon turning latitude of $66^{\circ} \mathrm{N}$. FES2004 may be overcoming this lack of coverage with its hydrodynamic modelling and its assimilation of Arctic tide gauge data and crossover points from the ERS satellite which has an $\sim 81^{\circ} \mathrm{N}$ turning latitude.

Future work will be necessary to use the GRACE-derived tidal corrections to improve tide gauge predictions, likely because point measurements from tide gauges are more sensitive to local variations in tidal amplitudes and phases than GRACE. In the meantime, the residual estimates are being added to FES2004 to produce a new tide model which should improve GRACE processing and any other application that doesn't strongly depend on short-scale effects.

Also, the inversion algorithm is being extended to solve for mascon time dependences that aren't simple polynomials or harmonic functions of time. Instead, the mascons' time 
dependencies are being generalized so they can represent mass changes as arbitrarily-defined functions of time. The inversion algorithm will then solve for scale factors multiplying these arbitrary functions of time at each mascon. Once complete, this project may help reveal the geographic extent of a recently-discovered (Peralta Ferriz et al. 2010) mode of variability of ocean bottom pressure in the Arctic ocean. 


\section{Bibliography}

Aster, R., Thurber, C., and Borchers, B. (2005). Parameter estimation and inverse problems. Academic Press.

Barmin, M., Ritzwoller, M., and Levshin, A. (2001). A fast and reliable method for surface wave tomography. Pure and Applied Geophysics, 158(8):1351-1375.

Cartwright, D. and Edden, A. (1973). Corrected tables of tidal harmonics. Geophys. JR astr. Soc, 33(3):253-264.

Cartwright, D. and Tayler, R. (1971). New Computations of the Tide-generating Potential. Geophysical Journal of the Royal Astronomical Society, 23(1):45-73.

Case, K., Kruizinga, G., and Wu, S. (2002). GRACE Level 1B Data Product User Handbook. JPL Publication D-22027.

Dahlen, R. (1993). Effect of the Earth's ellipticity on the lunar tidal potential. Geophysical Journal International, 113(1):250-251.

Darwin, G. (1883). Reports of a committee for the harmonic analysis of tides. British Association for the Advancement of Science.

Desai, S. (1996). Ocean Tides from TOPEX/POSEIDON altimetry with some geophysical applications. Ph.D. thesis, University of Colorado.

Desai, S. and Yuan, D. (2006). Application of the convolution formation to the ocean tide potential: results from the Gravity and Recovery and Climate Experiment (GRACE). Journal of Geophysical Research, 111:C06023.

Dickey, J., Bender, P., Faller, J., Newhall, X., Ricklefs, R., Ries, J., Shelus, P., Veillet, C., Whipple, A., Wiant, J., et al. (1994). Lunar laser ranging: A continuing legacy of the Apollo program. Science, 265(5171):482.

Doodson, A. (1921). The harmonic development of the tide-generating potential. Proceedings of the Royal Society of London. Series A, Containing Papers of a Mathematical and Physical Character, 100(704):305-329. 
Egbert, G., Erofeeva, S., Han, S., Luthcke, S., and Ray, R. (2009). Assimilation of GRACE tide solutions into a numerical hydrodynamic inverse model. Geophysical Research Letters, 36(20):L20609.

Farrell, W. (1972). Deformation of the Earth by surface loads. Reviews of Geophysics, 10(3):761-797.

Flechtner, F. (2003). AOD1B product description document. GRACE project documentation, JPL, pages 327-750.

Han, S., Shum, C., and Matsumoto, K. (2005). GRACE observations of M2 and S2 ocean tides underneath the Filchner-Ronne and Larsen ice shelves, Antarctica. Geophysical Research Letters, 32(20):L20311.

Hartmann, T. and Wenzel, H. (1995). The HW95 tidal potential catalogue. Geophysical research letters, $22(24): 3553-3556$.

Jackson, J. (1998). Classical Electrodynamics, 3rd ed. John Wiley \& Sons.

Jerri, A. (1977). The Shannon sampling theoremIts various extensions and applications: A tutorial review. Proceedings of the IEEE, 65(11):1565-1596.

Khan, S., Wahr, J., Bevis, M., Velicogna, I., and Kendrick, E. (2010). Spread of ice mass loss into northwest Greenland observed by GRACE and GPS. Geophysical Research Letters, 37(6):L06501.

Knudsen, P. and Andersen, O. (2002). Correcting GRACE gravity fields for ocean tide effects. Geophysical Research Letters, 29(8):1178.

Kudryavtsev, S. (2004). Improved harmonic development of the Earth tide-generating potential. Journal of Geodesy, 77(12):829-838.

Lambeck, K. (1975). Effects of tidal dissipation in the oceans on the Moon's orbit and the Earth's rotation. Journal of Geophysical Research, 80(20):2917-2925.

Lambeck, K. (1988). Geophysical geodesy. Clarendon Press.

Luthcke, S., Arendt, A., Rowlands, D., McCarthy, J., and Larsen, C. (2008). Recent glacier mass changes in the Gulf of Alaska region from GRACE mascon solutions. Journal of Glaciology, 54(188):767-777.

Lyard, F., Lefevre, F., Letellier, T., and Francis, O. (2006). Modelling the global ocean tides: modern insights from FES2004. Ocean Dynamics, 56(5):394-415.

McCarthy, D. and Petit, G. (2004). IERS conventions (2003). IERS technical note, 32:389888.

Meister, R. and Wingham, D. (2010). Deriving Antarctic Postglacial Rebound rates from GRACE and altimetry. In AGU Fall Meeting Abstracts, volume 1, page 0495. 
Melachroinos, S., Lemoine, J., Tregoning, P., and Biancale, R. (2009). Quantifying FES2004 S 2 tidal model from multiple space-geodesy techniques, GPS and GRACE, over North West Australia. Journal of Geodesy, 83(10):915-923.

Melchior, P. (1983). The tides of the planet Earth. Oxford, Pergamon Press, 1983. 653 p., 1.

Moore, P. and King, M. (2008). Antarctic ice mass balance estimates from GRACE: Tidal aliasing effects. Journal of Geophysical Research-Earth Surface, 113(F2):F02005.

Munk, W. (1997). Once again: once again-tidal friction. Progress in Oceanography, 40(1$4): 7-35$.

Munk, W. and Cartwright, D. (1966). Tidal spectroscopy and prediction. Philosophical Transactions of the Royal Society of London. Series A, Mathematical and Physical Sciences, 259(1105):533-581.

Munk, W. and Macdonald, G. (1975). The rotation of the earth: a geophysical discussion.

Munk, W. and Wunsch, C. (1998). Abyssal recipes II: energetics of tidal and wind mixing. Deep-Sea Research Part I, 45(12):1977-2010.

Peralta Ferriz, A., Morison, J., Wallace, J., Zhang, J., Bonin, J., and Chambers, D. (2010). A new mode of high frequency variability in Arctic Ocean bottom pressure and its possible effects on GRACE solutions. In AGU Fall Meeting Abstracts, volume 1, page 0678.

Platzman, G. (1971). Ocean tides and related waves. Mathematical problems in the geophysical sciences, 1:239-291.

Press, W., Teukolsky, S., Vetterling, W., and Flannery, B. (1992). Numerical recipes in C. Cambridge Univ. Press Cambridge MA, USA:.

Proudman, J. (1960). The Condition that a Long-Period Tide shall follow the EquilibriumLaw. Geophysical Journal of the Royal Astronomical Society, 3(2):244-249.

Ray, R. and Luthcke, S. (2006). Tide model errors and GRACE gravimetry: towards a more realistic assessment. Geophysical Journal International, 167(3):1055-1059.

Ray, R., Luthcke, S., and Boy, J. (2009). Qualitative comparisons of global ocean tide models by analysis of intersatellite ranging data. Journal of Geophysical Research-Oceans, 114(C9):n E AC09017.

Ray, R., Rowlands, D., and Egbert, G. (2003). Tidal models in a new era of satellite gravimetry. Space Science Reviews, 108(1):271-282.

Scharroo, R. (2006). Fixing the S1 Wave of FES2004. Altimetrics LLC.

Schwiderski, E. (1980). On charting global ocean tides. Reviews of Geophysics, 18(1):243268. 
Simon, J., Bretagnon, P., Chapront, J., Chapront-Touzé, M., Francou, G., and Laskar, J. (1994). Numerical expressions for precession formulae and mean elements for the Moon and the planets. Astronomy and Astrophysics, 282:663-683.

Steele, M. and Ermold, W. (2007). Steric sea level change in the Northern Seas. Journal of Climate, 20(3):403-417.

Stephenson, F., Morrison, L., and Whitrow, G. (1984). Long-Term Changes in the Rotation of the Earth: 700 BC to AD 1980 [and Discussion]. Philosophical Transactions of the Royal Society of London. Series A, Mathematical and Physical Sciences, 313(1524):47.

Swenson, S., Chambers, D., and Wahr, J. (2008). Estimating geocenter variations from a combination of GRACE and ocean model output. Journal of Geophysical Research, 113(B8):B08410.

Tapley, B., Bettadpur, S., Watkins, M., and Reigber, C. (2004a). The gravity recovery and climate experiment: Mission overview and early results. Geophysical Research Letters, 31(9):L09607.

Tapley, B., Schutz, B., and Born, G. (2004b). Statistical orbit determination. Academic Press.

Turing, A. M. (1948). Rounding-off Errors in Matrix Processes. The Quarterly Journal of Mechanics and Applied Mathematics, 1(1):287-308.

Wahr, J. (1981). Body tides on an elliptical, rotating, elastic and oceanless Earth. Geophys. JR Astr. Soc, 64:677-703.

Wahr, J. (2007). Time Variable Gravity from Satellites. Treatise on Geophysics, 3:213-237.

Wang, C., Dong, S., and Munoz, E. (2010). Seawater density variations in the North Atlantic and the Atlantic meridional overturning circulation. Climate Dynamics, 34(7):953-968.

Willis, J., Chambers, D., and Nerem, R. (2008). Assessing the globally averaged sea level budget on seasonal to interannual timescales. Journal of Geophysical Research, 113(C6):C06015.

Wu, S., Kruizinga, G., and Bertiger, W. (2006). Algorithm Theoretical Basis Document for GRACE Level-1B Data Processing v1.2. JPL Publication D-27672. 


\section{Appendix A}

\section{Spherical Harmonics}

To solve Laplace's equation in spherical coordinates (Jackson 1998)

$$
\nabla^{2} V(r, \theta, \phi)=0=\frac{1}{r^{2}} \frac{\partial}{\partial r}\left(r^{2} \frac{\partial V}{\partial r}\right)+\frac{1}{r^{2} \sin \theta} \frac{\partial}{\partial \theta}\left(\sin \theta \frac{\partial V}{\partial \theta}\right)+\frac{1}{r^{2} \sin ^{2} \theta} \frac{\partial^{2} V}{\partial \phi^{2}}
$$

assume a separable solution exists in the form $V(r, \theta, \phi)=R(r) T(\theta) L(\phi)$ and use this definition in equation (A.1) to obtain

$$
\frac{1}{R} \frac{\partial}{\partial r}\left(r^{2} \frac{\partial R}{\partial r}\right)=-\left[\frac{1}{T \sin \theta} \frac{\partial}{\partial \theta}\left(\sin \theta \frac{\partial T}{\partial \theta}\right)+\frac{1}{L \sin ^{2} \theta} \frac{\partial^{2} L}{\partial^{2} \phi}\right]
$$

Note that the left hand side (LHS) depends only on $r$, while the RHS depends only on $\theta$ and $\phi$. Since these two expressions could vary independently, their equality is only guaranteed if each is equal to the same constant. With malice of forethought, call this (possibly complex) constant $l(l+1)$ and rewrite equation (A.2) as

$$
\begin{gathered}
\frac{1}{R} \frac{\partial}{\partial r}\left(r^{2} \frac{\partial R}{\partial r}\right)=l(l+1) \\
\frac{1}{T \sin \theta} \frac{\partial}{\partial \theta}\left(\sin \theta \frac{\partial T}{\partial \theta}\right)+\frac{1}{L \sin ^{2} \theta} \frac{\partial^{2} L}{\partial^{2} \phi}=-l(l+1) .
\end{gathered}
$$

Equation (A.3) is satisfied by 


$$
\mathrm{R}(\mathrm{r})=\left\{\begin{array}{l}
r^{l} \\
r^{-(l+1)} .
\end{array}\right.
$$

Meanwhile, multiply equation (A.4) by $\sin ^{2} \theta$ to separate $\theta$ and $\phi$

$$
\frac{\sin \theta}{T} \frac{\partial}{\partial \theta}\left(\sin \theta \frac{\partial T}{\partial \theta}\right)+l(l+1) \sin ^{2} \theta=\frac{-1}{L} \frac{\partial^{2} L}{\partial^{2} \phi}
$$

Again, the independent LHS and RHS are both equal to another constant $m^{2}$.

$$
\begin{gathered}
\frac{-1}{L} \frac{\partial^{2} L}{\partial^{2} \phi}=m^{2} \\
\frac{\sin \theta}{T} \frac{\partial}{\partial \theta}\left(\sin \theta \frac{\partial T}{\partial \theta}\right)+l(l+1) \sin ^{2} \theta=m^{2}
\end{gathered}
$$

Equation (A.6) is satisfied by

$$
\mathrm{L}(\phi)=\left\{\begin{array}{l}
e^{i m \phi} \\
e^{-i m \phi}
\end{array}\right.
$$

Since continuity of $\mathrm{L}$ requires that $L(2 \pi)=L(0), m$ has to be an integer. Finally, rewrite equation (A.7) using the definition $x \equiv \cos \theta$

$$
\frac{\partial}{\partial x}\left[\left(1-x^{2}\right) \frac{\partial T}{\partial x}\right]+\left[l(l+1)-\frac{m^{2}}{1-x^{2}}\right] T=0 .
$$

This is the general Legendre equation, which can be solved by a power series expansion in $x$ 


$$
T(x)=\left(1-x^{2}\right)^{m / 2} \sum_{l=0}^{\infty} a_{l} x^{l} .
$$

Since $\theta$ is defined on $[0, \pi], T(x)$ needs to be well behaved on $[-1,1]$ which only occurs if $l$ is a non-negative integer and $m$ is an integer obeying $|m| \leq l$. For $m=0$, the solutions are known as Legendre polynomials

$$
P_{l}(x)=\frac{1}{2^{l} l !} \frac{\partial^{l}}{\partial x^{l}}\left(x^{2}-1\right)^{l} .
$$

For $m \neq 0$, the solutions are called associated Legendre functions and are defined such that $P_{l}^{0}(x) \equiv P_{l}(x):$

$$
P_{l}^{m}(x)=(-1)^{m}\left(1-x^{2}\right)^{m / 2} \frac{\partial^{m}}{\partial x^{m}} P_{l}(x) .
$$

Here are several useful recursion relations:

$$
\begin{gathered}
(2 l+1) x P_{l}^{m}(x)=(l+1-m) P_{l+1}^{m}(x)+(l+m) P_{l-1}^{m}(x) \\
\left(1-x^{2}\right) \frac{\partial}{\partial x} P_{l}^{m}(x)=(l+m) P_{l-1}^{m}(x)-l x P_{l}^{m}(x) .
\end{gathered}
$$

For fixed $m$, the associated Legendre functions are orthogonal over the region $x \in[-1,1]$ :

$$
\int_{-1}^{1} d x P_{l}^{m}(x) P_{l^{\prime}}^{m}(x)=\frac{2}{(2 l+1)} \frac{(l+m) !}{(l-m) !} \delta_{l l^{\prime}} .
$$

The angular factors are collectively called spherical harmonics

$$
Y_{l}^{m}(\theta, \phi)=\sqrt{\frac{(2 l+1)(l-m) !}{4 \pi(l+m) !}} P_{l}^{m}(\cos \theta) e^{i m \phi}
$$


Table A.1: Associated Legendre functions.

\begin{tabular}{c|c|c|c|c|c} 
& $\mathbf{m}=-\mathbf{2}$ & $\mathbf{m}=-\mathbf{1}$ & $\mathbf{m}=\mathbf{0}$ & $\mathbf{m}=+\mathbf{1}$ & $\mathbf{m}=+\mathbf{2}$ \\
\hline $\mathbf{l}=\mathbf{0}$ & & & 1 & & \\
\hline $\mathbf{l}=\mathbf{1}$ & & $\frac{1}{2} \sqrt{1-x^{2}}$ & $x$ & $-\sqrt{1-x^{2}}$ & \\
\hline $\mathbf{l}=\mathbf{2}$ & $\frac{1}{8}\left(1-x^{2}\right)$ & $\frac{x}{2} \sqrt{1-x^{2}}$ & $\frac{1}{2}\left(3 x^{2}-1\right)$ & $-3 x \sqrt{1-x^{2}}$ & $3\left(1-x^{2}\right)$ \\
\hline
\end{tabular}

which have the following properties under parity and coordinate inversion

$$
\begin{gathered}
Y_{l}^{m}(\theta, \phi)=(-1)^{m} Y_{l}^{m *}(\theta, \phi) \\
Y_{l}^{m}(\pi-\theta, \phi+\pi)=(-1)^{l} Y_{l}^{m}(\theta, \phi) .
\end{gathered}
$$

The normalization insures that

$$
\int_{0}^{2 \pi} d \phi \int_{0}^{\pi} \sin \theta d \theta\left[Y_{l}^{m}(\theta, \phi) Y_{l}^{m *}(\theta, \phi)\right]=1
$$

where $Y_{l}^{m *}$ is the complex conjugate of $Y_{l}^{m}$. They're also orthogonal over the unit sphere

$$
\int_{0}^{2 \pi} d \phi \int_{0}^{\pi} \sin \theta d \theta\left[Y_{l}^{m}(\theta, \phi) Y_{l^{\prime}}^{m^{\prime} *}(\theta, \phi)\right]=\delta_{l l^{\prime}} \delta_{m m^{\prime}}
$$

The spherical harmonics form a complete basis set over the unit sphere, in the sense that any sufficiently smooth, (potentially) complex function $V_{c}(\theta, \phi)$ defined on the unit sphere has values $A_{l}^{m}$ satisfying

$$
V_{c}(\theta, \phi)=\sum_{l=0}^{\infty} \sum_{m=-l}^{l} A_{l}^{m} Y_{l}^{m}(\theta, \phi)
$$


where the values $A_{l}^{m}$ can be determined by multiplying equation (A.20) by $Y_{l^{\prime}}^{m^{\prime} *}$ and integrating over the unit sphere. The orthogonality of the $Y_{l}^{m}$ 's gives:

$$
A_{l}^{m}=\int_{0}^{2 \pi} d \phi \int_{0}^{\pi} \sin \theta d \theta V_{c}(\theta, \phi) Y_{l}^{m *}(\theta, \phi)
$$

For example, a commonly expanded function is the gravitational potential due to a point mass

$$
\frac{1}{\left|\overrightarrow{\mathbf{r}^{\prime}}-\overrightarrow{\mathbf{r}}\right|}=4 \pi \sum_{l=0}^{\infty}\left(\frac{1}{2 l+1}\right) \frac{r_{<}^{l}}{r_{>}^{l+1}} \sum_{m=-l}^{l} Y_{l}^{m *}\left(\theta^{\prime}, \phi^{\prime}\right) Y_{l}^{m}(\theta, \phi)
$$

The function to be expanded is often real, which allows $m$ to range from 0 to $l$ by using equation (A.16) to combine $Y_{l}^{m}$ 's with equal values of $|m|$.

$$
\begin{aligned}
Y_{l}^{0} & =\text { real } \\
Y_{l}^{m *}\left(\theta^{\prime}, \phi^{\prime}\right) Y_{l}^{m}(\theta, \phi)+Y_{l}^{-m *}\left(\theta^{\prime}, \phi^{\prime}\right) Y_{l}^{-m}(\theta, \phi) & =2 \operatorname{Re}\left[Y_{l}^{m *}\left(\theta^{\prime}, \phi^{\prime}\right) Y_{l}^{m}(\theta, \phi)\right]
\end{aligned}
$$

Any sufficiently smooth real function $V_{r}(\theta, \phi)$ can thus be expanded as

$$
V_{r}(\theta, \phi)=\operatorname{Re}\left[\sum_{l=0}^{\infty} \sum_{m=0}^{l}\left(2-\delta_{m 0}\right) A_{l}^{m} Y_{l}^{m}(\theta, \phi)\right]
$$

which is more commonly written as

$$
V_{r}(\theta, \phi)=\sum_{l=0}^{\infty} \sum_{m=0}^{l} P_{l}^{m}(\cos \theta)\left[C_{l}^{m} \cos (m \phi)+S_{l}^{m} \sin (m \phi)\right]
$$

where the dimensionless Stokes coefficients $C_{l}^{m}$ and $S_{l}^{m}$ can be determined using 
Table A.2: Spherical harmonics $Y_{l}^{m}(\theta, \phi)$ for $l=0,1,2$.

\begin{tabular}{l|c|c|c} 
& $\mathbf{m}=\mathbf{0}$ & $\mathbf{m}= \pm \mathbf{1}$ & $\mathbf{m}= \pm \mathbf{2}$ \\
\hline $\mathbf{l}=\mathbf{0}$ & $\sqrt{\frac{1}{4 \pi}}$ & & \\
\hline $\mathbf{l}=\mathbf{1}$ & $\sqrt{\frac{3}{4 \pi}} \cos \theta$ & $\mp \sqrt{\frac{3}{8 \pi}} \sin \theta e^{ \pm i \phi}$ & \\
\hline $\mathbf{l}=\mathbf{2}$ & $\sqrt{\frac{5}{16 \pi}}\left(3 \cos ^{2} \theta-1\right)$ & $\mp \sqrt{\frac{15}{8 \pi}} \sin \theta \cos \theta e^{ \pm i \phi}$ & $\sqrt{\frac{15}{32 \pi}} \sin ^{2} \theta e^{ \pm 2 i \phi}$ \\
\hline
\end{tabular}

$$
\begin{aligned}
& C_{l}^{m}=\left(2-\delta_{m 0}\right) \frac{(2 l+1)(l-m) !}{4 \pi(l+m) !} \int_{0}^{2 \pi} d \phi \int_{0}^{\pi} \sin \theta d \theta V_{r}(\theta, \phi) P_{l}^{m}(\theta, \phi) \cos (m \phi) \\
& S_{l}^{m}=\left(2-\delta_{m 0}\right) \frac{(2 l+1)(l-m) !}{4 \pi(l+m) !} \int_{0}^{2 \pi} d \phi \int_{0}^{\pi} \sin \theta d \theta V_{r}(\theta, \phi) P_{l}^{m}(\theta, \phi) \sin (m \phi) .
\end{aligned}
$$

A useful relation between spherical harmonics and Legendre polynomials is the addition theorem

$$
P_{l}(\cos \gamma)=\frac{4 \pi}{2 l+1} \sum_{m=-l}^{l} Y_{l}^{m *}\left(\theta^{\prime}, \phi^{\prime}\right) Y_{l}^{m}(\theta, \phi)
$$

where $\gamma$ is the angle between the points $(\theta, \phi)$ and $\left(\theta^{\prime}, \phi^{\prime}\right)$ on the unit sphere

$$
\cos \gamma=\cos \theta \cos \theta^{\prime}+\sin \theta \sin \theta^{\prime} \cos \left(\phi-\phi^{\prime}\right)
$$

Each $Y_{l}^{m}$ has a spatial wavelength determined by its degree $l$ and order $m$ which can be calculated on a spherical Earth. For $m=0$, the $Y_{l}^{0}$ functions are polynomials of degree $\cos ^{l} \theta$. These functions have $l$ zeros in the $\sim 20,000 \mathrm{~km}$ from the north to south pole, which implies that $Y_{l}^{0}$ wavelengths are $\frac{40,000}{l} \mathrm{~km}$, and that they're oriented north-south. For $m \neq 0$, the $Y_{l}^{m}$ 's depend on $\phi$ through $e^{i m \phi}$, which have $2 m$ zeros in the $\sim 40,000 \mathrm{~km}$ around the equator, which implies that $Y_{l}^{m}$ functions have east-west wavelengths of $\frac{40,000}{m} \mathrm{~km}$. 
For some applications, the half-wavelength is more relevant: each $Y_{l}^{m}$ has a halfwavelength of $\frac{20,000}{l} \mathrm{~km}$ which is oriented north-south if $m=0$ and east-west if $m=l$. 


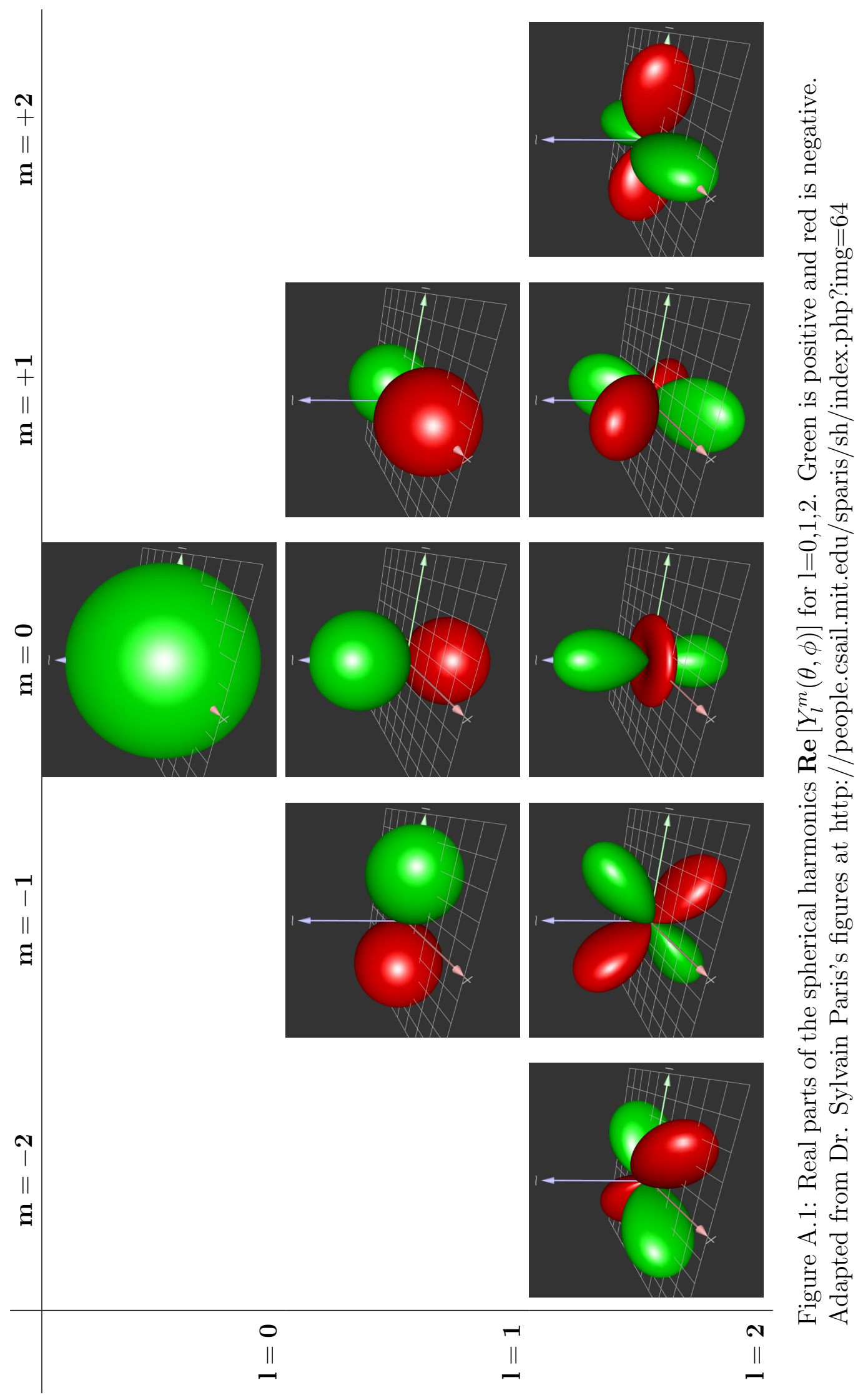

